Portland State University

PDXScholar

\title{
Supervisor-Subordinate Directional Age Differences and Employee Reactions to Formal Performance Feedback: Examining Mediating and Moderating Mechanisms in a Chinese Sample
}

Gabriela Burlacu

Portland State University

Follow this and additional works at: https://pdxscholar.library.pdx.edu/open_access_etds

Part of the Industrial and Organizational Psychology Commons, and the Work, Economy and Organizations Commons Let us know how access to this document benefits you.

\section{Recommended Citation}

Burlacu, Gabriela, "Supervisor-Subordinate Directional Age Differences and Employee Reactions to Formal Performance Feedback: Examining Mediating and Moderating Mechanisms in a Chinese Sample" (2013). Dissertations and Theses. Paper 662.

https://doi.org/10.15760/etd.662

This Dissertation is brought to you for free and open access. It has been accepted for inclusion in Dissertations and Theses by an authorized administrator of PDXScholar. Please contact us if we can make this document more accessible: pdxscholar@pdx.edu. 
Supervisor-Subordinate Directional Age Differences and Employee Reactions to Formal Performance Feedback:

Examining Mediating and Moderating Mechanisms in a Chinese Sample

by

Gabriela Burlacu

A dissertation submitted in partial fulfillment of the requirements for the degree of

Doctor of Philosophy

in

Applied Psychology

Dissertation Committee:

Keith James, Chair

Donald Truxillo

Todd Bodner

Mo Wang

DeLys Ostlund

Portland State University

2013 


\begin{abstract}
As a result of changing demographic trends in today's workforce, employees of all ages can now be found in all career stages. Consequently, the pairing of a younger supervisor with a relatively older employee is becoming increasingly more common. Research in the United States has shown that such demographically "non-normative" pairings have negative implications for employee attitudes and behaviors, and thus for employee performance management. However, little is known about the effects of such pairings in other nations and cultures, despite the fact that these demographic shifts are occurring on a global level.

As such, this study examined the effects of these pairings on employee reactions to formal performance feedback episodes in a large organization in China, due to the nation's similarly shifting demographic trends and its economic power in today's global economy. A series of path analyses showed that being paired with a relatively younger supervisor did predict reduced employee feedback satisfaction and perceptions of feedback utility; but, contrary to the proposed model, these effects did not occur because of reduced interactional justice perceptions or reduced perceptions of leader-member exchange (LMX). Further, LMX did not moderate the study outcomes, demonstrating that having a
\end{abstract}


better relationship with one's supervisor did not alleviate the effects of supervisor relative age on employee feedback reactions.

The conceptual and practical implications of these results are discussed in light of a rapidly changing workforce, and of cultural differences, in China. 


\section{Dedication}

This dissertation is dedicated to the next generation of workers, whose work experiences of tomorrow will depend largely on the decisions we make today. I also dedicate this dissertation to one little worker bee in particular: my endlessly intelligent and curious nephew, Anderson George Cosmas, who gives me good reason to try to make the world a better place. 


\section{Acknowledgments}

I would like to acknowledge my dissertation committee, Drs. Keith James, Todd Bodner, DeLys Ostlund, Donald Truxillo, and Mo Wang, for their guidance with this project and their unyielding support throughout my graduate school experience. I would also like to acknowledge Jenna LeComte-Hinely, my extremely intelligent and compassionate fellow cohort member, whose keen observations, good humor, and steadfast ambition have kept me motivated in this project as well as in my other work. I would like to acknowledge my family; in particular my parents and grandparents, whose supportive words throughout my graduate school career were much needed and always appreciated. Finally, I would like to acknowledge Alec Nielsen, whose endless encouragement and sunny disposition made the progression of this project all the more enjoyable. I am a better psychologist because of each of you. 
Table of Contents

Abstract pg.

Dedication pg. iii

Acknowledgements $\quad$ pg.iv

$\begin{array}{lll}\text { Chapter I. Introduction } & \text { pg. } 1\end{array}$

Chapter II. Supervisor-Subordinate Age Differences pg. 10

Supervisor-Subordinate Directional Age Differences pg. 15

Chapter III. The Quality of the Supervisor-Subordinate Relationship pg. 20

Chapter IV. Performance Feedback and Organizational Justice pg. 29

$\begin{array}{lll}\text { Chapter V. Employee Feedback Reactions } & \text { pg. } 38\end{array}$

Chapter VI. Hypothesis Development pg. 45

$\begin{array}{ll}\text { Study Hypotheses } & \text { pg. } 46\end{array}$

$\begin{array}{lll}\text { Chapter VII. Method pg. } 49 & \end{array}$

$\begin{array}{ll}\text { Participants and Procedure pg. } 49 & \text { pg }\end{array}$

$\begin{array}{ll}\text { Measures } & \text { pg. } 51\end{array}$

$\begin{array}{lr}\text { Chapter VIII. Results } & \text { pg. } 57\end{array}$

$\begin{array}{ll}\text { Factor Analyses } & \text { pg. } 57\end{array}$

$\begin{array}{ll}\text { Preliminary Analyses } & \text { pg. } 58\end{array}$

$\begin{array}{ll}\text { Structural Equation Models } & \text { pg. } 60\end{array}$

Exploratory Analyses of Feedback Valence Moderation pg. 72

$\begin{array}{lll}\text { Chapter IX. Discussion } & \text { pg. } 76\end{array}$

$\begin{array}{ll}\text { Hypothesized Relationships } & \text { pg. } 76\end{array}$ 
$\begin{array}{ll}\text { Exploratory Relationships } & \text { pg. } 92\end{array}$

Chapter X. Implications for Research and Practice pg. 101

Chapter XI. Limitations and Directions for Future Research pg. 114

$\begin{array}{ll}\text { Chapter XII. Conclusion } & \text { pg. } 124\end{array}$

$\begin{array}{lr}\text { References } & \text { pg. } 129\end{array}$

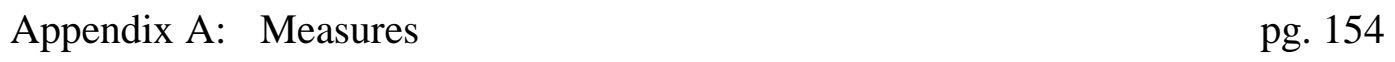

$\begin{array}{lll}\text { Table 1: } & \text { Inter-correlations among study variables } & \text { pg. } 158\end{array}$

$\begin{array}{lll}\text { Table 2: } & \text { SEM analysis path coefficients } & \text { pg. } 159\end{array}$

$\begin{array}{llr}\text { Figure 1: } & \text { Hypothesis } 1 \text { model } & \text { pg. } 162\end{array}$

$\begin{array}{lll}\text { Figure 2: } & \text { Hypothesis } 2 \text { model } & \text { pg. } 163\end{array}$

$\begin{array}{ll}\text { Figure 3: Hypothesis } 3 \text { model } & \text { pg. } 164\end{array}$

$\begin{array}{llr}\text { Figure 4: } & \text { Hypothesis } 4 \text { model } & \text { pg. } 165\end{array}$

Figure 5: $\quad$ Hypothesis 1 unstandardized (and standardized) analysis $\begin{array}{ll}\text { path coefficients } & \text { pg. } 166\end{array}$

Figure 6: Hypothesis 2 unstandardized (and standardized) analysis path $\begin{array}{ll}\text { coefficients pg. } 167 & \text { pg }\end{array}$

Figure 7: $\quad$ Hypothesis 3 unstandardized (and standardized) analysis path $\begin{array}{ll}\text { coefficients } & \text { pg. } 168\end{array}$

Figure 8: $\quad$ Hypothesis 4 standardized (and unstandardized) analysis path $\begin{array}{ll}\text { coefficients } & \text { pg. } 169\end{array}$

Figure 9: $\quad$ Full model standardized (and unstandardized) analysis path $\begin{array}{ll}\text { coefficients } & \text { pg. } 170\end{array}$ 
Figure 10: Feedback valence moderates the effect of supervisor-subordinate directional age differences on employee perceptions of LMX

pg. 171

Figure 11: Feedback valence moderates the effect of supervisor-subordinate directional age differences on employee feedback satisfaction

pg. 172

Figure 12: $\quad$ Feedback valence moderates the effect of supervisor-subordinate directional age differences on employee motivation following the feedback event pg. 173 


\section{CHAPTER I.}

\section{Introduction}

As the average workforce age increases and the relative number of younger workers decreases, the entry level labor pool is no longer made up exclusively of young (18 to 24 year old) adults (Greller \& Nee, 1989; Liebold \& Voelpel, 2006; Mor Barak, 2011). As a result, more organizations are hiring older workers into positions that were originally conceptualized as entry level. Consequently the pairing of an older employee with a relatively younger supervisor is becoming more and more common (Hirsch, 1990; Liebold \& Voelpel, 2006). Research has suggested that these dyadic patterns can lead to potential problems between supervisors and their subordinates due to the implied violation of age norms and the reversal of traditional age-related career development within organizations (Lawrence, 1984; 1988; Perry, Kulik, \& Zhou, 1999; Shore, Cleveland, \& Goldberg, 2003). As such, pairing a younger supervisor with an older subordinate may present difficulties in employee performance management.

Historically, one way that organizational entities have attempted to manage employee performance is through the provision of formal performance feedback. Performance feedback is typically delivered by immediate supervisors (Larson, 1989; Leung, Su, \& Morris, 2001) with the aim of building upon employees' strengths and reducing their weaknesses. The goal of formal performance 
feedback is to improve the overall performance of individuals, and thus of the entire organization (Kluger \& DeNisi, 1996). However, research and practice have shown that this does not always occur; $40 \%$ of performance feedback events are met with subsequent declines in performance rather than improvements (Kluger \& DeNisi, 1996).

As a result of such inconsistencies, feedback researchers have begun to focus on characteristics of feedback interactions and how these characteristics influence employee reactions and behavior over and above the impact of the performance rating itself. One such avenue of research has been focused on demographic attributes of supervisors and subordinates and how these impact attitudes and behaviors, both generally and in the context of performance feedback events. Empirical evidence indicates that supervisor-subordinate differences in gender, race, and age not only impact supervisor liking of a subordinate, generally leading to lower performance ratings, but also attitudes of the subordinate towards the supervisor and the feedback itself (Geddes \& Konrad, 2003; Liden, Stilwell, \& Ferris, 1996; Pelled \& Xin, 2000; Tsui \& O’Reilly, 1989; Varma \& Stroh, 2001; Vecchio, 1993).

Research on dyadic age differences in particular has indicated that while general age differences between oneself and one's supervisor tend to garner poorer employee attitudes (e.g., Riordan \& Shore, 1997; Tsui \& O’Reilly, 1989), 
age differences that also violate social norms are particularly influential in this respect. Put another way, having a younger supervisor-which violates "normal" career progression - has been shown to negatively impact: employee performance (Perry et al., 1999; Tsui, Porter, \& Egan, 2002); supervisor ratings and supervisor willingness to provide training and development opportunities (Shore et al., 2003); and employee work-related and supervisor-related attitudes (Collins, Hair, \& Rocco, 2009; Lawrence, 1984; Shore et al., 2003). Given the increasing incidence of non-normative dyadic relationships in the workforces of both developed and developing nations (Mor Barak, 2011), it is important for researchers to better understand these relationships in order to identify factorsespecially those that organizations can potentially influence - that minimize these negative effects.

Leader-member exchange (LMX), or the quality of the relationship between a supervisor and his or her subordinate, is a relational construct that research has consistently shown to be impacted by the demographic attributes listed above. Research has shown that supervisor-subordinate dyads that are similar in age, gender, and values tend to have higher-quality LMX (Epitropaki \& Martin, 1999; Graen \& Uhl-Bien, 1995; Pelled \& Xin, 2000). This idea is particularly important in the context of performance appraisals, given that LMX tends to result in higher ratings coming from the supervisor (Elicker, Levy, \& Hall, 2006; Wayne, Shore, 
Bommer, \& Tetrick, 2002) and better feedback reactions coming from the subordinates (Elicker et al., 2006; Levy \& Williams, 2004). Because LMX is important in determining employee attitudes regarding their supervisors and their work, organizational researchers and practitioners need to become more aware of how the changing nature of dyadic relationships impacts its development and progression.

Further, because LMX has been shown to buffer the negative individual and organizational effects of workplace politics (Harris \& Kacmar, 2005) and perceived organizational injustice (Tekleab, Takeuchi, \& Taylor, 2005), it is possible that the development of a high-quality relationship with one's supervisor can alleviate the negative impact of supervisor-subordinate age differences on employee attitudes and behaviors. As such, LMX may be one area for researchers to examine as they seek ways to minimize the harmful effects of demographically non-normative supervisor-subordinate pairings.

Another conceptual framework that has been used in the context of performance feedback characteristics research is organizational justice (Colquitt, 2001; Colquitt, Conlon, Wesson, Porter, \& Ng, 2001; Greenberg, 1990). Research has shown that justice during a feedback event, as experienced by the employee, will impact the employee's thoughts and feelings regarding the feedback event (e.g., Erdogan, 2002). In the case of supervisor-led feedback, employee 
interactional justice perceptions, or perceptions of how included and respected they feel within a performance appraisal event, become particularly important in impacting their subsequent attitudes regarding the feedback as well as their supervisor. Indeed, research has shown that perceived interactional justice in particular tends to predict a wealth of supervisor-directed attitudes and behaviors, as well as job performance (Cropanzano, Prehar, \& Chen, 2002; Erdogan, 2002). Further, research has shown these effects specifically in the context of performance appraisals (Erdogan, 2002; Leung et al., 2001).

Based on the preceding information, in the current study I examined how supervisor-subordinate directional age differences (i.e., whether the supervisor or subordinate was relatively younger) impacted employee reactions to a supervisorled performance appraisal event. Specifically, the aim of the study was to utilize an organizational justice framework in examining how relative age within a supervisor-subordinate dyad impacted employee feedback satisfaction, perceptions of feedback usefulness, and work motivation following the feedback event through the mediating mechanisms of employee interactional justice perceptions. Further, the role of LMX was examined as a mediator and moderator of these relationships, with the aim of enhancing researcher and practitioner understanding of how directional age differences impact LMX, and how various 
levels of LMX impact the relationships between these age differences and the various types of employee feedback perceptions and reactions.

The current study builds upon existing research in the topic area in several ways. First, this study was among the first of its kind to consider employee perceptions of and reactions to performance feedback in the context of unusual demographic dyad characteristics. While such "non-normative" situations have been tied to supervisor ratings of performance in the literature (e.g., Liden et al., 1996), virtually no research has examined the impact of having a younger supervisor on employee perceptions of the feedback. Given the growing importance of employee perceptions and reactions in understanding how and why performance feedback is at times ineffective, this conceptual link is important to examine.

Second, the current study simultaneously utilized an interactional justice approach and an LMX approach to study employee perceptions of and reactions to performance appraisal. Generally research has examined one or the other of these (e.g., Erdogan, 2002; Greenberg, 1986). However, given the increasingly recognized need for more broadly encompassing theories of leadership and employee performance (Barling, Christie, \& Hoption, 2011; Kelloway \& Barling, 2010; Leow \& Kuong, 2009), the current research aimed to examine these processes together, as they tend to naturally occur in the workplace. Further, in 
the current study I examined how supervisor-subordinate demographic patterns play a role in these processes, which is a necessary avenue of study given the rapidly changing composition of the global workforce.

The current study also employed a time-lagged component, which few employee feedback reactions studies have done in the past. I argue that performance feedback is administered with the aim of impacting employee attitudes and performance over time, rather than immediately. As such, the current research examined how dyadic age differences, employee justice perceptions, and LMX work together to influence employee reactions over time.

Finally, the current study explored these relationships in the context of a nation that is experiencing profound changes in the demographic patterns of its workforce, similar to those seen in the United States. Organizational researchers have frequently called for an increase of research in other nations and cultures besides the United States and other Western regions (e.g., Schaffer \& Riordan, 2003; Tsui, Nifadkar, \& Ou, 2007). Much of the existing feedback reactions literature in particular has been conducted in the United States or other Western cultures, with minimal inclusion of other nations. China, an Eastern culture, has been identified by economists as an economic super power, with strong ties between United States and Chinese businesses already occurring and projected to 
increase in the near future (Dessler \& Tan, 2006; Nolan, 2002; Yan \& Gray, 1994).

Despite the similarities in changing demographic trends, these two nations differ greatly in terms of cultural and organizational values, with China being more collectivistic, relationship-focused, accepting of power distance, and traditional in terms of respect for hierarchy and age (Child \& Markoczy, 1993; Dessler \& Tan, 2006; Farh, Tsui, Xin, \& Cheng, 1998; Fisher \& Yuan, 1998; Hofstede, 1980; 2001; Streib, 1987; Tsui \& O’Reilly, 1989). Increasing modernization and decreasing traditionalism in some regions have resulted in organizational functions and norms that are becoming more similar to those in the United States (Ayree \& Chen, 2006; Hui, Lee, \& Rousseau, 2004), but in many areas of China vast cultural differences still exist (Bailey, Chen, \& Dou, 1997; Dessler \& Tan, 2006; Hofstede, 2001; Tata, Fu, \& Woo, 2003).

Given the increasingly important role of globalization and cross-cultural collaboration in modern-day organizations, it is important that we better understand the processes and procedures inherent in Chinese business practices. As such, the current study adds to the current industrial/organizational literature in a conceptual sense, by examining the validity of relationships and theories previously established in a Western context in a different culture, and in a practical sense, by examining factors impacting employee attitudes and behaviors 
in a culture representing an important partner for United States economic functioning in a rapidly changing world of work.

In the following chapters I present the theoretical and empirical underpinnings of the current study, exploring how past research examining supervisorsubordinate age differences, LMX, interactional justice, and employee performance feedback reactions merges together to suggest the effects of supervisor-subordinate directional age differences on each of the study variables. Throughout these sections, I describe the values and norms inherent in Chinese business practice, and how these traditions impact the study variables and the hypothesized relationships. Study hypotheses are presented, as well as the current methods, the analyses and research findings, and a discussion of the results and their conceptual and practical implications for modern-day issues impacting the field of industrial/organizational psychology. 


\section{CHAPTER II.}

\section{Supervisor-Subordinate Age Differences}

Researchers have generally studied age differences within the supervisorsubordinate dyad using two frameworks. The first of these stems from the relational demography literature, while the second (discussed later) stems from implicit beliefs about demographic norms.

Relational demography is based on the attraction-similarity paradigm, which suggests that the more similar people are, the higher the degree of attraction between them (Riordan, 2000). In the context of the workplace, this theory suggests that people who are more similar to their coworkers in terms of demographic attributes such as age, gender, race, and job tenure should have better attitudes toward those coworkers. Additional research has indicated that such attitudinal outcomes can affect other things such as individual or team performance (e.g., Judge, Thoresen, Bono, \& Patton, 2001; Mowday, Porter, \& Dubin, 1974; Ostroff, 1992).

The idea of relational demography has received extensive theoretical and empirical support. Social Identity Theory (SIT; Tajfel \& Turner, 1986) suggests that people implicitly place themselves and others into social "groups" based on a variety of attributes including demographic elements such as gender, age, and race. Not surprisingly, researchers have found that people tend to exhibit an in- 
group favorability bias, by which they prefer members of their in-group to those of their out-group (e.g., Brewer, 1979; Brewer \& Kramer, 1985). As will be discussed below, this socio-cognitive theory has been applied in a number of research topic areas, including the study of workplace relationships and relational demography.

Tsui, Egan, and O’Reilly (1992), for instance, examined the impact of work group demography on employee attachment to the organization. As expected, increasing work group diversity in terms of age, tenure, education, sex, and race was associated with lower levels of attachment among group members. Similarly, Pelled (1996) found that among blue-collar workers, gender and tenure dissimilarity in work groups resulted in increased perceptions of emotional conflicts and decreased perceptions of group productivity, suggesting demographic dissimilarity might negatively impact employees' confidence in their work groups. Riordan and Shore (1997) examined work groups in a large life insurance organization and found that perceived dissimilarity from coworkers in terms of race and ethnicity was associated with poorer attitudes about those coworkers.

While most of the relational-demographic and SIT research has been done in Western societies and organizations, some has involved workers and organizations from non-western countries. For instance, Li and Hsu (1995) 
conducted a lab study in which they found that ideas about in-group and outgroup membership persist in Chinese samples as well as those in the United States. Moreover, Early (1993) found that Chinese participants who believed they were working on a task with members of their out-group as opposed to their ingroup performed more poorly, due to poorer perceptions of individual and group efficacy. One study containing a minority sub-sample comprised of Chinese and Japanese participants found that these participants experienced reduced workgroup fit and increased intent to turnover when they were demographically dissimilar to their work group (Kirchmeyer, 1995). These findings, as well as the research findings listed above, indicate that workers in various cultures prefer to be demographically similar to other members of their work group in order for them to feel positive about the group's functioning and confident in the group's abilities.

Similar evidence has been found for relational demography that is specific to the supervisor-subordinate dyad. For instance, Wessolowski and Mossholder (1997) found that being similar in race to one's supervisor tends to result in greater employee job satisfaction and greater perceptions of organizational justice. Tsui and O'Reilly (1989) found that dyadic differences in age, gender, race, education, and company and job tenure had multivariate negative effects on supervisor-administered employee performance ratings and supervisor liking of 
employees, while these differences had positive effects on employee role ambiguity. Likewise, Perry and colleagues (1999) found that greater demographic dissimilarity within the supervisor-subordinate dyad positively impacted employee absenteeism and negatively impacted employee citizenship behaviors. Exploring this effect in an Eastern culture, Farh and colleagues (1998) found that dyadic relational demography, as conceptualized by similarities in age, gender, and education, impacted employee trust in the supervisor in a Chinese sample.

Research has also shown that while actual demographic differences tend to impact important outcomes such as employee performance and supervisor ratings, perceived differences do as well (Turban \& Jones, 1988). As such, it appears that employees tend to have better attitudes towards their supervisor, and thus more positive organizational behaviors, when paired with a manager who is believed to be demographically similar.

Although relational demography has received support in the literature, other studies have revealed inconsistent effects of demographic dissimilarities in work groups and dyads. For instance, although Riordan and Shore (1997) found that work group differences in race and ethnicity impacted group-related attitudes, they found no effect for differences in gender or tenure. Further, some research evidence indicates that the issue of work-group demographic composition may be more complex than originally thought. Pearsall, Ellis, and Evans (2009) found 
that creative performance among gender-diverse teams of management students only suffered when gender-specific issues were explicitly made salient in the study task. Harrison, Price, and Bell (1998) suggested that demographic diversity negatively impacts work group integration, but only initially, and this effect disappears as the group has more time to engage in meaningful interactions. While Tsui and colleagues (1992) found that increased demographic diversity predicted lower levels of attachment among group members, this effect was much stronger for members of the majority (i.e., Caucasian males) and almost nonexistent for females and non-White group members.

Relational demography has also yielded inconsistent results in supervisorsubordinate dyads. For instance, Wessolowski and Mossholder (1997) found that racial differences in such dyads predicted reduced employee job satisfaction and perceived organizational justice, but no such effects were found for dyad age and gender differences. Similarly, and directly tied to performance appraisal, Geddes and Konrad (2003) examined dyadic demographic differences in a sample representing 120 nationalities and found that employees actually reacted more negatively to performance feedback that came from a supervisor of the same race. Further, while men reacted more negatively to feedback coming from female superiors, no such effect was found for female subordinates with male superiors, 
suggesting that social norms and roles may also play a part in employee perceptions of work relationships.

\section{Supervisor-Subordinate Directional Age Differences}

From the above studies, it becomes clear that while demographic characteristics help to explain some variance in employee attitudes and behaviors, the relationship is more complex than simple similarities and differences. One line of research stemming from these inconsistencies that is especially influential in understanding the effects of supervisor-subordinate age differences (and as such is the second major framework researchers use to study this phenomenon) adds perceptions of normative career progression.

Lawrence (1984) suggested that people form implicit "timetables" of normal career progression and tend to judge careers as being on or off schedule according to these perceptions. While these perceptions are not always accurate, they have been shown to influence employee work attitudes. For instance, perceptions of the adequacy of one's career stage have been linked to career and work motivation (Noe, Noe, \& Bachhuber, 1990) and job satisfaction (Lawrence, 1984). Lawrence (1984) found that managers who perceived themselves as being "behind" in terms of their career progression experienced more negative work attitudes and were less oriented toward work, regardless of the accuracy of those perceptions. Not surprisingly, the most common marker by which people determine normal career 
progression is chronological age (Lawrence, 1984; Sofer, 1970). As such, researchers have begun focusing more directly on directional age differences within supervisor-subordinate dyads. Where the study of general age differences calls for an examination of the degree of difference or similarity in supervisor and subordinate ages (operationalized, for instance, as the absolute value of the difference between the two ages, e.g., Perry et al., 1999; Turban \& Jones, 1988), studies examining directional age differences have operationalized these by explicitly asking employees to indicate whether they perceive their supervisor as being younger or older or by subtracting supervisor chronological age from employee age and exploring patterns occurring on the positive and negative side of the difference (e.g., Perry et al., 1999; Shore et al., 2003; Vecchio, 1993). The idea in this line of research is that if people use age as a marker for determining their place on their implicit career timetable, having a younger supervisor in particular should represent a violation of this and thus should result in poorer employee outcomes.

Empirical evidence has largely supported this notion. Shore et al. (2003) found that employees who were older than their managers received more negative performance evaluations and fewer opportunities for training and development. Employees with younger managers were also found to have less favorable work attitudes (Shore et al., 2003), despite the generally positive relationship between 
employee age and job attitudes (Ng \& Feldman, 2010). Perry and colleagues (1999) found that while age similarity within the dyad accounted for some variance in employee absenteeism and citizenship behaviors, directional age differences in the dyad (i.e., having a younger supervisor in particular, noted here as "status incongruence") accounted for far more of the variance in these behaviors. Collins and colleagues (2009) found that older workers tend to have lower expectations of their younger supervisors, and in turn tend to rate younger supervisors' leadership behaviors lower. Although one study suggested that older employees reported better working relationships with younger supervisors and evaluated those supervisors more favorably (Vecchio, 1993), this study was conducted using a sample entirely comprised of high school faculty members, an occupational group in which career progression is not necessarily demarked by age (i.e., having the training and education necessary to become a teacher is not the same as what is necessary to become a principal or superintendent, so career progression does not occur linearly here). By and large, the research supports the idea that employees possess implicit age-driven expectations about career progression (Lawrence, 1984), and having a younger supervisor generally violates these expectations.

Implicit Leadership Theories (ILT) is one string of theories that may help to explain this phenomenon. According to ILT, people possess implicit ideas of what 
a leader should be, and these cognitions are likely to shape their attitudes and perceptions regarding leaders they come across (Lord, DeVader, \& Alliger, 1986; Lord, Foti, \& DeVader, 1984; Offermann, Kennedy, \& Wirtz, 1994). While this encompasses characteristics such as leader behavior and personality (Lord et al., 1986; Offermann et al., 1994), it also encompasses demographic features (Epitropaki \& Martin, 2005). Implicit career timetables research indicates that chronological age is one such feature (Lawrence, 1984), such that a "normal" or "ideal" supervisor is relatively older.

ILT have received support in research specific to Chinese organizations. Such research has shown that the type of leader that garners the most positive employee reactions in China is typically interpersonally competent, authoritarian, moral, versatile, and wise (Cheng, Chau, \& Wu, 2004; Ling, Chia, \& Fang, 2000), which are characteristics expected of older Chinese citizens (Hofstede, 1980; 2001). Further, given the emphasis placed on power distance in China and many Chinese organizations (Brockner, Ackerman, Greenberg, \& Gelfand, 2001; Hofstede, 2001; Tata et al., 2003), it stands to reason that the ideal Chinese leader is relatively older, wiser, and more experienced.

Despite these perceived norms, the incidence of demographic norm "violations" is increasing globally, in American and Chinese organizations alike (Hirsch, 1990; Lawrence, 1998; Liebold \& Voelpel, 2006). However, relatively 
little research has examined the effects of these violations on employee attitudes

in China. Given the increasing importance of Chinese businesses in the globalized economy, there is a need for organizational researchers to address the impacts of these perceived violations in China, and identify ways in which their negative effects may be mitigated. 


\section{CHAPTER III.}

\section{The Quality of the Supervisor-Subordinate Relationship}

Perhaps the most comprehensively studied construct in the context of the supervisor-subordinate dyad is leader-member exchange (LMX). LMX is a leadership theory that takes into account not just the leader's behaviors and performance, but also attitudes of the follower (Graen \& Uhl-Bien, 1995). As such, LMX is a relationship-based approach to leadership and refers broadly to the quality of the relationship in the supervisor-subordinate dyad (Bauer \& Green, 1996; Graen \& Uhl-Bien, 1995; Schreisheim, Castro, \& Cogliser, 1999). Stemming originally from the theory of Vertical Linkage Dyad (VLD; Dansereau, Graen, \& Haga, 1975; Graen \& Cashman, 1975), LMX theory takes into account multiple dimensions where supervisor-subordinate relationship functioning takes place.

While historically LMX was assessed in research with one or two items asking subordinates to indicate how they felt about the relationship between themselves and their supervisors, more recent LMX measurement accounts for those multiple dimensions (Schreisheim et al., 1999). For instance, the LMXMDM, or multi-dimensional, survey contains multiple items that encompass subordinate and supervisor contribution (the perception of the amount, direction, and quality of work-oriented activity each member of the dyad puts forth toward 
mutual goals), and loyalty, or the extent to which both members of the dyad support each other publicly. The scale also includes affect items, assessing the mutual attraction each member of the dyad perceives based on interpersonal attraction rather than work or professional values (Liden \& Maslyn, 1998). Another example is the LMX-7 form developed by Scandura and Graen (1984). This survey requires participants to indicate their perceptions of the supervisorsubordinate relationship using seven items that encompass the working relationship, supervisor understanding and support of the subordinate outside the working relationship, and supervisor recognition of the subordinate. Further subdimensions that have been suggested as being part of the LMX construct are opportunities for subordinate influence and control, trust, quality of interpersonal exchange, and assistance and support (Scandura, Graen, \& Novak, 1986; Schreisheim et al., 1999).

LMX theory suggests that when employees perceive the dimensions above as occurring in their relationships with their supervisors, they perceive high-quality LMX. While LMX has generally been assessed by obtaining scores of employee perceptions of the quality of the dyadic relationship, more recent research has also moved toward assessing the perceptions of both members of the dyad in order to get a more complete picture of LMX (e.g., Schreisheim et al., 1999). 
In their comprehensive review of LMX research, Graen and Uhl-Bien (1995) suggested that this topic area has progressed through four major stages. The first stage, largely exploratory, examined the then-revolutionary idea that while overall leadership style is important, it is also important to consider that leaders employ a slightly different style with each subordinate. Put another way, supervisors tend to have different relationships with each of their employees instead of utilizing one main style with all of them, which at the time this idea was put forth had not yet been considered (Dansereau et al., 1975).

The second major phase involved the building up of the nomological net surrounding LMX. In this phase the antecedents, outcomes, and correlates of LMX were established, and this is where most of the major research has taken place. Such research has revealed that relationship tenure (the longer the dyad has been working together) positively predicts LMX (Schyns, Paul, Mohr, \& Blank, 2005), along with trust that goes beyond the formal employment contract (Dienesch \& Liden, 1986). Similarly, it has been suggested that demographic similarities in terms of gender and personality can also contribute directly to the formation of LMX, as well as indirectly through the formation of trust and the delegation of important tasks from the supervisor to the subordinate (Bauer \& Green, 1996; Dienesch \& Liden, 1986). 
Building on this, relational demography research shows that a greater degree of similarity in general tends to predict more positive employee attitudes (e.g., Perry et al., 1999; Tsui et al., 1992; Tsui \& O’Reilly, 1989), and LMX may well be one of these attitudes (Pelled \& Xin, 2000). Moreover, relational demography research suggests that these long-lasting relationships are likely to stem from initial similarity and attraction since we tend to be attracted to and stay with people who are similar to us, provided that voluntary turnover is an option (Milliken \& Martins, 1996; Schyns et al., 2005). Social Identity Theory (SIT; Tajfel \& Turner, 1986) may again provide some insight into why this may be the case; people who are more similar to us tend to be perceived as members of our in-group and thus tend to be better liked (Brewer, 1979; Brewer \& Kramer, 1985; Kirchmeyer, 1995). Dissimilar people, on the other hand, yield less interpersonal attraction, so a high-quality relationship will likely be more difficult to develop. Indeed, supervisors that are more demographically similar to their employees tend to get higher LMX ratings from those employees (Epitropaki \& Martin, 1999; Pelled \& Xin, 2000). While relational demography and LMX have not been explicitly studied in China, research has shown that demographic similarity is important in predicting employee trust in the supervisor (Farh et al., 1998). Further, in highly collectivistic organizations like many of those found in China, 
demographic similarity to the supervisor has some bearing on predicting employee promotions (Schaubroeck \& Lam, 2002).

This framework can also be applied to understanding attitudes and relationships that form among supervisor-subordinate dyads that are demographically unusual. For one thing, there is some research to indicate that the idea of implicit career timetables relates to LMX. Specifically, as previously mentioned, research shows that directional age differences in a dyad (i.e., having a supervisor that is younger than oneself) yield poorer employee reactions regarding that individual and regarding their work in general (Lawrence, 1984; Perry et al., 1999). Also previously mentioned, ILT suggest that a younger supervisor could be considered as being outside of an employee's perceived social norms for self- and other-identity (Lawrence, 1984; Lord et al., 1986; Offermann et al., 1994).

Research examining directional demographic differences and their impact on LMX among Chinese employees specifically has not been undertaken to date. This research gap unfortunately leaves organizational psychologists uninformed as they seek to understand supervisor-subordinate relationships in Eastern cultures. However, there is some reason to believe that directional age differences impact the formation of LMX in Chinese dyads. Age-related cultural norms emphasizing age as a marker of expertise, experience, and wisdom suggest that 
Chinese employees will be more satisfied with an older superior (Farh et al.,1998; Hofstede, 1980; 2001). As such, the current research aimed to delve more concretely into the impact of directional age differences on the formation of a positive supervisor-subordinate relationship.

LMX is important to include in such research from an organizational standpoint, as it has been found to correlate significantly with a number of important outcomes such as increased supervisor trust in the subordinate's abilities, subordinate attitudes toward the supervisor and work in general, subordinate affective commitment, and subordinate self-efficacy at work (Schyns et al., 2005). Further, LMX has been shown to negatively predict subordinate turnover (Graen, Liden, \& Hoel, 1982), and to positively predict subordinate satisfaction (Graen, Novak, \& Sommerkamp, 1982), subordinate promotions (Wakabayashi, Graen, Graen, \& Graen, 1988), and subordinate extra-role performance (Wayne \& Green, 1993).

While very little research has specifically examined dyad demographics and LMX simultaneously in China, much Chinese organizational research has demonstrated the importance of LMX in the workplace in general. Research has suggested that employee perceptions of the supervisor having cooperative rather than competitive goals predict LMX, which in turn predicts organizational citizenship behaviors such as altruism and courtesy (Hui, Law, Chen, \& Tjosvold, 
2008). Other antecedents to LMX in China include the leader's power and a supportive work climate, with the resulting LMX being shown to predict intrinsic task motivation, employee job satisfaction, and employee task performance (Ayree \& Chen, 2006). Another LMX study conducted in China found that multidimensional LMX comprised of affect, loyalty, contribution, and professional respect was a strong predictor of both task and contextual employee performance (Hui, Xiongying, \& Law, 2004). China-focused research has even found that LMX, as well as team-member interaction, is an important predictor of effective new-hire onboarding and socialization initiatives (Lam, 2003).

Perhaps greatest practical contribution of LMX in the workplace is that its presence can help to buffer otherwise potentially negative work characteristics. Research has shown that the negative impact of unfavorable work environments can be tempered by the resource of having a good relationship with one's supervisor (e.g., Bakker, Demerouti, \& Euwama, 2005). For instance, Harris and Kacmar (2005) found that the negative straining impact of workplace politics in an organization was buffered by LMX and better communication with supervisors. Further, Masterson, Lewis, Goldman, and Taylor (2000) found that while procedural justice perceptions impact employee perceptions of the organization, employee perceptions of interactional justice tend to impact their attitudes about their supervisors, and thus lead to positive organizational 
outcomes such as task and extra-role performance even in the face of poor procedural justice coming from the organization (for a more complete description of organizational justice, please see the section below).

Numerous empirical studies have demonstrated the positive outcomes stemming from LMX (e.g., Graen et al., 1982; Schyns et al., 2005; Wayne \& Green, 1993), and outcomes such as employee job satisfaction, performance, and extra-role behavior have been found specifically in Chinese organizational research (e.g., Ayree \& Chen, 2006; Hui et al., 2004; 2008). As such, it is possible that if a high-quality LMX relationship can form in a dyad in which implicit career timetables are violated, this can help to alleviate the negative impact that research suggests such a relationship may have on employee attitudes and behaviors (Lawrence, 1984). Pertinent to the current study, one particular context in which these negative attitudes may affect the quality and effectiveness of a supervisor-subordinate interaction is in the case of supervisor-led formal performance appraisal (Duarte, Goodson, \& Klich, 1994).

The above correlates formed and continue to form the nomological net around LMX. Building on these, the third major phase of LMX examination (Graen \& Uhl-Bien, 1995) involved studying ways in which leaders can become better able to promote high-quality LMX relationships. Research in this vein has shown that organizations and leaders can seek to develop an internal locus of control among 
employees (Martin, Thomas, Charles, Epitropaki, \& McNamara, 2005), promote high-quality relationships among supervisors and upper management (Venkataramani, Green, \& Schleicher, 2010), and promote perceived organizational support (Wayne, Shore, \& Liden, 1997) when seeking to build LMX within their supervisor-subordinate dyads.

The fourth and most recent research phase of LMX involves studying these dyadic relationships in the context of the larger organizational and societal system (Graen \& Uhl-Bien, 1995), which LMX research in China has begun to do by incorporating cultural and societal values and norms (Hui et al., 2004; 2008). The current study incorporated elements of both cultural and organizational contexts in forming and affecting LMX, as dyadic relationship quality was examined in a Chinese sample whose members had recently been administered annual performance appraisals. 


\section{Chapter IV.}

\section{Performance Feedback and Organizational Justice}

Performance feedback is a widely-used method of managing employee performance in organizations (Cascio \& Aguinis, 2005). Informally, new and tenured employees may seek information regarding the quality of their work performance, or supervisors may offer colloquial feedback to subordinates in the context of certain projects or tasks (Farr, 1993). Formally, performance feedback is administered in the form of an appraisal or rating and occurs at regular time intervals (Cascio \& Aguinis, 2005). Although many organizations are moving toward a 360-degree system in which employees receive feedback from their supervisors, coworkers, customers, and selves (e.g., Brett \& Atwater, 2001), generally it is still the supervisor who is charged with the task of administering the ultimate appraisal to an employee (Larson, 1989; Leung et al., 2001). Research regarding interactions between supervisors and subordinates during performance feedback is, therefore, helpful in determining how to craft effective feedback.

The need to improve our understanding of effective and ineffective feedback has been brought to light by a number of researchers (Balcazar, Hopkins, \& Suarez, 1986; Brett \& Atwater, 2001; Ilgen \& Davis, 2000; Kluger \& DeNisi, 1996) and efforts to do so have spanned a large number of studies and research 
areas. Historically, feedback was conceptualized as a means of rewards and punishments. Specifically, it was thought that positive feedback would act as a reward and thus would result in an increase of desired work behaviors.

Conversely, as per Thorndike's Law of Effect (1929), negative feedback would act as a punishment and thus would result in the cessation of undesired work behaviors. Researchers have found this notion to be largely inconsistent with empirical and real-world findings. As previously mentioned, employees often respond to feedback with a lack of subsequent performance change (Brett \& Atwater, 2001), or even worse, employee performance sometimes declines following feedback (Kluger \& DeNisi, 1996). As a result of such findings, researchers began to look to other elements of feedback events besides simply their negativity or positivity. One element that has become a performanceappraisal research focus is organizational justice.

Organizational justice, in general, refers to employee perceptions of how they are treated by the organization they work for (Colquitt et al., 2001). Justice is a multi-dimensional construct that encompasses both perceptions of fairness of outcomes compared to inputs (distributive justice) and perceptions of fairness of the processes and procedures used to select those outcomes (procedural justice). More recently, the dimension of procedural justice has been subdivided to include interactional justice, which refers more concretely to interpersonal fairness that 
employees experience at work. Specifically, it is suggested that employees receiving high-quality and accurate information regarding outcomes and procedures at work (informational justice, a sub-component of interactional justice) and fair and respectful treatment from organizational entities such as supervisors (interpersonal justice, another sub-component) will perceive higher interactional justice and thus have better individual and work outcomes. Indeed, research has shown an influence of each of the justice dimensions, with higher levels of perceived justice on one or more dimensions being linked to job satisfaction, organizational commitment, performance, health, and citizenship behaviors (Colquitt, 2001; Colquitt et al., 2001; Greenberg, 1990).

Organizational justice has also been shown to influence employee attitudes specific to performance appraisals. For instance, Greenberg (1986) found that both procedural and distributive elements predicted how fair people found performance feedback events to be. Specifically, employees tended to rate feedback as being fairer when it involved some sort of two-way communication during the review, when they had the ability to challenge the appraisal, and when there was a consistent application of standards. Similarly, Erdogan (2002) found that the impression-management behaviors of appraisers (an interactional construct) predicted how fair respondents reported performance appraisals to be. 
Interactional justice has been proposed as an especially important justice dimension in perceptions of the supervisor-subordinate relationship, as this type of justice influences perceived supervisor honesty, trustworthiness, and respect (e.g., Cropanzano et al., 2002). Distributive and procedural justice, on the other hand, tend to be more related to perceptions of organizations as entities (Colquitt et al., 2001). There is some evidence that perceived interactional justice tends to predict employee attitudes toward dyad performance-related interactions specifically, possibly through the mechanism of social exchange norms (Cropanzano et al., 2002). Specifically, social exchange theory suggests that people often act based on their perceptions of reciprocal obligations created by the behavioral and attitudinal inputs that others direct toward them (Cropanzano et al., 2002; Emerson, 1976). In the context of a supervisor-subordinate dyad, employees perceive the degree to which their supervisor likes or cares for them and, as a result, adjust their behaviors toward that supervisor accordingly (Wayne et al., 1997). If employees perceive that their supervisor has shown them a high degree of interactional justice within a supervisor-led performance appraisal event, this should lead to positive, reciprocal exchange, including increased motivation to perform well.

While the bulk of organizational justice research has been done in the United States and other Western cultures, there is some evidence that it may be an 
important construct in the feedback reactions of Chinese employees as well (e.g., Dessler \& Tan, 2006). The limited available research has shown that both distributive justice elements, such as fair pay, and procedural justice elements, such as fair processes used to determine promotions, predict job satisfaction among Chinese employees (Leung, Smith, Wang, \& Sun, 1996). Interactional justice elements have also been specifically studied relative to performance appraisal reactions among Chinese workers. Such studies have found that, apparently because of the Chinese cultural value emphasizing relationship building and mutual respect (Child \& Markoczky, 1993; Dessler \& Tan, 2006; Fisher \& Yuan, 1998; Hofstede, 1980; 2001), Chinese employees respond more positively to performance feedback when the supervisor is open, honest, and direct (Chow, 1995). Further, research has indicated that the principle of "social sensitivity" may be particularly important in forming Chinese employee reactions to performance appraisal events (Tata et al., 2003).

As with organizational justice, researchers have suggested that leader-member exchange (LMX) operates through social exchange, such that employees perceiving a better relationship with their supervisor will "repay" them with more positive attitudes and behaviors. Research has shown, for instance, that employees who perceive themselves as having relatively good LMX also tend to exhibit better performance, less turnover, and fewer withdrawal behaviors, along with 
experiencing increased job satisfaction and well-being (Epitropaki \& Martin, 2005; Ferris, 1985; Graen et al., 1982; Wayne et al., 2002).

Overall, interactional justice and LMX are highly correlated; researchers have produced evidence that some components of interactional justice, such as leader honesty and consistency, are vitally important in the formation and maintenance of LMX (Scandura, 1999). Research has also indicated that higher-quality LMX within a dyad is associated with more positive employee attitudes regarding performance ratings (Levy \& Williams, 2004). Thus, it stands to reason that employees perceiving high-quality LMX with their supervisors should also have positive perceptions of the interactional justice shown to them by that supervisor within a performance feedback event. Indeed, research has supported this; employees who perceive better LMX tend to rate their supervisors as exhibiting more fairness in performance feedback (Erdogan, 2002; Scandura, 1999). While the relationship between LMX and employee feedback justice perceptions has not been explicitly studied in China, Chinese cultural emphases on relationship building and mutual respect indicate that more just, respectful supervisory behaviors should correlate positively with LMX in a similar manner.

Pertinent to the current study, some research evidence has also indicated that demographics within the supervisor-subordinate dyad can impact supervisor justice behaviors and employee justice perceptions. Research has shown that after 
controlling for objectively-assessed performance, both male and female supervisors tend to exhibit a positive bias toward subordinates of the same gender (Varma \& Stroh, 2001), supporting the notion that in-group/out-group demographic patterns play a significant role in outcomes of the supervisorsubordinate dyad. A study conducted by Geddes and Konrad (2003) found that all employees, on the other hand, preferred performance feedback coming from a normatively majority-status supervisor (e.g., an older, white male in the United States).

Again, the types of findings outlined above not been studied among Chinese workers. However, research in China has indicated that greater demographic similarity in the supervisor-subordinate dyad promotes employee trust in the supervisor (Farh et al., 1998). That supports the possibility of dyadic demographic patterns influencing LMX and employee justice perceptions in China.

Together, these studies and the ones presented above indicate that dyadic demographic make-up and supervisor-subordinate relationship quality do impact employee attitudes regarding feedback. However, while there has been speculation about the role of relationships and relational demography in employee attitudes in general (e.g., Riordan, 2000), an empirical link has not been 
documented among directional demographic similarities or differences, LMX patterns, and employee feedback reactions, in the United States or otherwise.

As with LMX, some recent research indicates that justice perceptions can be a mechanism through which dyadic demography impacts employee feedback reactions. For instance, research has suggested that relational demography impacts employee justice perceptions, with racial dissimilarity within a supervisor-subordinate dyad resulting in reduced employee perceptions of procedural justice at work (Wesolowski \& Mossholder, 1997). Further, Naumann and Bennett (2000) found that demographic similarity within work groups positively predicted perceptions of procedural justice climate within those groups. Also, and as previously noted, violations of normative career timetables resulting from having a younger supervisor tend to yield poorer supervisor-focused attitudes (Collins et al., 2009; Lawrence, 1984; Perry et al., 1999), which may likely include employee impressions of how fair their supervisors are during dyadic interactions. In Chinese organizations, this effect might be especially strong (though this has not been directly researched to date) due to Chinese cultural values espousing chronological age as a marker of wisdom and expertise (Child \& Markoczy, 1993; Dessler \& Tan, 2006; Hofstede, 1980; 2001; Ling et al., 2000). In the next section I discuss in greater detail employee reactions to 
performance feedback, and how justice perceptions play a part in how such reactions are formed. 


\section{CHAPTER V.}

\section{Employee Feedback Reactions}

Researchers examining employee reactions to performance feedback propose that certain elements of feedback events - particularly valence, or the degree to which the feedback is overall positive or negative, and perceptions of justiceimpact employee attitudes and cognitions, which then determine how effective or ineffective feedback will be in improving performance (Baron, 1993; Brett \& Atwater, 2001; Ilgen, Fisher, \& Taylor, 1979; Kluger \& DeNisi, 1996; Pearce \& Porter, 1986).

The feedback-reactions literature has generally focused upon three types of employee reactions: affective, cognitive, and motivational. Affective reactions refer broadly to how a feedback event makes an employee feel. Such reactions have been measured by assessing the degree to which employees were retroactively satisfied with the feedback they received (e.g., Dobbins, Cardy, \& Platz-Vieno, 1990). Cognitive reactions refer broadly to what an employee thinks about the feedback he or she has received. Such reactions have been measured by assessing employee perceptions of how useful the information contained in the feedback event was, as well as their perceived ability to transfer what they learned in the feedback back to their jobs (Baron, 1993; Brett \& Atwater, 2001; Podsakoff \& Farh, 1989). Cognitive feedback reactions have also been measured by 
assessing the degree to which employees perceived the feedback as being accurate in describing their work performance (e.g., Brett \& Atwater, 2001). Finally, motivational reactions refer to how motivated (or energized) employees feel in their jobs as a result of the performance feedback event (i.e., how their motivation level is affected by the feedback). Not surprisingly, these three types of employee reactions are generally highly correlated, with people perceiving more accurate, useful feedback as being more satisfying and motivating (Brett \& Atwater, 2001; Burlacu, Wang, James, Truxillo, \& Yao, 2012). Research suggests that feedback givers should attempt to achieve positive levels of each of these types of employee reactions, as greater employee feedback satisfaction, perceptions of usefulness, and work motivation are proposed to enhance performance appraisal effectiveness in the form of improved employee performance (e.g., Bianchi \& Ames, 2008; Brett \& Atwater, 2001; Pearce \& Porter, 1986).

Performance feedback valence (whether the feedback is overall positive or negative) tends to have a large main effect on all three employee reactions. This supports self-enhancement theory (Shrauger, 1975), and empirical research derived from it, that indicates that people's evaluations of their own performance tend to be relatively high. As such, employees receiving correspondingly high ratings that match with these positive self-evaluations are fulfilling their need for self-enhancement (Mabe \& West, 1982). In line with that claim, more positive 
feedback predicts feelings of pleasantness and pride, as well as satisfaction with the appraisal and the appraisal process (Brett \& Atwater, 2001; Kluger \& DeNisi, 1996; Mabe \& West, 1982; Shrauger, 1975). Positive feedback is also generally perceived as being more useful (Baron, 1993; Podsakoff \& Farh, 1989) and credible (Brett \& Atwater, 2001). Negative feedback, in contrast, has been associated with negative arousal, cognitive dissonance, and mistrust (Brett \& Atwater, 2001; Festinger, 1954; Kluger \& DeNisi, 1996; Leung et al., 2001; Taylor, Fisher, \& Ilgen, 1984), and has been shown to be potentially motivating only when the feedback is perceived as being credible (Podsakoff \& Farh, 1989). Taken together, these studies indicate that people generally have better reactions when receiving positive feedback regarding their work performance.

Above and beyond the effects of valence, there is some evidence that employee perceptions of justice as experienced during the feedback event also influence their satisfaction, perceptions of feedback usefulness, and post-feedback work motivation. Employees treated justly perform better and have more positive job satisfaction, organizational commitment, and attitudes toward their supervisors in general (Colquitt, 2001; Colquitt et al., 2001; Masterson et al., 2000). Specific to performance feedback, an appraisal system perceived to be more fair has been shown to predict more favorable reactions toward the feedback 
process and toward managers, as well as higher intentions to remain with the organization (Taylor, Tracy, Renard, Harrison, \& Carroll, 1995).

Fairness Heuristic Theory (FHT; Van den Bos, Lind, \& Wilke, 2001) may provide some insight into how justice perceptions impact performance feedback reactions. FHT suggests that information that is fairer is not only more likely to be noticed and to be given more weight in judgment and decision-making processes, but also more likely to positively impact attitudes and motivation (Van den Bos, Wilke, \& Lind, 1998; Van den Bos et al., 2001). As such, employees perceiving fairness during a performance feedback event are likely to have better affective, cognitive, and motivational reactions to the feedback.

Again, research has largely supported this idea, particularly in the domain of interactional (informational and interpersonal) justice. Recent researchers have examined feedback content and delivery — two constructs very similar to informational and interpersonal justice — and their impact on employee feedback reactions. Content, which refers to the quality of relevant information presented during a feedback event, was been found to predict employee satisfaction, perceptions of feedback usefulness and credibility, and performance motivation (Bianchi \& Ames, 2008). Similarly, delivery—which refers to the extent to which the feedback giver is polite, honest, encouraging, and prepared—was also found to predict all of those types of reactions (Bianchi \& Ames, 2008; Burlacu et al., 
2012). Research has also shown directly that higher perceived informational justice predicts employee motivation (Roberson \& Stewart, 2006), while higher perceived interpersonal justice yields more positive reactions to feedback and managers even when feedback is negative (Leung et al., 2001). Thus, employee justice perceptions, as well as valence, appear to be important mechanisms guiding their reactions to performance feedback.

Research conducted specifically in Chinese organizations also indicates that justice perceptions impact work-related attitudes. For instance, in Fisher and Yuan (1998), distributive justice elements such as good wages and good working conditions were found to predict work motivation among Chinese employees. Fisher and Yuan (1998) found similar results from procedural justice elements such as loyalty from the boss and the organization, and interactional justice elements such as perceptions of a good general supervisor-subordinate relationship. In another study, distributive and procedural justice elements such as perceptions of fair pay and fair promotional procedures were found to predict employee job satisfaction in a hotel chain in China (Leung et al., 1996).

Some research has also shown the importance of justice specifically in the context of performance feedback reactions. For instance, Chow (1995) found that open, direct communication from one's supervisor during feedback administration was preferred in organizations in the People's Republic of China, but that 
interpersonal and informational justice had differing effects on employees' overall reactions to performance feedback. While both of these justice elements had some bearing on how employees perceived a feedback event (Chow, 1995), interpersonal justice appeared to better match Chinese norms of having a good relationship with one's supervisor and being treated with respect (Brockner et al., 2001; Tata et al., 2003). Regardless, particularly in Chinese regions that are becoming increasingly less traditional and more modernized (e.g., Ayree \& Chen, 2006), both interactional justice elements have been found to carry weight in predicting employee feedback reactions and work-related attitudes in general (e.g., Brockner et al., 2001; Chow, 1995; Fisher \& Yuan, 1998).

The role of feedback valence in predicting perceptions of and reactions to performance appraisal among Chinese employees is less clear. On the one hand, important values in China include "preservation of face" and performing well for the organization (Child \& Markoczy, 1993), indicating that performance ratings, or feedback valence, should be important to Chinese employees. On the other hand, performance feedback coming from one person (the supervisor) is generally individually-focused (Hempel, 2008). Chinese values emphasizing a collectivistic, group orientation may result in these individualized performance ratings being less important, as they do not necessarily reflect an employee's contribution to the larger group (Child \& Markoczy, 1993). 
Further, the degree to which these ratings are taken seriously may differ depending on employee perceptions of the quality of the supervisor-subordinate relationship. One study found that when Chinese employees perceived poor LMX with their supervisors, negative feedback was perceived as a message about the relationship rather than a message about their own poor performance (Hempel, 2008). Thus, in this case, negative feedback may not have been taken seriously by employees as a reflection of their true job performance. These findings suggest that if, as argued earlier, Chinese employees have more negative attitudes toward their relatively younger supervisors, feedback valence may have little bearing on their affective, cognitive, or motivational reactions to performance feedback. 


\section{CHAPTER VI.}

\section{Hypothesis Development}

The preceding theoretical and empirical arguments suggest that having a relatively younger supervisor-representing a demographically "non-normal" pairing according to the tenets of implicit career timetables and Implicit Leadership Theories (e.g., Lawrence, 1984; Lord et al., 1984; 1986)—should have a negative impact on employee attitudes, particularly in the case of supervisordriven feedback events where supervisor-related attitudes are present and salient (e.g., Levy \& Williams, 2004). The current study aimed to examine this, and to explore a variety of potential mediating and moderating mechanisms that may contribute to that particular relational-demographic effect. Further, the current study attempted to disentangle these relationships in the context of a Chinese organization. China is a nation experiencing rapid demographic shifts (e.g., an aging population) that are similar to those occurring in the United States, but whose cultural values and norms differ from those in the U. S. As indicated in my literature review, Chinese values may cause a non-normative youngersupervisor/older-subordinate pairing to produce even more extreme effects on performance appraisal reactions than would occur elsewhere.

While the potential relational-demographic relationship just outlined was an explicit hypothesis of the study (see hypotheses below), the current study also 
targeted a more open-ended research question. That is, given the unclear role of feedback valence in predicting employee attitudes and behaviors following a feedback event in Chinese organizations, the study included an exploratory examination of how valence might also impact reactions to performance feedback in China. While the ILT and implicit career timetables literatures (Lawrence, 1984; Lord et al., 1984; 1986) suggest that the negative impact of non-normative demographic pairings should be pervasive, regardless of whether feedback is positive or negative, research findings and theoretical tenets from the "West" point to the possibility of valence as moderating feedback reactions (e.g., Brett \& Atwater, 2001; Kluger \& DeNisi, 1996; Mabe \& West, 1982; Shrauger, 1975). As such, exploratory analyses in the current study attempted to shed some light on the previously unstudied effects of feedback valence on the performance feedback event reactions of Chinese employees paired with supervisors corresponding to (relatively older) and violating (relatively younger) cultural norms.

\section{Study Hypotheses}

Hypothesis 1. Supervisor-subordinate directional age differences will negatively predict employee feedback reactions, such that having a younger supervisor will significantly and negatively predict (a) employee feedback satisfaction, (b) employee perceptions of feedback utility, and (c) employee 
motivation following the feedback event. Please see Figure 1 for a visual representation of this hypothesis.

Hypothesis 2. Employee interactional justice perceptions will mediate the relationship between supervisor-subordinate directional age differences and employee feedback reactions, such that (a) having a younger supervisor will predict lower ratings of informational justice, and informational justice scores will at least partially mediate the directional age differences effects to (b) employee feedback satisfaction, (c) employee perceptions of feedback utility, and (d) employee motivation following the feedback event. Moreover, (e) having a younger supervisor will predict lower ratings of interpersonal justice, which will at least partially mediate effects on (f) employee feedback satisfaction, (g) employee perceptions of feedback utility, and (h) employee motivation following the feedback event. Please see Figure 2 for a visual representation of this hypothesis.

Hypothesis 3. LMX will mediate the relationship between supervisorsubordinate directional age differences and employee feedback perceptions and reactions, such that (a) having a younger supervisor will predict lower LMX scores, which will subsequently predict (b) lower ratings of informational justice, (c) lower ratings of interpersonal justice, and, at least partially through those two types of justice, (d) lower employee feedback satisfaction, (e) lower employee 
perceptions of feedback utility, and (f) lower employee motivation following the feedback event. Please see Figure 3 for a visual representation of this hypothesis.

Hypothesis 4. LMX will moderate the relationships between supervisorsubordinate directional age differences and employee feedback perceptions and reactions, such that higher-quality LMX will mitigate the negative effects of having a younger supervisor on (a) informational justice perceptions, (b) interpersonal justice perceptions, (c) employee feedback satisfaction, (d) employee perceptions of feedback utility, and (e) employee motivation following the feedback event. Please see Figure 4 for a visual representation of this hypothesis. 


\section{CHAPTER VII.}

\section{Method}

\section{Participants and Procedure}

Data from a larger study relating employee feedback reactions and age were analyzed in order to examine the hypothesized relationships. These data were collected from 371 Chinese employees of a British subsidiary of an automotive company located in Shenzhen, China. The organization was established in 2002 and employs approximately 500 workers, most of whom are engineers or technicians. Every February, annual performance evaluations are conducted for each employee. Performance feedback is then provided by each employee's direct supervisor in a face-to-face meeting with the employee. The age of participants in the sample ranged from 20 to 58; the average age of the sample was 34.98 ( $S D=$ 7.24) years. In the current sample, $65.5 \%$ of participants were under the age of 40 , and the remaining $34.5 \%$ were at or over the age of 40 . Of the sample, $83 \%$ were male. Participants in the sample had an average of 13.4 total years of education, indicating that on average, participants in the sample had some college education. This is consistent with trends in the sampled organization, which employs workers at the high school graduate level as well as the associate's degree level. This is also somewhat consistent with education trends in Shenzhen in general. Shenzhen organizations employ a large number of migrant workers, indicating 
that many of the city's employees do not currently live in, or are not originally from, Shenzhen. Workers who tend to migrate toward Shenzhen have historically had limited education beyond their high school years (e.g., Mok, 2002).

Study participants were administered surveys at two points in time in order to control for response bias (Podsakoff, MacKenzie, Lee, \& Podsakoff, 2003) and to allow for the examination of the effects of the study variables on the formation of employee feedback reactions over time. Time-1 surveys were administered 3 weeks after the annual performance feedback event, while Time- 2 surveys were administered an additional 5 weeks later. For both waves of surveys, participants were allowed to complete the surveys privately in a conference room at the worksite during work hours. These surveys were originally constructed in English, then translated into Chinese and back-translated into English to check translation accuracy. Surveys in both languages were offered to participants. When participant responses were in Chinese, a research associate in China translated these responses back into English. Participants were assured that their managers and other organizational representatives would not see their individual responses. The study announcement, along with a letter assuring confidentiality and the voluntary nature of participation, was distributed by the Human Resource Department to all employees except those working in the Human Resource Department itself. Excluding the HR Department employees reduced the $\mathrm{N}$ of 
potential respondents to 489 . Three hundred and eighty-two $(78.12 \%)$ employees responded to the Time-1 survey. Of the Time-1 participants, $371(97.12 \%)$ also responded to the Time-2 survey. These high response rates were likely achieved as a result of corporate sponsorship and provision of paid work time to complete the surveys. Only respondents who completed both waves of surveys were included in the final analyses.

\section{Measures}

Participants were asked to provide demographic information about both themselves and their immediate supervisors. The demographic variables assessed were: own age and gender; the gender and age of their immediate supervisor; whether the supervisor in question was older or younger than themselves; and how many years the respondents had been working with their immediate supervisors.

The Time-1 survey also included an LMX measure, as well as measures of participant perceptions of their experience during the feedback event (i.e., their perceptions of feedback valence and the informational and interpersonal justice they received). Participants had been given formal performance feedback 3 weeks prior to the Time-1 survey. The Time-2 survey measured participant reactions (i.e., their satisfaction, utility perceptions, and post-feedback work motivation) to the performance feedback they had received from their supervisor. At the time of 
the Time- 2 survey, the formal performance feedback had occurred about 8 weeks prior. Participants were asked to consider, when responding to both surveys, the most recent performance appraisal administered to them by their current supervisor.

Supervisor-subordinate directional age differences. Supervisorsubordinate directional age differences were calculated by subtracting (employee reported) supervisor age from employee age. Negative values resulted when the supervisor was older (i.e., the demographically normative situation); positive values resulted when the supervisor was younger than the employee (a nonnormative situation). Previous research has utilized similar methods to examine supervisor-subordinate age differences (e.g., Epitropaki \& Martin, 1999; Green, Anderson, \& Shivers, 1996; Vecchio, 1993). Study participants were also explicitly asked whether they perceived their supervisor as being older or younger than themselves; these responses were coded as $0=$ supervisor is relatively younger, 1 = supervisor is relatively older. These coded responses were included in initial analyses as well, as a measure of perceived supervisor-subordinate age patterns.

Leader-member exchange (LMX). The quality of the supervisor-subordinate dyadic relationship was measured at Time-1 using eight items from the LMX-8 scale developed by Bauer and Green (1996). These eight items were scored on a 
seven-point Likert-type scale (strongly disagree $=1$, strongly agree $=7$ ) and were averaged; a higher score indicated a higher-quality dyadic relationship perception by the employee. An example item is "My supervisor understands my problems and needs". Cronbach's alpha for this scale was 0.87. A complete list of these items can be seen in Appendix A.

Informational justice. Perceived informational justice regarding the feedback event was assessed at Time-1 using five items adapted from Colquitt's (2001) organizational justice scale. Participants were asked to indicate the quality of the information they received during the feedback event on a seven-point Likert-type scale ranging from "strongly disagree $=1$ " to "strongly agree $=7$ ". An example item is, "My supervisor explained the feedback thoroughly"; the complete list of all of the justice items can be found in Appendix A. Responses to the five items were averaged together to create one informational justice score, with higher scores indicating higher perceived justice. Cronbach's alpha for these items was 0.90 .

Interpersonal justice. Perceptions of interpersonal justice were assessed at Time-1 using four items adapted from Colquitt (2001). Participants were asked to indicate the quality of the interpersonal treatment they received from their supervisors during the feedback event on a seven-point Likert-type scale ranging from "strongly disagree $=1$ " to "strongly agree $=7$ ". An example item is, "My 
supervisor treated me in a polite manner during the feedback event”. Responses to the four items were averaged to create one interpersonal justice score, with higher scores indicating higher perceived justice. Cronbach's alpha for these items was 0.85 .

Satisfaction with feedback. Employee satisfaction with the feedback event was measured at Time-2 using 12 items adapted from the Satisfaction with Appraisal scale created by Dobbins et al. (1990). The items required participants to indicate how they felt about the feedback event on a seven-point Likert-type scale ( strongly disagree $=1$, strongly agree $=7$ ). An example item is, "I am satisfied with my most recent performance appraisal." Responses to the 12 items were averaged to create one satisfaction score, with higher scores indicating greater satisfaction with the feedback. Cronbach's alpha for these items was 0.96; a complete list of these items can be seen in Appendix A.

Perceptions of feedback utility. Employee utility perceptions were assessed at Time-2 using five items adapted from the Utility of Training scale developed by Ford and Noe (1987). Specifically, the scale items were adapted to measure perceptions of the utility of performance feedback instead of perceived utility of training. This measure asked participants to indicate how useful they found the feedback to be on a seven-point Likert-type scale (strongly disagree $=1$, strongly agree $=7$ ). An example item is, "The feedback I was given was useful for my 
development as an employee". Responses to the five items were averaged to create one perceived utility score, with higher scores indicating greater perceptions of feedback usefulness. Cronbach's alpha for these items was 0.97; a complete list of these items can be seen in Appendix A.

Work motivation following feedback. Employee post-feedback work motivation was measured at Time- 2 using four items from Bianchi and Ames (2008). These items required participants to indicate how the feedback they received impacted their motivation for performing their job duties. Participants were specifically asked in the survey instructions to attend to how the feedback impacted their motivation, and not to their absolute level of work motivation in general. An example item is, "My desire to work hard in this position"; the response options required participants to indicate how the feedback impacted this statement as well as the other three on a seven-point Likert-type scale ranging from "Very negative impact $=1$ " to "Very positive impact $=7$ ". Responses to the four items were averaged to create one motivation score, with higher scores indicating greater post-feedback work motivation. Cronbach's alpha for these items was 0.96; a complete list of these items can be found in Appendix A.

Exploratory construct feedback valence. Perceptions of feedback valence were assessed using two items used by Bianchi and Ames (2008). One of these items required participants to indicate their general impression of the feedback on 
a five-point scale ranging from "extremely negative $=1$ " to "extremely positive $=$ 5". The second item asked participants to indicate their overall judgment of the feedback event on a five-point scale ranging from "very bad $=1$ " to "very good = 5". The item responses were then averaged to create one valence score. The correlation between these two items was 0.93 . In exploratory analyses, this variable was included among the main predictors in the study models; for hypothesis testing, it was included among control variables.

Control variables. The relational demography literature indicates that some demographic factors besides chronological age can influence employee impressions and attitudes regarding their supervisors. One such factor is gender, with gender similarity in a dyad predicting greater interpersonal attraction and more positive employee attitudes (Geddes \& Konrad, 2003; Tsui et al., 1992; Tsui \& O'Reilly, 1989). Additionally, dyad tenure, or the length of time a supervisor and subordinate have been working together, is likely to influence employee attitudes toward the supervisor as well (Duarte et al., 1994; Epitropaki \& Martin, 1999; Schyns et al., 2005). As this study aimed to focus on the independent effects of supervisor-subordinate directional age differences on employee perceptions of and reactions to formal performance feedback, these other dyadic demographic elements were used as control constructs in the analyses. 


\section{CHAPTER VIII.}

\section{Results}

\section{Factor Analyses}

To ensure the construct validity of each of the model variables, several confirmatory factor analyses were conducted for comparison. This was especially important in the current study as many of the study outcomes were highly correlated and thus could potentially load onto the same latent variables. A sevenfactor model of these variables (i.e., feedback valence, LMX, informational justice perceptions, interpersonal justice perceptions, employee feedback satisfaction, employee perceptions of feedback utility, and employee motivation

following the feedback event) yielded the best fit, $\chi^{2}(719)=1721.11, p<0.05$, $\mathrm{CFI}=0.93, \mathrm{RMSEA}=0.06$. In this model all scale items loaded significantly onto their respective latent constructs, with standardized factor loadings ranging from 0.44 to 0.95 . Further, most of the standardized factor loadings were over 0.60 , i.e., well above the standard rule-of-thumb of .40 .

This model was compared with several alternative models to explore whether combining constructs would yield better fit. The first set of alternative models explored the possibility that each pair of the endogenous variables (valence, LMX, informational justice, and interpersonal justice) might converge into a single construct. This analysis involved computing six, six-factor models. All of 
these models yielded results significantly worse fitting than those for the sevenfactor model in which each endogenous variable was considered unique.

The second set of alternative models explored the possibility that any of the three outcome variables (employee feedback satisfaction, employee perceptions of utility, and employee post-feedback motivation) might be combined to form one construct. This part of the analysis contained three, six-factor models. Again, all of those alternative models yielded worse fit than the predicted seven-factor model. The results of these confirmatory factor analyses suggested that, despite being highly correlated, the scales assessing important study variables represented distinctive constructs. All subsequent analyses were conducted using this framework.

\section{Preliminary Analyses}

Following the establishment of the factor structure in the model, analyses were conducted in order to compute descriptive and inferential statistics for the study variables. These analyses revealed that, on average, participants in the sample reported receiving relatively positive (i.e., above the scale mean) feedback during their most recent performance appraisal event $(M=3.79, S D=0.75)$. Participants also generally had positive reactions to the feedback they received. Employee satisfaction $(M=5.24, S D=1.21)$, perceptions of feedback utility $(M=$ $5.46, S D=1.42)$ and post-feedback motivation $(M=5.55, S D=1.25)$ were all 
skewed, on average, toward the positive. Participants also generally reported above-mean LMX with their supervisors, $M=5.18, S D=1.06$. Their perceptions of the informational $(M=5.51, S D=1.18)$ and interpersonal justice $(M=5.58$, $S D=1.09)$ during their performance reviews were also relatively high.

Both supervisor-subordinate directional age differences - calculated by subtracting supervisor age from employee age — and employee-perceived supervisor-subordinate relative age $(M=0.71, S D=0.45)$ were also considered in the preliminary analyses. These two variables correlated at $-0.76(p<0.01)$. (Please see Table 1 for all variable correlations and their corresponding significance.) Due to this high correlation and the additional variance offered by its continuous rather than categorical nature, it was determined that supervisorsubordinate directional age differences would exclusively be used in subsequent analyses, following methods used by Epitropaki and Martin (1999), Green and colleagues (1996), and Vecchio (1993). The computed supervisor-subordinate directional age differences yielded a mean of $-4.12(S D=8.11)$, indicating that on average, participants tended to have slightly older supervisors than themselves. This variable ranged from -26 to 23 . Roughly $28.6 \%$ of the sample had supervisors younger than themselves. Employees tended to be about four years younger $(M=34.98, S D=7.24)$ than their supervisors on average $(M=39.10, S D$ $=5.00)$. 
In line with previous research (e.g., Brett \& Atwater, 2001; Colquitt et al., 2001; Levy \& Williams, 2004; Scandura, 1999; Wayne et al., 1997) LMX, feedback valence, informational justice, interpersonal justice, and all three feedback reactions were strongly correlated with one another. Supervisorsubordinate directional age differences correlated significantly and negatively with all three feedback reaction types, such that employees with younger supervisors tended to also have lower ratings of satisfaction $(r=-0.15, p<0.01)$, feedback utility $(r=-0.22, p<0.01)$, and post-feedback motivation $(r=-0.13, p<$ 0.05), providing preliminary support for Hypothesis 1. Supervisor-subordinate directional age differences also correlated significantly and positively with supervisor-subordinate dyad tenure $(r=0.22, p<0.01)$, indicating that employees with older supervisors tended to have worked with those supervisors for a longer period of time. Dyad tenure, in turn, correlated significantly and positively with employee age $(r=0.40, p<0.01)$ and supervisor age $(r=0.22, p<0.01)$. Dyadic age differences did not significantly correlate with informational justice $(r=0.02$, $p>0.05)$, interpersonal justice $(r=-0.06, p>0.05)$, or $\operatorname{LMX}(r=-0.07, p>$ $0.05)$.

\section{Structural Equation Models}

Structural Equation Model (SEM) analyses were conducted in order to test the study hypotheses. Separate analyses were conducted to assess each hypothesis. 
Note that the same pathway may have occurred in multiple models (e.g., supervisor-subordinate age differences predicting informational justice), but the results for that same pathway were likely to differ across models because all components of a given model are computed at once. Following the separate hypothesis analyses, one model incorporating all the hypotheses was conducted to test their potential unified validity. The results of each of these analyses are presented below.

Hypothesis 1 model. A path analysis was conducted that assessed the direct effects of supervisor-subordinate directional age differences on employee feedback satisfaction, employee feedback utility perceptions, and employee postfeedback motivation.

Main effects of directional age differences. This analysis revealed support for Hypothesis 1 , with dyadic age differences predicting satisfaction $(B=-0.02, \beta=$ $\left.-0.10, p<0.05, R^{2}=0.09\right)$, utility perceptions $\left(B=-0.03, \beta=-0.18, p<0.01, R^{2}=\right.$ $0.20)$, and post-feedback motivation $\left(B=-0.02, \beta=-0.10, p<0.05, R^{2}=0.06\right)$ in the expected direction, such that employees with younger supervisors had lower satisfaction, perceived utility, and post-feedback work motivation.

Control variables. This analysis accounted for the control variables and their impact on employee feedback reactions as well. Supervisor-subordinate dyad tenure did not significantly predict satisfaction $(B=0.00, \beta=0.00, p>0.05)$, 
utility perceptions $(B=0.00, \beta=0.00, p>0.05)$, or motivation $(B=0.00, \beta=$ $0.00, p>0.05)$. Supervisor-subordinate gender congruence also did not predict any of those outcomes $(B=-0.24, \beta=-0.08, p>0.05 ; B=-0.25, \beta=-0.07, p>$ 0.05 ; and $B=-0.19, \beta=-0.06, p>0.05$, respectively). However, feedback valence strongly predicted employee satisfaction $(B=0.86, \beta=0.54, p<0.05)$, utility perceptions $(B=0.79, \beta=0.42, p<0.05)$, and post-feedback motivation $(B=$ $0.68, \beta=0.41, p<0.05)$. Please see Table 2 for all analysis coefficients and their corresponding significance. See Figure 5 for the unstandardized and standardized path coefficients of this analysis.

Hypothesis 2 model. A second path analysis was conducted assessing both the direct effect of supervisor-subordinate directional age differences, and the (at least partial) mediating effects of informational and interpersonal justice, on the three types of employee feedback reactions.

Mediating effects of justice. Supervisor-subordinate age differences were not found to significantly predict informational $(B=0.08, \beta=0.06, p>0.05)$ or interpersonal $(B=-0.01, \beta=-0.04, p>0.05)$ justice, providing no support for the mediation effect proposed in Hypothesis 2.

Informational justice was found to significantly predict employee feedback satisfaction, $B=0.14, \beta=0.13, p<0.05$, but not employee utility perceptions $(B$ $=0.09, \beta=0.07, p>0.05)$ or employee post-feedback motivation $(B=0.07, \beta=$ 
$0.07, p>0.05)$. Interpersonal justice significantly predicted all three reaction types $(B=0.24, \beta=0.21, p<0.05 ; B=0.24, \beta=0.18, p<0.05$; and $B=0.22, \beta=$ $0.19, p<0.05$, respectively).

Main effects of directional age differences. Supervisor-subordinate directional age differences were found to predict employee feedback satisfaction $(B=-0.02, \beta=-0.10, p<0.05)$ and employee perceptions of feedback utility $(B=$ $-0.03, \beta=-0.17, p<0.05)$ in the expected direction, but did not significantly predict employee post-feedback motivation $(B=-0.01, \beta=-0.09, p>0.05)$ in this analysis.

Control variables. This analysis also accounted for the control variables and their impact on employee feedback reactions and justice perceptions. Supervisorsubordinate dyad tenure was not significant in predicting employee satisfaction $(B$ $=0.00, \beta=0.00, p>0.05)$, utility perceptions $(B=0.00, \beta=0.00, p>0.05)$, postfeedback motivation $(B=0.00, \beta=0.00, p>0.05)$, or perceptions of informational $(B=0.00, \beta=0.00, p>0.05)$ and interpersonal $(B=0.00, \beta=0.00$, $p>0.05)$ justice. Supervisor-subordinate gender congruence was not significant in predicting satisfaction $(B=-0.16, \beta=-0.05, p>0.05)$, utility perceptions $(B=-$ $0.17, \beta=-0.05, p>0.05)$, or post-feedback motivation $(B=-0.12, \beta=-0.04, p>$ 0.05), but it did significantly predict employee perceptions of informational justice $(B=-0.27, \beta=-0.10, p<0.05)$. Interpersonal justice was not significantly 
predicted by supervisor-subordinate gender congruence, $B=-0.16, \beta=-0.06, p>$ 0.05. Finally, feedback valence was strongly significant in predicting all three employee-feedback reaction types $(B=0.62, \beta=0.39, p<0.05 ; B=0.58, \beta=$ $0.31, p<0.05$; and $B=0.49, \beta=0.30, p<0.05$, respectively) as well as informational $(B=0.80, \beta=0.51, p<0.05)$ and interpersonal $(B=0.56, \beta=0.39$, $p<0.05)$ justice perceptions. Please see Figure 6 for the unstandardized and standardized path coefficients of this analysis.

Hypothesis 3 model. A third path analysis was conducted assessing the (at least partial) mediating effect of LMX in the relationships between supervisorsubordinate directional age differences and the three types of employee feedback reactions, as well as the (at least partial) mediating effect of LMX transferred through employee justice perceptions in these relationships.

Mediating effects of LMX and justice. Supervisor-subordinate age directional differences significantly predicted informational justice perceptions, $B=0.01, \beta=$ $0.08, p<0.05$, but in the opposite direction than was expected. Age differences, however, were not found to significantly predict interpersonal justice perceptions $(B=0.00, \beta=-0.01, p>0.05)$ or $\operatorname{LMX}(B=-0.01, \beta=-0.05, p>0.05)$.

Informational justice perceptions were not found to predict satisfaction $(B=$ $0.09, \beta=0.09, p>0.05)$, utility perceptions $(B=0.06, \beta=0.05, p>0.05)$, or motivation $(B=0.05, \beta=0.05, p>0.05)$, indicating that the significant 
relationship between supervisor-subordinate age differences and informational justice did not represent a mediating effect. Interpersonal justice, however, was found to significantly predict all three reaction-types $(B=0.18, \beta=0.16, p<$ $0.05 ; B=0.20, \beta=0.16, p<0.05$; and $B=0.19, \beta=0.17, p<0.05$, respectively). LMX significantly predicted both informational $(B=0.55, \beta=0.49, p<0.05)$ and interpersonal $(B=0.57, \beta=0.55, p<0.05)$ justice perceptions. While LMX did significantly predict employee satisfaction, $B=0.15, \beta=0.13, p<0.05$, it did not significantly predict employee utility perceptions $(B=0.10, \beta=0.08, p>0.05)$ or employee post-feedback motivation $(B=0.08, \beta=0.07, p>0.05)$. Overall, this SEM analysis provided no support for the meditational patterns proposed in Hypothesis 3.

Main effects of directional age differences. Supervisor-subordinate age differences were found to significantly predict employee satisfaction with the feedback, $B=-0.01, \beta=-0.10, p<0.05$, and employee perceptions of feedback utility, $B=-0.03, \beta=-0.17, p<0.05$, but did not significantly predict employee post-feedback motivation $(B=-0.01, \beta=-0.09, p>0.05)$ in this analysis.

Control variables. The analysis also included the control variables and their impact on employees' feedback reactions, justice perceptions, and LMX ratings. Supervisor-subordinate dyad tenure was not significant in predicting LMX $(B=$ $0.00, \beta=0.06, p>0.05)$, informational justice $(B=0.00, \beta=0.00, p>0.05)$, 
interpersonal justice $(B=0.00, \beta=0.03, p>0.05)$, employee satisfaction with feedback $(B=0.00, \beta=-0.01, p>0.05)$, employee perceptions of feedback utility $(B=0.00, \beta=-0.06, p>0.05)$, or employee post-feedback motivation $(B=0.00$, $\beta=0.00, p>0.05)$. Supervisor-subordinate gender congruence was also not significant in predicting $\operatorname{LMX}(B=-0.14, \beta=-0.05, p>0.05)$, informational justice $(B=-0.20, \beta=-0.07, p>0.05)$, interpersonal justice $(B=-0.14, \beta=-0.05$, $p>0.05)$, employee satisfaction $(B=-0.16, \beta=-0.05, p>0.05)$, employee utility perceptions $(B=-0.17, \beta=-0.05, p>0.05)$, or employee motivation $(B=-0.12, \beta$ $=-0.04, p>0.05)$. Finally, feedback valence was a strong positive predictor of $\operatorname{LMX}(B=0.54, \beta=0.38, p<0.05)$, informational justice $(B=0.50, \beta=0.49, p<$ $0.05)$, interpersonal justice $(B=0.25, \beta=0.55, p<0.05)$, employee satisfaction $(B=0.61, \beta=0.38, p<0.05)$, employee utility perceptions $(B=0.57, \beta=0.30, p$ $<0.05)$, and employee post-feedback motivation $(B=0.49, \beta=0.29, p<0.05)$. Please see Figure 7 for the unstandardized and standardized path coefficients of this analysis.

Hypothesis 4 model. A fourth path analysis was conducted assessing the moderating effect of LMX on the relationships between supervisor-subordinate directional age differences and employee satisfaction, employee utility perceptions, and employee post-feedback motivation, as well its moderating effect on informational and interpersonal justice. An interaction variable (cross-product) 
of the standardized LMX and age difference scores was calculated and inserted into the model to test the moderating effect of LMX.

Moderating effect of $\mathbf{L M X}$. LMX was not found to significantly moderate any of the proposed relationships. Specifically, the interaction between LMX and supervisor-subordinate age differences was not significant in predicting employee satisfaction $(B=-0.01, \beta=-0.01, p>0.05)$, employee utility perceptions $(B=$ $0.09, \beta=0.07, p>0.05)$, employee post-feedback motivation $(B=0.03, \beta=0.02$, $p>0.05)$, employee perceptions of informational justice $(B=0.03, \beta=0.03, p>$ $0.05)$, or employee perceptions of interpersonal justice $(B=-0.03, \beta=-0.03, p>$ 0.05). Thus, Hypothesis 4 was not supported.

Main effects of directional age differences and $L M X$. The individual variables comprising the interaction were tested in the model as endogenous variables as well. Supervisor-subordinate directional age differences were found to significantly predict employee satisfaction $(B=-0.01, \beta=-0.09, p<0.05)$ and employee utility perceptions $(B=-0.03, \beta=-0.18, p<0.05)$ in the expected direction. They were not found to significantly predict employee post-feedback motivation $(B=-0.01, \beta=-0.09, p>0.05)$, employee perceptions of informational justice $(B=0.01, \beta=0.08, p>0.05)$, or employee perceptions of interpersonal justice $(B=-0.00, \beta=-0.01, p>0.05)$. 
LMX was found to be a significant predictor of employee satisfaction $(B=$ $0.15, \beta=0.13, p<0.05)$, informational justice $(B=0.55, \beta=0.49, p<0.05)$, and interpersonal justice $(B=0.57, \beta=0.55, p<0.05)$, but not employee utility perceptions $(B=0.11, \beta=0.08, p>0.05)$, or employee post-feedback motivation $(B=0.08, \beta=0.07, p>0.05)$.

Control variables. The analysis also accounted for the control variables and their impact on employees' feedback reactions and justice perceptions. Supervisor-subordinate dyad tenure was not significant in predicting informational justice $(B=0.00, \beta=0.00, p>0.05)$, interpersonal justice $(B=$ $0.00, \beta=0.03, p>0.05)$, employee satisfaction with feedback $(B=0.00, \beta=$ $-0.01, p>0.05)$, employee perceptions of feedback utility $(B=0.00, \beta=-0.06, p$ $>0.05)$, or employee post-feedback motivation $(B=0.00, \beta=0.00, p>0.05)$. Supervisor-subordinate gender congruence was also not significant in predicting informational justice $(B=-0.09, \beta=-0.03, p>0.05)$, interpersonal justice $(B=-$ $0.19, \beta=-0.07, p>0.05)$, employee satisfaction $(B=-0.16, \beta=-0.05, p>0.05)$, employee utility perceptions $(B=-0.18, \beta=-0.05, p>0.05)$, or employee postfeedback motivation $(B=-0.13, \beta=-0.04, p>0.05)$. Finally, feedback valence was a strong positive predictor of informational justice $(B=0.51, \beta=0.32, p<$ 0.05), interpersonal justice $(B=0.25, \beta=0.17, p<0.05)$, employee satisfaction 
$(B=0.61, \beta=0.38, p<0.05)$, employee utility perceptions $(B=0.57, \beta=0.31, p$ $<0.05)$, and employee motivation $(B=0.49, \beta=0.29, p<0.05)$. Please see Figure 8 for the unstandardized and standardized path coefficients of this analysis.

Full model. To explore the validity of all study hypotheses occurring together, a full model incorporating both the moderating and mediating effects of LMX was explored. This model assessed the direct effect of supervisorsubordinate age differences on employee feedback reactions, as well as the mediating effects of justice perceptions and LMX. Further, this model assessed the moderating effect of LMX in the relationships between age differences and informational justice perceptions, interpersonal justice perceptions, employee satisfaction with feedback, employee perceptions of feedback utility, and employee motivation following the feedback event.

Hypothesis 1 in the full model. Supervisor-subordinate directional age differences were found to significantly predict employee feedback satisfaction, $B$ $=-0.01, \beta=-0.09, p<0.05$, and employee utility perceptions, $B=-0.03, \beta=-$ $0.18, p<0.05$, in the expected direction. However, age differences were not significant in predicting employee post-feedback motivation, $B=-0.01, \beta=-0.09$, $p>0.05$. Thus, Hypothesis 1 received partial support in the full model.

Hypothesis 2 in the full model. Supervisor-subordinate directional age differences were not found to significantly predict employee perceptions of 
informational $(B=0.01, \beta=0.08, p>0.05)$ or interpersonal $(B=0.00, \beta=-0.01$, $p>0.05)$ justice, providing no support for Hypothesis 2 in the full model. Informational justice, in turn, was not a significant predictor of employee satisfaction $(B=0.09, \beta=0.09, p>0.05)$, employee utility perceptions $(B=0.05$, $\beta=0.04, p>0.05)$, or employee post-feedback motivation $(B=0.05, \beta=0.04, p$ $>0.05)$. Interpersonal justice perceptions, however, were significant in positively predicting all three reaction-types $(B=0.18, \beta=0.16, p<0.05 ; B=0.21, \beta=$ $0.16, p<0.05$; and $B=0.20, \beta=0.17, p<0.05$, respectively).

Hypothesis 3 in the full model. Supervisor-subordinate directional age differences were not found to positively predict employee ratings of $\mathrm{LMX}, B=$ $-0.01, \beta=-0.05, p>0.05$, providing no support for Hypothesis 3 in the full model. LMX, in turn, did positively predict employee perceptions of informational $(B=0.55, \beta=0.49, p<0.05)$ and interpersonal $(B=0.57, \beta=0.55$, $p<0.05)$ justice, as well as employee satisfaction with the feedback event $(B=$ $0.15, \beta=0.13, p<0.05)$. However, LMX was not a positive predictor of employee perceptions of feedback utility, $B=0.11, \beta=0.08, p>0.05$, or employee motivation following the feedback event, $B=0.08, \beta=0.07, p>0.05$.

Hypothesis 4 in the full model. The interaction between LMX and supervisorsubordinate age differences was not found to significantly predict employee perceptions of informational $(B=0.03, \beta=0.03, p>0.05)$ or interpersonal $(B=-$ 
$0.03, \beta=-0.08, p>0.05)$ justice, nor was it found to predict employee satisfaction $(B=-0.01, \beta=-0.01, p>0.05)$, employee utility perceptions $(B=0.09, \beta=0.07$, $p>0.05)$, or employee post-feedback motivation $(B=0.03, \beta=0.02, p>0.05)$, indicating no moderating effect of LMX in the proposed relationships. Thus, Hypothesis 4 received no support in the full model.

Control variables in the full model. This analysis also accounted for the study control variables and their impact on employee justice perceptions, feedback reactions, and ratings of LMX. Supervisor-subordinate dyad tenure was not significant in predicting $\operatorname{LMX}(B=0.00, \beta=0.06, p>0.05)$, informational justice $(B=0.00, \beta=0.00, p>0.05)$, interpersonal justice $(B=0.00, \beta=0.03, p>0.05)$, employee satisfaction with feedback $(B=0.00, \beta=-0.01, \mathrm{p}>0.05)$, employee perceptions of feedback utility $(B=0.00, \beta=-0.07, p>0.05)$, or employee postfeedback motivation $(B=0.00, \beta=0.00, p>0.05)$. Supervisor-subordinate gender congruence was also not significant in predicting $\operatorname{LMX}(B=-0.14, \beta=-$ $0.05, p>0.05)$, informational justice $(B=-0.09, \beta=-0.03, p>0.05)$, interpersonal justice $(B=-0.19, \beta=-0.07, p>0.05)$, employee satisfaction $(B=-$ $0.16, \beta=-0.05, p>0.05)$, employee utility perceptions $(B=-0.18, \beta=-0.05, p>$ $0.05)$, or employee post-feedback motivation $(B=-0.13, \beta=-0.04, p>0.05)$. Finally, feedback valence was a strong positive predictor of $\operatorname{LMX}(B=0.54, \beta=$ 
$0.38, p<0.05)$, informational justice $(B=0.51, \beta=0.32, p<0.05)$, interpersonal justice $(B=0.25, \beta=0.17, p<0.05)$, employee satisfaction $(B=0.61, \beta=0.38, p$ $<0.05)$, employee utility perceptions $(B=0.59, \beta=0.31, p<0.05)$, and employee motivation $(B=0.49, \beta=0.29, p<0.05)$. Please see Figure 9 for the unstandardized and standardized path coefficients of this full model analysis.

\section{Exploratory Analyses of Feedback Valence Moderation}

While feedback valence was entered in the above models as a control variable in order to explore the isolated effect of supervisor-subordinate directional age differences on employee feedback perceptions and reactions, prior feedback research and the strong effect of valence on the study variables in this sample indicated that its role in the model might warrant further examination. As such, exploratory path models were constructed in which valence acted as a moderator of the relationships between the primary predictors and outcomes. When significant moderating effects were found, all visual graphs were constructed by calculating outcomes at one standard deviation above and below study variable means, following methods used by Cohen, Cohen, West, and Aiken (2003).

First, a path model was constructed exploring the moderating role of valence in the relationships between supervisor-subordinate directional age differences and the study variables of interest (LMX, informational justice perceptions, interpersonal justice perceptions, employee feedback satisfaction, employee 
perceptions of feedback utility, and employee motivation following the feedback event). This model revealed that the calculated interaction term comprised of (standardized) feedback valence and age difference scores was significant in predicting $\mathrm{LMX}, B=-0.13, \beta=-0.12, p<0.05$. The nature of this interaction was such that having a younger supervisor tended to reduce the effects of feedback valence on LMX ratings (i.e., with a younger supervisor, LMX was more or less equal regardless of negative or positive feedback valence). When the supervisor was relatively older than the employee, however, employees' LMX ratings were dependent on feedback valence, such that lower LMX was reported when feedback was negative, and higher LMX was reported when feedback was positive. The nature of this interaction can be seen in Figure 10.

Valence was also found to significantly moderate the relationships between supervisor-subordinate directional age differences and employee feedback satisfaction $(B=0.11, \beta=0.08, p<0.05)$ and employee post-feedback motivation $(B=0.13, \beta=0.09, p<0.05)$. The nature of these interactions was contrary to the moderating effect of valence in the relationship between dyadic age differences and LMX, mentioned above. Specifically, employees with older supervisors tended to have satisfaction reactions that were less dependent on feedback valence. However, employees with relatively younger supervisors tended to have satisfaction reactions that were more dependent on feedback valence, such that 
employees in this condition had particularly low satisfaction when feedback was negative and particularly high satisfaction when feedback was positive. A similar effect was seen with employee motivation, such that employees with older supervisors tended to have motivation scores that were less dependent on feedback valence, while employees with younger supervisors tended to have particularly low motivation scores when feedback was negative and particularly high motivation scores when feedback was positive. Please see Figures 11 and 12 for a visual representation of these relationships.

Valence was not found to moderate the relationships between supervisorsubordinate directional age differences and informational $(B=-0.09, \beta=-0.07, p$ $>0.05)$ or interpersonal $(B=-0.03, \beta=-0.02, p>0.05)$ justice perceptions, nor was it found to significantly moderate the relationship between dyadic age differences and employee perceptions of feedback utility $(B=0.11, \beta=0.07, p>$ $0.05)$.

Next, feedback valence was examined as a potential moderator in the relationships between LMX and the other primary outcome variables (informational justice perceptions, interpersonal justice perceptions, employee feedback satisfaction, employee utility perceptions, and employee post-feedback motivation). Valence was not found to significantly moderate any of these 
relationships, $p s>0.05$, indicating that the impact of LMX on these variables did not change with variations in feedback valence.

Finally, a series of models were tested in which feedback valence was examined as a potential moderator in the relationships between the two types of justice perceptions (informational and interpersonal) and the three feedback reaction outcomes (employee feedback satisfaction, utility perceptions, and motivation). Valence was not found to significantly moderate any of these relationships, $p \mathrm{~s}>0.05$, indicating that the effects of justice perceptions on feedback reactions did not change as a function of whether the feedback was overall negative or positive. 


\section{CHAPTER IX.}

\section{Discussion}

The results of the current study paint an interesting and telling picture of the impact of supervisor-subordinate directional age differences on employee feedback reactions and perceptions. Further, this research sheds light on these organizational relationships as they appear in a country that, much like the United States, is experiencing dramatic and rapid shifts in its workforce demographics. This study adds to existing feedback reactions literature by tying in dyadic directional age differences, considering the simultaneous effects of LMX and justice in forming feedback reactions, and studying these variables in the context of a largely understudied but extremely valuable economic market. The current study aids our understanding of supervisor-subordinate relative age in the changing workforce and how it impacts important individual and organizational outcomes, as well as aiding our understanding of organizational practices and norms in a country that both mirrors and opposes the United States in many ways.

\section{Hypothesized Relationships}

Direct effects of supervisor-subordinate directional age differences. The results of the SEM assessing Hypothesis 1 revealed that supervisor-subordinate directional age differences, or in practical terms, having a younger supervisor, negatively predicted employee satisfaction with feedback, perceptions of 
feedback utility, and motivation following the feedback event as hypothesized. However, the inclusion of mediating variables in subsequent analyses resulted in directional age differences significantly predicting only employee feedback satisfaction and utility perceptions. In these more comprehensive models, postfeedback motivation was not significantly predicted by whether supervisors were older or younger than their respective employees.

\section{Mediating effects of informational and interpersonal justice. The SEM}

analysis assessing Hypothesis 2 revealed that supervisor-subordinate directional age differences did not predict employee perceptions of informational or interpersonal justice as experienced during the feedback event. As such, no support was found for the hypothesized mediating effects of justice perceptions in the relationships between supervisor-subordinate directional age differences and the three types of employee feedback reactions measured. When LMX was included as a mediating variable in the model assessing Hypothesis 3, directional age differences actually did reveal a significant predictive relationship with informational justice perceptions. However, this effect was contrary to expectations; the path coefficients indicated that having a younger supervisor actually increased employee perceptions that they had been treated with fair informational justice during the feedback. This relationship was modest and became non-significant when LMX was included in the model as a moderating 
variable (i.e., in the analyses assessing Hypothesis 4 and the full model). Further, because informational justice perceptions were not found to significantly predict employee satisfaction, utility perceptions, or post-feedback motivation in the analysis for Hypothesis 3, the informational justice perceptions variable was ruled out as a mediator between directional age differences and any of the employee feedback reactions.

Theory and existing research suggests that employee perceptions of the justice they experience during a feedback event should predict their reactions to that event (Cropanzano et al., 2002; Erdogan, 2002; Greenberg, 1986; 1990; Van den Bos et al., 2001). In the current study, this was the case for interpersonal justice perceptions, but not for informational justice perceptions. In the SEM analysis assessing Hypothesis 2, it was found that informational justice significantly predicted employee satisfaction with the feedback event, but in subsequent analyses this effect disappeared. By contrast, perceptions of interpersonal justice remained significant in predicting employee satisfaction, utility perceptions, and post-feedback motivation throughout all of the analyses examining these relationships. However, the previously mentioned lack of relationship between supervisor-subordinate directional age differences and either type of justice indicated that informational and interpersonal justice perceptions were not mediators in the directional age-employee feedback reactions relationships. 
Mediating effect of LMX. The SEM analysis for Hypothesis 3 indicated that LMX was not a mediator between supervisor-subordinate directional age differences and informational or interpersonal justice perceptions; nor was it a mediator between age differences and any of the three employee feedback reactions measured. Supervisor-subordinate directional age differences were not found to significantly predict LMX scores at all.

LMX scores, however, did strongly predict informational and interpersonal justice perceptions. This strong relationship is not surprising; research has indicated that these constructs are highly related to one another (Leow \& Kuong, 2009; Masterson et al., 2000). Moreover, social exchange theory posits that each of these constructs predict positive outcomes in the same way (Cropanzano et al., 2002; Emerson, 1976; Wayne et al., 1997). However, it is important to note that data assessing LMX and employee justice perceptions were collected crosssectionally. The current research cannot truly provide a causal path between these closely related variables. Thus, it is possible that perceiving better justice coming from the supervisor caused employees to also perceive a better relationship with that supervisor, as suggested by Masterson and colleagues (2000).

The SEM analyses for Hypothesis 3 accounted for the possibility of a direct relationship between LMX and the three types of employee feedback reactions as well. LMX was found to significantly predict employee satisfaction with the 
feedback event, but not employee perceptions of feedback usefulness or employee motivation following the feedback. These effects (or lack thereof) persisted in the analysis incorporating the full model.

Moderating effect of LMX. The SEM analysis assessing Hypothesis 4 found that a cross-product between directional age differences and LMX scores was not significant in predicting employee informational and interpersonal justice perceptions, or any of the three types of feedback reactions measured. The SEM analysis assessing the full model simultaneously revealed the same results. As such, LMX was not found to moderate any of the relationships between supervisor-subordinate age differences and the study variables, indicating that dyadic directional age differences generally had the same impact on employee perceptions of and reactions to the feedback event regardless of the quality of the relationship in the dyad.

Theoretical explanations of the study results. The results of the current study suggest that having a younger supervisor does predict poorer employee reactions to a supervisor-led performance feedback event over time, but that these poorer reactions do not occur because of perceptions of reduced interactional justice or LMX in these relationships. Initial analyses suggested that employee feedback satisfaction, employee perceptions of feedback usefulness, and employee motivation following the feedback event were all negatively affected, 
but subsequent analyses suggested that post-feedback motivation was not among the employee reactions negatively affected by having a younger supervisor.

This may be an artifact of general motivational differences in China as compared to the United States. Previous research has shown that Chinese employees, when asked about their work preferences, tend to cite good wages, good working conditions, and loyalty from the organization as being highly motivating factors (Fisher \& Yuan, 1998). These motivating factors tend to focus more highly on aspects of the organization rather than the supervisor. Further, given the notion that Chinese citizens tend to be more collectivist and grouporiented (Child \& Markoczy, 1993; Hofstede, 2001), it may be that Chinese employees are more motivated to perform well in their jobs for the benefit of the entire organization rather than for their supervisor or themselves. It is possible that work motivation, itself, is a group-focused phenomenon in China and is less susceptible to attributes of any particular supervisor or singular event.

In this study, I hypothesized that employee perceptions of informational justice, interpersonal justice, and LMX would mediate the relationships between supervisor-subordinate directional age differences and the various types of employee feedback reactions; these hypotheses were not supported. Directional age differences were not a significant predictor of any of those employee perception variables. Again, these findings should be interpreted in the context of 
the cultural values of the nation in which the data were collected. In China, one such value, high power distance (also referred to as respect for hierarchy; Hofstede, 2001), indicates that employees should and will be respectful toward their supervisors regardless of any demographic or other attributes. While feedback reaction survey items asked participants to indicate how they felt about the feedback event, these justice and relational perception variables were more focused on rating the behaviors of the supervisor, generally and in the context of the feedback event. As such, strong traditional Chinese values may have masked any effects of perceived career timetable violations (e.g., Lawrence, 1984). Future research would greatly benefit from further examination of these relationships as they occur in regions with strong cultural values and norms.

Although the hypothesized mediators were not significant in the current study, future researchers may continue efforts to understand the effects of supervisorsubordinate directional age differences through the examination of other possible mediators. First, while it was theoretically assumed in the current sample that implicit career timetables and their effects would play a role in the supervisorsubordinate relationship, this was not explicitly measured. Some measurement of this variable may have clarified the role of implicit career timetables in supervisor-subordinate demographic patterns and their subsequent effects. Other studies have explicitly measured these timetables by calculating the difference 
between people's conceptualizations of the "typical" age for someone in their career level and their own age (Lawrence, 1984), and by examining people's conceptualizations of the "typical" age for someone in other career stages (for instance, that of a manager) (Lawrence, 1990).

Conversely, it may be that the outcomes in the current study (employee feedback reactions) are actually mediators in a much more practically important relationship. Performance feedback is delivered with the ultimate aim of having a positive impact on employee performance (Brett \& Atwater, 2001; Kluger \& DeNisi, 1996). This research exclusively examined feedback reactions. Past researchers have studied similar constructs (e.g., Bianchi \& Ames, 2008; Brett \& Atwater, 2001) with the idea that these reactions and perceptions are precursors to employee behavior (i.e., an employee who is more satisfied with feedback, finds it more useful, and feels more motivated by it will be more likely to improve his or her performance). However, with the results of the current study it is impossible to tell whether this is truly the case. Future research may benefit from the exploration of the effect of having a relatively younger supervisor on employee performance in general, rather than on feedback reactions exclusively. Empirical and theoretical evidence suggests that this effect will be negative (Lawrence, 1984; Perry et al., 1999; Shore et al., 2003); future researchers may examine 
whether performance feedback episode characteristics (and subsequent employee reactions) play a mediating role.

LMX was not only hypothesized as a mediator in the current study, but also as a moderator expected to alleviate the negative effects of having a younger supervisor on employee perceptions of and reactions to performance feedback. This was not supported; none of those relationships varied as a function of variations in LMX. As such, study participants were negatively impacted in terms of their feedback satisfaction and utility perceptions when they had relatively younger supervisors regardless of whether or not they had built a positive relationship with those supervisors.

This finding is troubling, considering that the study results as well as existing research (e.g., Collins et al., 2009; Geddes \& Konrad, 2003; Lawrence, 1984; 1990; Perry et al., 1999; Tsui et al., 2002) suggest that there are possible negative outcomes for employees who are paired with relatively younger supervisors, and the incidence of these demographically non-normative pairings are increasing (Liebold \& Voelpel, 2006; Mor Barak, 2011). Consequently, researchers should build on the current study by further examining moderators that may reduce the negative impact on employees.

One such construct that is receiving increasing attention in recent organizational research is the idea of organizational climate. Organizational 
climate refers to shared perceptions among employees regarding some aspect of organizational functioning. Climate perceptions can be assessed by observing and examining the way organizations deal with their members and the environment, through policies and practices as well as through more informal interactions with supervisors and coworkers (James \& Jones, 1974). At the individual level, climate takes the form of "a set of attitudes and expectancies which describe the organization in terms of both static characteristics (i.e., degree of autonomy) and behavior-outcome contingencies" (Campbell, Dunnette, Lawler, \& Weick, 1970, pg. 390).

Researchers have suggested that organizational climate can have multiple dimensions. Research along these lines has focused on the climate of some specific aspect of organizational functioning, such as shared perceptions regarding individual autonomy, reward orientation, consideration and support (James \& Jones, 1974), or safety (Zohar, 2000). Justice climate, for instance, refers to shared, organization- or team-level perceptions regarding the importance and emphasis placed on justice within an organization (Liao \& Rupp, 2005). More positive perceptions of justice climate have been linked to individual helping behaviors, even when individual perceptions of justice were controlled for (Naumann \& Bennett, 2000). Justice climate can focus on either the organization or the supervisor as a source of justice, and both of these are related to a number 
of important work outcomes such as supervisor- and organization-directed commitment, individual satisfaction, and extra-role performance (Liao \& Rupp, 2005).

Justice climate within the particular organization sampled in the current study could have impacted employee justice perceptions regarding their performance appraisals. If employees felt that their supervisor placed a strong importance on justice but did not behave in a just way within the context of the feedback event, this could have been even more detrimental for employee feedback reactions. By contrast, if employees felt their supervisor or organization did not value justice, their justice perceptions may have been less impactful on their reactions. Past research findings indicate that Chinese organizations in general may have a lower justice climate due to the nation's value of power distance (Brockner et al., 2001); current research findings indicate that informational justice in particular may be less of a priority. Nevertheless, future researchers should examine justice climate as a potential moderator in various cultures and contexts in order to widen our understanding of how employee feedback reactions are formed.

Another aspect of organizational climate that may be particularly helpful in aiding our understanding of the impact of having a younger or older supervisor is age-diversity climate. Diversity climate in general refers to shared perceptions among workers regarding the value the organization places on diversity, which 
can again be assessed through perceptions of policies and informal interactions at work (Hicks-Clarke \& Iles, 2000). Age diversity climate in particular, referring to shared perceptions regarding the degree to which the organization values employing and retaining employees of different ages, could have a strong impact on how employees view supervisor-subordinate age differences.

Relative to the current study, if the sampled organization promotes and values a variety of age-difference patterns, then the occurrence of having a younger supervisor may have been more likely to be viewed by employees as a positive thing, resulting in better-than-expected feedback reactions. Conversely, a low age diversity climate would predict the negative attitudes towards a relatively younger manager that we would expect to see. Supporting that possibility, one study found that increased diversity in terms of race and age predicted decreased team performance, possibly due to a relatively low diversity climate in which such differences were not encouraged (Timmerman, 2000). Future researchers should account for age diversity climate when considering how supervisor-subordinate age differences in general and directional age differences in particular impact employee attitudes and behaviors.

Although the study hypotheses were focused on the direct and indirect effects of supervisor-subordinate directional age differences, the path analyses assessing the hypotheses revealed interesting direct relationships between LMX, employee 
justice perceptions, and the various employee feedback reactions that were measured. First, there were differences in the two types of justice perceptions in terms of their ability to predict employee feedback reactions. Overall, interpersonal justice was a strong predictor of all three reaction types throughout the analyses. Conversely, informational justice tended not to predict any of the three reactions, with the exception of its effect on employee feedback satisfaction in initial analyses. Feedback reactions for the study participants seemed more dependent on how they were treated by their supervisor rather than the quality and extent of the information and content they received during the feedback event.

Organizational justice research conducted in Western cultures indicates that both interpersonal and informational justice should predict employee feedback reactions (Cropanzano et al., 2002; Erdogan, 2002; Greenberg, 1986), as justice in general and interactional justice in particular tends to predict positive organization-focused and supervisor-focused attitudes and behaviors (Colquitt et al., 2001; Greenberg, 1990). However, Chinese organizational research suggests that informational justice may not be as valued in Eastern cultures. For instance, research has shown that while relationships and hierarchy are important in Chinese employee work motivation, "being in on things" or receiving explanations for organizational behavior is not at all important (Fisher \& Yuan, 1998). Tata and colleagues (2003) found in their cross-cultural comparison of 
performance evaluation fairness ratings that Chinese employees tended to be more concerned with social sensitivity during the feedback event, while U. S.

employees tended to be more concerned with "account giving", or hearing adequate explanations for their performance ratings. The researchers suggested these effects may be due to the high power distance in Chinese organizations which may cause employees to be less likely to expect these kinds of explanations from their supervisors.

Relevant regional differences exist as well; one study found that employees in the People's Republic of China (PRC) tended to desire open, honest, and direct communications from their supervisors, while this was less important to employees in the Hong Kong region (Chow, 1995). Although these findings were published prior to major political changes in the Hong Kong region, cultural researchers have suggested that value and norm differences between this region and the PRC persist (e.g., Dessler \& Tan, 2006). While Shenzhen, where the current study was conducted, is technically part of the PRC, its close proximity to Hong Kong allows the two regions to have close business, trade, and social links and thus it is likely that Hong Kong values and norms transfer over to Shenzhen organizational functioning. Hong Kong has also been described as a region with extremely low uncertainty avoidance values, further suggesting that employees within this society do not have a strong need to know exact reasons and 
justifications for organizational procedures and outcomes (Chow, 1995; Hofstede, 2001).

Throughout China the cultural value of power distance remains relatively high. Research has shown that employees in cultures valuing power distance tend to exhibit fewer negative effects as a result of not being given a "voice" in a variety of workplace situations (Brockner et al., 2001). While voice is a procedural justice element that does not necessary fall under the purview of informational justice (Brockner et al., 2001), these findings suggest that certain elements of justice in general may have less of an impact in companies where power distance is high and thus inclusive treatment coming from the top down is less of an expectation.

Employee ratings of LMX directly predicted employee feedback satisfaction, but not perceptions of feedback usefulness or post-feedback motivation. Again, while LMX is a supervisor-focused construct, the three types of feedback reactions measured in the current study represented feedback event-focused constructs. These relationships (or lack thereof) suggest that the two are distinct for Chinese employees. Put another way, employees perceived feedback as being useful (or not useful) and motivating (or not motivating) regardless of whether or not they perceived a good relationship with their supervisor. 
Research has indicated that many Chinese employees tend to exhibit good contextual performance at work regardless of how they perceive their relationship with their supervisor, due to the collectivist nature of the traditional Chinese (Hui et al., 2004). As such, it may be that the employees in the current sample remained committed and motivated to use the feedback to improve their own performance because their desire to contribute positively to the organization transcended any relational issues with their supervisors. However, this commitment may not have reflected how they felt about the feedback event. LMX did significantly predict the degree to which feedback was satisfying for employees, with employees perceiving higher LMX reporting greater satisfaction with the feedback event. Multi-dimensional measures of LMX certainly indicate an affective component (Liden \& Maslyn, 1998; Scandura \& Graen, 1984; Schreisheim et al., 1999), among other dimensions such as loyalty, mutual respect, and trust (Hui et al., 2004; Liden \& Maslyn, 1998). As such, it appears that these affective attributes of LMX tend to also predict affective attributes of other supervisor-subordinate interactions.

Despite the lack of significant relationships between LMX and some of the other employee feedback reactions in the current study, some research has shown that LMX predicts task and contextual performance among Chinese employees (Perry et al., 1999; Tsui et al., 2002; Wang, Law, Hackett, Wang, \& Chen, 2005). 
While LMX may not have predicted many outcomes in this particular sample, this suggests that its inclusion in studies of Chinese supervisor-subordinate dyad functioning is still essential as it predicts important workplace outcomes.

Summary of hypothesized results. The current results revealed some unexpected findings, but these findings must be interpreted within the context of the study. While supervisor-subordinate directional age differences did not universally predict reductions in the study variables, they did predict reduced employee reactions when the participants were asked to rate a feedback event. When participants were asked to describe attributes or behaviors of their supervisor, this effect did not appear, possibly due to strong values regarding power distance and respect for hierarchy inherent in traditional Chinese culture. Because directional age differences were not related to these more supervisordirected variables the hypothesized mediating and moderating effects were not supported. However, future research can build on these findings by examining other potential mechanisms for the effects of supervisor-subordinate directional age differences on employee feedback reactions.

\section{Exploratory Relationships}

Research suggests that feedback valence, or the degree to which performance feedback is overall positive or negative, strongly determines how employees will perceive and react to a performance feedback event (Brett \& Atwater, 2001; 
Kluger \& DeNisi, 1996; Mabe \& West, 1982; Shrauger, 1975). In the current study, valence as a control variable consistently predicted other study variables of interest (informational justice perceptions, interpersonal justice perceptions, employee satisfaction with the feedback event, employee perceptions of feedback usefulness, and employee motivation following the feedback event). As such, an exploratory examination of valence was conducted in which the moderating effects of valence on the relationships between the study variables were considered.

These exploratory analyses revealed that feedback valence moderated the relationship between directional age differences and LMX, such that employees perceived roughly similar levels of LMX with their relatively younger supervisors regardless of valence. Employees with relatively older supervisors, however, perceived greater variations in LMX as a result of differences in feedback valence. Put another way, when employees' supervisors were relatively older (a demographically "normal” situation), being given negative feedback led to lower LMX scores, while being given positive feedback led to higher LMX scores. This valence discrepancy did not matter as much when supervisors were relatively younger.

This was reversed in the significant moderating effect of valence on the relationships between supervisor-subordinate directional age differences and 
employee feedback satisfaction and post-feedback motivation. In these

relationships, employees with relatively younger supervisors (a demographically

"non-normal" situation) tended to have satisfaction and motivational reactions

that were more dependent on feedback valence. Older supervisors, by contrast, tended to garner employee feedback reactions that were less dependent on variations in feedback valence.

These exploratory results carry with them several interesting implications.

First, it is notable that the moderating effect of valence on the relationship between dyadic age differences and LMX is the exact opposite of the moderating effect of valence on the relationships between age differences and employee feedback satisfaction and motivation. When LMX was the outcome, older supervisors garnered more extreme reactions based on variations in feedback valence. Given strong age-related reverence norms in China coupled with the values of high power distance and respect for hierarchy (Brockner et al., 2001; Chow, 1995; Tata et al., 2003), it is logical that Chinese employees would perceive negative feedback as a failure on their own part in upholding the supervisor-subordinate relationship. Conversely, positive feedback would be perceived as a success on the part of the employee, and thus their end of the supervisor-subordinate relationship would be upheld and rated positively. Younger supervisors, however, would not be viewed through the same age-related 
reverence values and thus relatively older employees would not be as concerned with their own success or failure in upholding the relationship.

While LMX was measured multi-dimensionally in the current study, it was not measured in a way that would allow for the exploration of whether LMX scores depended on how the employees felt about their supervisors, or on how the employees perceived their supervisors felt about them (likely these scores reflected a combination of those two viewpoints). However, the emphasis placed on relationships and respect for hierarchy in Chinese organizations (Child \& Markoczy, 1993) likely played a role in the valence-based LMX variations seen here.

The reverse was true for the relationship between supervisor-subordinate directional age differences and employee reactions; here, relatively younger supervisors garnered higher satisfaction and motivation scores when delivering positive feedback and lower satisfaction and motivation scores when delivering negative feedback. Of particular relevance here may be contrast effect (Hovland, Harvey, \& Sherif, 1957; Sherif \& Hovland, 1961). Contrast effect occurs because context tends to affect how people view a target (Herr, Sherman, \& Fazio, 1983). To explore how contrast effect operates, one might consider its opposite, the assimilation effect (Herr et al., 1983). If an individual is primed with many negative words and then read a description of a person, they may begin to think of 
that described person negatively. However, if an individual is primed with extremely negative words and then they read a description of a person, they may begin to view that person more positively, as a contrast to how they have been primed. Contrast effect has been successfully studied and found in terms of word lists (e.g., Herr et al., 1983; Sherman, Ahlm, Berman, \& Lynn, 1987) and comparisons of physical attractiveness (Wedell, Parducci, \& Gieselman, 1987).

In the current study, employees may have been "primed" by age-related cultural values to expect less from their younger supervisors. As such, when those younger supervisors delivered positive feedback, employees were pleasantly surprised and thus were more satisfied and more motivated in their work. Younger supervisors delivering negative feedback, however, may have garnered especially low satisfaction and motivation scores because employees were already reacting negatively to having a younger supervisor and negative feedback amplified those reactions.

While employee reactions differed as expected according to variations in valence when supervisors were relatively older, this effect was less pronounced; it may be that adequately met age-related expectations and a subsequent lack of contrast effect kept employees similarly satisfied and motivated regardless of what kind of feedback they received from a demographically "normal" supervisor. 
Similarly, Hempel's (2008) finding that negative feedback can be attributed to supervisor motivations beyond the employee's actual job performance may be extended here. Hempel (2008) suggested that when Chinese employees did not perceive a positive relationship with their supervisors, they tended to view negative feedback as an indication of the supervisor-subordinate relationship and not as a marker of their own performance. It may be that employees receiving negative feedback from their relatively younger supervisors perceived this to be a message about how the supervisors felt about them, which may have been particularly offensive considering the emphasis on the veneration of older individuals in China (Child \& Markoczky, 1993; Hofstede, 1980). Thus, employees in this situation were less satisfied with and motivated by the feedback they received. Along these lines, relatively older supervisors may have been viewed as more credible by younger employees, such that the feedback reactions of the latter were less affected by feedback valence coming from the former. These ideas should be considered with caution; supervisor-subordinate directional age differences were not found to directly affect LMX scores, and thus there is no indication that individuals with younger supervisors perceived lower quality relationships with those supervisors in this sample. Regardless, future researchers should examine the possibility that supervisor-subordinate directional age 
differences impact the attributions employees make regarding their supervisors' motivations in rating their performance.

Feedback valence was a significant moderator in the relationships between supervisor-subordinate directional age differences and LMX, employee feedback satisfaction, and employee post-feedback motivation, but not informational or interpersonal justice perceptions, or employee perceptions of feedback utility. First, this suggests that justice was perceived by employees as being the same whether the feedback they received was positive or negative. This may have been an artifact of actual events; Chinese values of relationship-building and mutual respect in organizations would suggest that supervisors generally aim to have fair and respectful interactions with their subordinates, regardless of dyadic demographic composition. Alternatively, this may be an artifact of the respect for hierarchy inherent in Chinese culture (Child \& Markoczy, 1993; Fisher \& Yuan, 1998). As previously mentioned, while the reactions measured in the current study were focused toward the feedback event itself, justice perceptions were more focused on ratings of supervisory behaviors during the feedback event. As a result of Chinese values espousing unconditional hierarchical respect, even employees receiving negative feedback may have rated the actions of their supervisor positively. 
Second, these findings suggest that employees found feedback to be similarly useful (and more useful coming from an older supervisor) whether the feedback they received was negative or positive. These findings again point to the importance of respect for hierarchy and the reverence of older individuals in China (Child \& Markoczy, 1993; Hofstede, 1980; 2001), as even employees receiving negative feedback found it to be useful for their improvement when the supervisor delivering this feedback was their older superior.

Further exploratory analyses revealed that valence was not a significant moderator in the relationships between LMX and any of the other study variables of interest (i.e., informational and interpersonal justice perceptions and the three types of employee feedback reactions). This indicates that in the current sample, LMX impacted employee justice perceptions and feedback satisfaction positively regardless of whether the feedback given was positive or negative. LMX did not significantly predict perceptions of feedback utility or post-feedback motivation, again regardless of whether employees received positive or negative feedback. As such, the supervisor-subordinate relationship was pervasive in predicting higher ratings of supervisor behavior and positive affective reactions to the supervisorsubordinate interaction in question (i.e., the feedback event), again indicating that relationship-focused Chinese norms outweighed the more individualistic views that research has shown employees in Western cultures tend to undertake when 
reacting to feedback valence (e.g., Brockner et al., 2001; Ilgen \& Davis, 2000;

Tata et al., 2003).

Finally, valence was not found to significantly moderate the effects of employee informational and interpersonal justice on any of the three types of employee feedback reactions measured. As such, interpersonal justice was a strong predictor of all three feedback reactions whether the feedback itself was positive or negative, while informational justice perceptions did not predict any of the three feedback reactions, again regardless of whether the feedback was positive or negative. Once again these supervisor ratings were pervasive in their effects on feedback reactions. This may indicate that feedback valence, which by itself constitutes an individual performance rating, has a relatively small impact on altering the way feedback reactions are formed in a collectivistic and relationship-focused culture. 


\section{CHAPTER X.}

\section{Implications for Research and Practice}

The findings reviewed in the previous section have important conceptual and practical implications for the field of industrial/organizational psychology, as well as other fields affected by changing workforce demographics throughout the world.

Scientific implications. Conceptually, the current findings suggest that the role of supervisor age relative to employee age in forming work-related attitudes is not as cut-and-dried as previously thought. The significant moderating effects of feedback valence, for instance, demonstrate that different components of a feedback event impact employee reactions differently depending on supervisor relative age, rather than non-normative demographic situations uniformly negatively impacting employee impressions of these events.

Further, the results show that while overall reactions to feedback episodes may be impacted negatively by non-normative demographic supervisorsubordinate dyads, employee interactional justice and LMX perceptions are less affected. Again, this may be a result of cultural norms and values; the current study demonstrates and reiterates the importance of considering culture in organizational research and how it plays a role in workplace relationships. It is possible that strong cultural values are given more weight than demographic or 
relational variables in employee perceptions of supervisor interactions. While future research is needed to determine more explicitly whether this is the case, this possibility may shift our thinking when we conduct research in non-Western cultures.

I interpreted the largely non-significant results of the current study through the lens of Chinese culture, suggesting that cultural values played a role in how employee perceived their supervisors and the feedback administered by those supervisors. However, it is worth noting that even the significant results of the study generally had relatively small effect sizes (the variance of directional age differences accounted for relatively little of the employee feedback reactions), indicating that the relationships I examined were not necessarily relevant to the sampled employees. It is possible that these effects represent Chinese organizational functioning in general, but it is also possible that the results are specific to the characteristics of the current sample.

The study sample came from one organization in one cultural context. Although the organization employs both line workers and engineers, these job types were not distinguished in the current study. Further, this organization represented one industry; there are countless other industries and organization types in which these concepts may differ. For instance, there are certain industries, such as the video game design industry, that reveres younger 
employees and supervisors as sources of innovation (e.g., Wesley \& Barczak, 2010). It is likely that in these types of organizations the study relationships will look completely different. Further, while the current study did not account for job type, it is possible that different jobs will yield different employee reactions to having a younger supervisor. One previously mentioned study found that employees actually had better reactions to their younger supervisors (Vecchio, 1993). This study was conducted with high school faculty, a job type in which career progression typically does not occur linearly with age. Other job types with similar career progression standards may yield similar results.

The current study calls explicitly for more research in China and other cultures to enhance our understanding of how non-normative demographic pairings impact employee attitudes and behaviors. However, researchers also need to assess these relationships in other industries and organizations. This study provided a jumping-off point for this type of research, but much more needs to be done in order for organizational researchers and practitioners to get a complete picture of relatively younger supervisors and their impact on the global workforce.

In the study results, the variable assessing dyad tenure (how long the employee and supervisor had been working together) was largely not significant in impacting the study variables. However, certain aspects of this variable may 
merit further examination. Employees were asked specifically how long they had been working with their current supervisors, but this did not account for how long the supervisor had been their supervisor. Many of the supervisors in question were line managers who had been promoted to their current position over time. Thus, it is possible that employees had worked side-by-wide with their supervisors prior to this promotion.

While the current study did not explicitly account for this, these types of situations may be especially interesting to examine in the context of perceived violations of normative career progression, particularly when the promoted supervisor is younger than the subordinate employee. Some variables that may impact employee attitudes to this situation beyond supervisor age may also include employee perceptions of supervisor expertise, job performance, and education. Promoted supervisors who are perceived as lacking education or jobrelated knowledge may garner especially negative reactions from subordinate employees. Supervisor chronological age may be correlated with some of those variables (for instance, perceived lack of experience), but it certainly may not tell the whole story. Researchers examining the effects of implicit career timetable violations can gain insight from delving into these particular situations and examining the variables within this context that impact employee attitudes. 
The current research has implications for the study area of relational demography in general. Although this study found (limited) support for the idea of perceived violations of implicit career timetables, no support was found for relational demography in particular. Put another way, directional demographic differences were found to be impactful to some extent; general demographic differences were not. The control variable assessing gender congruence (whether the supervisor and subordinate within the dyad were the same gender) had largely non-significant effects on the study variables, indicating that employee perceptions and reactions to performance feedback were not dependent on whether the feedback was administered by someone of the same gender. While this study did not explicitly identify whether general or directional age differences had a larger impact on the study variables, analyses examining absolute age differences (the degree of age difference irrespective of the direction of that difference) showed that general age differences did not predict any of the employee feedback perceptions or reactions.

As such, the current study falls in line with other research that has revealed inconsistent effects of relational demography (e.g., Tsui et al., 1992; Wesselowski \& Mossholder, 1997). Future researchers working within the lens of relational demography should note that directional differences may actually be more impactful on work outcomes. Implicit career timetables provide one theoretical 
framework for examining directional age differences; others may be more appropriate for examining other demographic differences that violate social and cultural norms (e.g., Geddes \& Konrad, 2003).

The current results were interpreted through the lens of Hofstede's cultural dimensions (e.g., collectivism, power distance) (1980). Much of the multi-cultural organizational literature has also used this lens, with Hofstede's work on culture touted as "the most widely cited in existence" (Jones, 2007). However, some critics have argued that Hofstede's cultural dimensions are oversimplified and incomplete.

Problems proposed with Hofstede's framework include its outdated nature (researchers have noted that political changes within countries can cause rapid shifts in organizational cultures and practices, although Hofstede has since argued against this idea) (Hofstede, 1998; Jones, 2007; Nasif, Al-Daeaj, Ebrahimi, \& Thibodeaux, 1991; Newman, 1996). Critics have also argued that the limited dimensions Hofstede (1980) proposed are too broad and vague (Jones, 2007). Further, it has been argued that ascribing a vague label to an entire nation whose regional customs likely vary greatly is a drastically incomplete picture of culture (Dorfman \& Howell 1988; Nasif et al., 1991; Smith, 1998).

It is beyond the scope of the current research to determine whether Hofstede's cultural dimensions continue to apply to the various regions in China; rather, 
these dimensions were used as a guide by which study results were interpreted. However, future researchers conducting cross-cultural studies may note that Hofstede's dimensions are only one (possibly flawed) lens through which to view those cultures.

Lastly, the current study incorporated both justice and LMX employee perceptions, where feedback research has historically examined one or the other (e.g., Erdogan, 2002; Greenberg, 1986). The study analyses were conducted in stages, so that models incorporating multiple feedback-relevant constructs could be compared with models not incorporating some of those constructs. Differences were revealed based on whether analyses explored only the effects of directional age differences, the simultaneous effects of justice perceptions and directional age differences, or the simultaneous effects of age differences, justice, and LMX. Each model revealed new information that painted a more complete picture of employee feedback reactions. This suggests that future researchers should simultaneously examine multiple elements and relevant constructs when exploring the holistic nature of supervisor-subordinate workplace interactions.

Practical implications. On the practical side, these results indicate that in this sample, the effects of having a younger supervisor (i.e., being in a demographically non-normative dyad) may not be as detrimental as previously thought. While having a younger supervisor did impact employee feedback 
satisfaction and utility perceptions negatively, the negative impact on postfeedback employee motivation disappeared once justice perceptions and LMX were considered as part of the model. This indicates that in the current sample, employees were not as satisfied with feedback coming from their younger supervisors, but they remained motivated to work, possibly due to their collectivistic and organization-focused values (Child \& Markoczy, 1993; Fisher \& Yuan, 1998; Hofstede, 1980; 2001). Further, having a younger supervisor did not negatively impact employee interactional justice perceptions or LMX ratings, two determinants of a wealth of important individual and organizational outcomes (Colquitt, 2001; Colquitt et al., 2001; Erdogan, 2002; Greenberg, 1986; 1990; Hui et al., 2008; Schyns et al., 2005; Wayne et al., 1997; Wayne \& Green, 1993). These findings demonstrate that, particularly in cultures where collectivism and personal relationships are revered in the workplace, having a younger supervisor may not present as many deficits in employee attitudes and behaviors as hypothesized (although it is difficult to say from these results whether the findings will generalize to other types of organizations within those cultures). Future researchers may examine other aspects of employee performance not in the context of performance feedback to determine if this is indeed the case.

Conversely, the findings also suggest practical implications for what not to do when relatively younger supervisors negatively impact employee attitudes. LMX 
was not found to moderate the significant, negative relationships between supervisor-subordinate age differences and employee feedback satisfaction and perceptions of feedback utility. As such, it seems that in the current sample, developing a better relationship with one's younger supervisor did not impact the reactions that an employee has to the feedback administered by that supervisor. As previously mentioned, future research warrants the examination of other potential moderators to determine methods of alleviating any negative effects that non-normative demographic pairings may yield. However, the current results suggest that, particularly in a culture similar in values and norms to those practiced in Shenzhen, developing initiatives to improve supervisor-subordinate relationships (which, according to current descriptive results and Chinese customs, are possibly already viewed positively by employees) may not be the most effective method.

One thing that was not considered in the current study was the possibility of a three-way interaction. LMX was not found to significantly moderate the relationships between supervisor-subordinate directional age differences (i.e., having a younger supervisor) and any of the study variables. However, exploratory analyses revealed that valence moderated the relationship between having a younger supervisor and employee ratings of LMX. It is possible that valence, directional age differences, and LMX all vary together to produce 
differences in employee feedback perceptions and reactions. If this is the case, this could yield some interesting insights into the situations and conditions under which employee feedback reactions are formed.

It was beyond the scope of the current study to conduct these types of threeway interactions, as that level of complexity would not have been practically useful (organizational managers being aware that negative feedback, coupled with their age relative to an employee, coupled with variations in employee-perceived LMX produce differences in employee feedback reactions would probably not be inclined to simultaneously consider all of these things prior to each of their employee interactions). However, it may be interesting for future researchers to further examine how various multi-level interactions impact some of the variables examined in this study, allowing us to better understand the affective and cognitive dynamics in a performance feedback event.

Unlike LMX, feedback valence did moderate some of these age differencesfeedback reactions relationships. These significant moderating effects suggest that supervisor-subordinate directional age differences do not always uniformly impact employee perceptions of and reactions to performance feedback. Supervisors administering feedback that varies in terms of valence should consider the impact of the feedback on the employee, paying particular attention to how their relative age may shape this impact. These findings suggest that 
supervisor training may focus on best practices for delivering feedback to both older and relatively younger employees. In light of the current results, supervisors may be encouraged to especially consider the quality of their own relationship with their subordinates when delivering negative feedback to relatively younger employees, and employee satisfaction and motivational impacts when delivering negative feedback to relatively older employees. Such relative age-focused supervisor training may be a valuable tool for organizations as they seek to successfully adapt to the current and impending shifts in workforce demography.

Finally, as previously mentioned, performance appraisal events are important to organizations from a practical standpoint, as they are commonly used to manage employee performance. As such, better and more in-depth knowledge of how to make these events effective and beneficial is essential. This study, as well as other research (e.g., Bianchi \& Ames, 2008; Brett \& Atwater, 2001; Levy \& Williams, 2004; Pearce \& Porter, 1986) has been designed on the premise that garnering more positive employee reactions to performance feedback events will enhance the effectiveness of those events in promoting improved employee performance. This notion has been supported among U.S. employees (e.g., Bianchi \& Ames, 2008; Brett \& Atwater, 2001).

Among Chinese employees, the link between feedback event reactions and subsequent performance is less clear. Virtually no research conducted in China 
has examined this relationship. Although some research has shown that elements such as loyalty to the supervisor, trust in the supervisor, and organizational commitment tend to predict Chinese employee in-role and extra-role work performance (Chen, Tsui, \& Farh, 2002; Dirks \& Ferrin, 2002), these elements have not been examined in the context of a performance feedback event. Research is needed to disentangle whether elements pertaining to feedback events predict subsequent Chinese employee work performance, considering the cultural norms of the nation, in a similar manner as in the United States. If feedback reactions are not as impactful on employee performance in China, it may be that other outcomes, such as performance itself, are more fruitful to examine as we seek to better understand the workplace impacts of non-normative demographic pairings in that nation.

In a similar vein, recent meta-analytic studies have suggested that, compared to the United States, organizational justice may not be as impactful on employee attitudes and behaviors in China. Li and Cropanzano (2009) suggested that justice has a larger bearing on employee attitudes in North America because of their selffocus (as opposed to the other-focused orientation seen among Chinese employees). Shao, Rupp, Skarlicki, and Jones (2013) suggested that perceptions of organizational justice had higher effect sizes on supervisor- and employerrelated outcomes in cultures that were low in power distance and high in 
individualism. While the current study indicated that interpersonal justice perceptions were important in predicting employee feedback reactions, it is possible that practical approaches to improve employee performance in China specifically should focus less on justice characteristics and more on other objective elements that organizations can impact as they seek to drive overall company productivity. Future research will determine what some of these objective elements may be and whether organizational justice can be used at least in conjunction with these elements to impact important organizational outcomes in China and in other similar nations. 


\section{CHAPTER XI.}

\section{Limitations and Directions for Future Research}

The current findings have important contributions to organizational research and practice, as discussed above, but the study has multiple limitations that future researchers may address. First, although the study design employed a time lag, allowing the examination of the formation of employee feedback reactions over time, researchers have suggested that mediation is best tested over at least three time points (Shrout \& Bolger, 2002). The current study required reports of dyad demography, LMX, and ratings of informational and interpersonal justice to be analyzed cross-sectionally. Thus, it cannot be said with confidence that the demographic patterns reported truly caused reduced LMX within a dyad, or that these demographic patterns and LMX caused employee justice perceptions. Rather, the current study provided correlational evidence that these variables were related (or, in some cases, were not related) at one point in time. In the future researchers may address this limitation by examining their temporal occurrence. It would be interesting and informative to examine newly formed supervisorsubordinate dyads and the demographic factors that may potentially impact the formation of LMX over time, especially considering research indicating that demography is particularly important in the initial development of such relationships (Bauer \& Green, 1996; Dienesch \& Liden, 1986). Further, in order 
to establish a true temporal precedence of LMX in relation to employee justice perceptions, these variables will need to be measured at (at least) two different points in time.

The current study utilized self-report measures that asked participants to indicate as accurately and honestly as possible their impressions of their most recent performance appraisal event. Certainly retroactive bias was a potential issue here, particularly when participants were asked to report their feedback reactions 8 weeks after the feedback event had taken place. The surveys were designed to specifically prompt participants to be truthful and honest regarding their perceptions and reactions, but it is possible that they either did not accurately remember the feedback event, or that other factors such as workplace politics contributed to their responding in a certain way (e.g., Harris \& Kacmar, 2005; Podsakoff et al., 2003).

Additionally, because the variables were all self-report, the current study focused entirely on employee perceptions rather than objective elements of the workplace. While employee perceptions are vitally important when examining employee feedback reactions, a better understanding of objective workplace practices that hinder or contribute to positive employee attitudes is important from a practical standpoint. As an example of this limitation, participants in this study were asked to indicate their immediate supervisor's age. While this was intended 
to represent supervisor chronological age (and, therefore, chronological age differences between employees and their supervisors), employee-reported supervisor age may have been colored by employee biases, perceptions, or simply not knowing the accurate information. Thus, it is possible that for some dyads this information was flawed; from a practical perspective, it may be helpful in the future to acquire supervisor and subordinate ages from both sources in order to ensure accuracy. Building on this, future researchers may want to address this limitation by examining not only employee perceptions, but also supervisor perceptions (for instance, of the LMX relationship, as per Schreisheim et al., 1999) and/or outsider ratings of justice during a performance feedback event. "Objective" outcome measures, such as direct indicators of changes in performance over time, could also be useful for examining parts of the proposed conceptual pattern.

The ultimate outcome of the current research was employee feedback reactions reported over a period of time, with affective, cognitive, and motivational elements being examined. As previously mentioned, ultimately it was theorized that these reactions will lead to employee performance, which is what supervisors ideally want to affect with their performance appraisals. The data collected for the proposed study did not include a measure of behavioral reactions (for instance, performance ratings) following the feedback events in 
question. Instead, the logical leap was made that employee affect, cognition, and work motivation following the feedback would likely manifest through the employee's behavior on the job.

Other studies assessing feedback reactions have made a similar leap (e.g., Bianchi \& Ames, 2008; Brett \& Atwater, 2001). However, it is possible that while all of these reactions are occurring within individuals, their performance on the job actually has little to do with them, given research evidence that individuals' attitudes and actions are not always highly correlated (Azjen, 1991; Sutton, 1998), and given that cultural differences may play a role (discussed above). In order to truly understand the impact of supervisor-subordinate relative age on employee behavior, future researchers need to gain access to job performance ratings that assess behavioral feedback reactions as well as those that are psychological.

Interesting insight may also come from examining these relationships with different methods. For instance, a lab study in which older or younger superiors give individuals task-related feedback that is manipulated in terms of its valence and interactional justice content can provide us with new and more experimentally-based information about how employees perceive the age of a supervisor in terms of their impressions and attitudes. Such mixed-source, mixedmethod research would also further alleviate the potential limitation of common 
method bias that occurs with exclusively survey-based research (Podsakoff et al., 2003).

A further area of potential field or laboratory study is the examination of the effects of supervisor bias in conducting performance appraisals. Alongside employee reactions research, one prominent area of study with the aim of developing a greater understanding of feedback effectiveness (or lack thereof) concerns factors that influence supervisor ratings above and beyond true employee performance. Such research has revealed that supervisors may be influenced by the extent that they like and identify with the subordinate being rated (Cascio \& Aguinis, 2005), as well as by workplace politics (Longenecker, Sims, \& Gioia, 1987). The current study focused on employee reactions to feedback, but research has shown that factors such as rater-ratee similarity and familiarity also influence supervisor ratings and the feedback that is delivered in the first place (Duarte et al., 1994; Pulakos, White, Oppler, \& Borman, 1989). Social Identity Theory (Tajfel \& Turner, 1986) fits well with this line of research, as in-group/out-group perceptions (formed by such factors as demography and relational quality) are likely to impact supervisor liking of a subordinate (Brewer \& Kramer, 1985; Tajfel \& Turner, 1986).

Some research has indicated that supervisor-subordinate age differences impact supervisor ratings, with older subordinates tending to get higher objective 
and subjective performance ratings (Liden et al., 1996) and supervisors tending to rate subordinates who are close in age to themselves more highly (Borman, White, \& Dorsey, 1995; Cascio \& Aguinis, 2005). To date, no research has been conducted comprehensively examining the effects of supervisor-subordinate directional age differences and their effect on supervisor ratings and employee perceptions of those ratings. Because these phenomena happen simultaneously in the workplace and together impact a variety of important organizational outcomes such as promotions, pay, and employee attitudes (e.g., Brett \& Atwater, 2001; Cascio \& Aguinis, 2005), research assessing both is necessary.

Another possible limitation of the current study concerns the generalizability of the sample. The employees surveyed were mostly male, Chinese, and working within one large organization in China. The latter is a limitation that needs to be remedied by further replications of this research; the former fits one of the purposes of the study - assessing business practices and employee behavior in China - but this also created some potential limitations to the study's results. Given the strong cultural and organizational differences between the United States and China (Dessler \& Tan, 2006; Farh et al., 1998; Hofstede, 2001; Tsui \& O'Reilly, 1989), the current results may not apply to supervisor-subordinate dyads in American organizations in particular. 
One particular discrepancy between the two nations pertinent to the current study is in the existence and prevalence of Guanxi. Guanxi is a Chinese construct that refers to the two-way ties between individuals, with a direct emphasis on roles and "doing one's part" (Farh et al., 1998). Guanxi has a strong focus on the interpersonal relationship between two people, and, in the context of the supervisor-subordinate dyad, is very similar to LMX (Law, Wong, Wang, \& Wang, 2000). However, research has shown that Guanxi is actually distinct from LMX, and has additional explanatory power in predicting supervisory decisions such as promotion and bonus pay (Law et al., 2000). Research has also shown that Guanxi is particularly important in examining workplace relationships between laterally positioned individuals (for instance, the relationship between two managers), while factors such as relational demography in addition to Guanxi influence attitudes in vertical dyads (Farh et al., 1998). However, given the importance of this construct in workplace relationships in China, it may be a fruitful area for future researchers to consider as they attempt to better understand supervisor-subordinate interactions in this nation.

Despite this and other cultural differences, China is similar to the United States in its changing demographic workforce trends. Moreover, increasing globalization and outsourcing indicate that we need to better understand workers from all different cultures and nations if we are to work with them effectively 
(and, in this case, manage and deliver feedback effectively). However, future researchers can make more concrete cross-cultural comparisons by examining these relationships in organizations in other nations, the United States included, to determine whether the strong cultural effects suggested here hold up in a variety of nations with a variety of traditions and norms. As previously mentioned, this cross-cultural research should happen alongside other research examining these relationships in other industries and job types.

Also previously mentioned, future researchers would greatly benefit the science and practice of organizational psychology by identifying moderators that reduce the negative impact of having a younger supervisor on employee attitudes. While LMX was not found to be a moderator here, climate may certainly have some effect, with more permissible age diversity climate promoting greater employee openness toward demographically unusual relationships at work. Another possibility is that dynamics within an employee's work team will alleviate negative effects of non-normative dyadic demographic patterns. Research has shown that if team commitment and cohesion are present, outcomes in terms of employee performance and functioning tend to be positive (Jackson \& Joshii, 2011). If this is the case irrespective of supervisor attributes, organizations employing younger supervisors may focus on team building as a means of maintaining high employee performance. Future researchers should further 
examine these possibilities when investigating the management of employee performance and attitudes in the face of precipitous organizational and workforce demographic changes.

Along these lines, another aspect to consider in supervisor-subordinate interactions is employee age, directly. Employee age has been associated with better contextual and safety performance ( $\mathrm{Ng} \&$ Feldman, 2008) and better work attitudes (Ng \& Feldman, 2010). In the current study, including employee age in the analyses completely eliminated the effect of supervisor-subordinate directional age differences on each of the employee feedback reactions. (Supervisor age, on the other hand, did not have the same nullifying effects.) This may have been a function of the current sample, in which employee age was extremely highly correlated with dyadic age differences $(r=0.79, p<0.01)$, such that being an older employee correlated very highly with having a younger supervisor. The oldest employees in the sample were exclusively paired with younger supervisors, inflating this correlation. Because of these nuances, employee age by itself was excluded from current analyses; future researchers may examine its role in the hypothesized relationships in samples that are more heterogeneous in terms of age and dyadic age differences composition.

Finally, the current study examined performance appraisal events in particular because of their continued importance in employee performance management 
(e.g., Cascio \& Aguinis, 2005). However, this is just one aspect of supervisorsubordinate functioning. Future researchers may examine some of these other types of interactions and how dyad demography impacts these. Further, the exploration of supervisor-subordinate dyads may be extended into less formal arrangements. For instance, it would be interesting to study mentoring relationships in China (and beyond), exploring the impact of relative age in these relationships. Given Chinese norms and values esteeming older individuals (e.g., Dessler \& Tan, 2006; Hofstede, 1980; 2001), the pairing of a younger mentor with an older individual could yield some interesting results. 


\section{CHAPTER XII.}

\section{Conclusion}

What we commonly think of as "work" has changed considerably over the last several decades. Advances in technology and, subsequently, increased globalization have created a work landscape in which organizations and their employees cross national boundaries to accomplish their strategic goals (Dessler \& Tan, 2006; Liebold \& Voelpel, 2006). In conjunction with these changes, shifting demographic trends in developed and developing nations all over the globe are creating a workforce that looks different than it has in the past. One result of these trends is the increasing frequency with which demographically "non-normative" supervisor-subordinate pairings are occurring in the workplaces of nations worldwide (Hirsch, 1990; Mor Barak, 2011).

On one hand, more opportunities are available now for relatively younger employees than there have ever been before. Where traditionally chronological age dictated one's career progression, younger employees, as a result of enhanced education and technical knowledge, can now more easily reach organizational levels where they are managing others (Mor Barak, 2011). On the other hand, these levels often carry with them the weight of managing relatively older employees. Research has indicated that these situations have the potential to unfold negatively, with older employees experiencing more negative attitudes and 
behavioral reactions as a result of being managed by a younger individual (Collins et al., 2009; Perry et al., 1999; Shore et al., 2003). One theoretical explanation for this effect is the idea of implicit career timetables, which suggests that people implicitly form ideas about "normal" career progression, and these ideas often center on chronological age (Lawrence, 1984; 1990; Sofer, 1970).

As demographic trends continue to shift toward increases in non-normative supervisor-subordinate pairings, eventually the "non-normative" nature of these pairings will disappear, and this will become part of the norm. For future generations of workers who did not experience traditional workplace values revering age-related career progression, the impacts of these pairings may not be as detrimental. As such, it would be interesting to examine changes in age diversity climate over time, as older generations leave the workplace and new generations come in. It is likely that as more and more young adults step into management roles, employee perceptions of these adults will become universally more positive. However, this does not indicate that these non-normative demographic pairings are not currently a concern - the actions that organizational researchers and practitioners take in light of the changing demographic trends today will likely impact the way these relationships are perceived and understood in the future. 
The current study examined one aspect of supervisor-subordinate functioning, a formal performance feedback event, and how supervisor-subordinate directional age differences impacted employee reactions to this aspect. The results showed that while having a relatively younger supervisor did impact some employee reactions to the performance feedback event negatively, the mediating and moderating mechanisms that were explored had no bearing on this relationship. Relatively older employees did not appear to be dissatisfied with the feedback event because they perceived reduced justice coming from their younger supervisors, or because they perceived lower-quality relationships with those younger supervisors. Further, when relatively older employees perceived higherquality relationships with their younger supervisors, this did not help to alleviate the negative impact on their feedback event reactions. Other potential mediators and moderators of these negative relationships were discussed here; future researchers are left to decide which of these will be the most fruitful to examine.

The current study examined these direct, mediating and moderating relationships in a nation that is experiencing a shift in its workforce demographic landscape, similar to the changes that many other nations around the world are facing today. As such, cultural norms and values were given considerable weight when interpreting the research findings. These results highlight the importance of considering cultural traditions and customs, which likely impact organizational 
functioning from the way leadership is conducted to the attitudes and reactions employees have, in organizational research. The results also highlight the importance of conducting cross-cultural research comparing different nations, industries, and organizations in terms of their organizational functions and behaviors if we are to truly understand organizational behavior on a global scale.

To summarize, the current study revealed that, within one particular organization in China, younger supervisors tended to garner poorer feedback reactions from employees, but not poorer employee perceptions of feedback event justice or general LMX. Further, the negative impact of having a younger supervisor on employee feedback reactions was not mitigated by LMX, indicating that having a better relationship with one's younger supervisor did not affect how employees reacted to feedback coming from that supervisor. These results provided some support for the idea that directional age differences, and not age differences in general, impact employee attitudes regarding performance appraisal events. Further, this study allowed the examination of previously-established relationships and theories in the cultural context of China.

This study is only a small part of a larger initiative to better understand and predict the impact of non-normative demographic pairings on feedback-related outcomes, and as such should be used as a base for other similar research. Given the changing demographic trends in today's workforce and the importance of 
performance appraisal events in general, such understanding is necessary for organizations who want to remain competitive in a changing world of work. Researchers need to examine these relationships in organizations around the world if we are to inform organizational practitioners in a relevant, current way that allows them to manage their workforces today and plan for the changes of tomorrow. 


\section{References}

Ayree, S. \& Chen, Z. X. (2006). Leader-member exchange in a Chinese context: Antecedents, the mediating role of psychological empowerment and outcomes. Journal of Business Research, 59, 793-801.

Azjen, I. (1991). The theory of planned behavior. Organizational Behavior and Human Decision Processes, 50, 179-211.

Bailey, J. R., Chen, C. C., \& Dou, S-G. (1997). Conceptions of self and performance-related feedback in the U.S., Japan and China. Journal of International Business Studies, 28, 605-625.

Bakker, A. B., Demerouti, E., \& Euwama, M. C. (2005). Job resources buffer the impact of job demands on burnout. Journal of Occupational Health Psychology, 10, 170-180.

Balcazar, F., Hopkins, B. L., \& Suarez, Y. (1986). A critical, objective review of performance feedback. Journal of Organizational Behavior Management, 7, $65-83$.

Barling, J., Christie, A., \& Hoption, C. (2011). Leadership. In. S. Zedeck (Ed.), APA Handbook of Industrial and Organizational Psychology (Vol. 1; pp. 183240). Washington, DC, US: American Psychological Association.

Baron, R. A. (1993). Criticism (informal negative feedback) as a source of perceived unfairness in organizations: Effects, mechanisms, and 
countermeasures. In R. Cropanzano (Ed.), Justice in the Workplace:

Approaching fairness in human resource management: 155-170. Hillsdale, NJ: Erlbaum.

Bauer, T. N. \& Green, S. G. (1996). Development of leader-member exchange: A longitudinal test. Academy of Management, 39, 1538-1567.

Bianchi, E. \& Ames, D. R. (2008, August). What you say and how you say it: The impact of feedback valence, content, and delivery. Paper presented at the 2008 Academy of Management Annual Meeting, Anaheim, CA.

Borman, W. C., White, L. A., \& Dorsey, D. W. (1995). Effects of ratee task performance and interpersonal factors on supervisor and peer performance ratings. Journal of Applied Psychology, 80, 168-177.

Brett, J. F. \& Atwater, L. E. (2001). 360 degree feedback: Accuracy, reactions, and perceptions of usefulness. Journal of Applied Psychology, 86, 930-942.

Brewer, M. B. (1979). In-group bias in the minimal intergroup situation: A cognitive-motivational analysis. Psychological Bulletin, 86, 307-324.

Brewer, M. B. \& Kramer, R. M. (1985). The psychology of intergroup attitudes and behavior. Annual Review of Psychology, 36, 219-243.

Brockner, J., Ackerman, G., Greenberg, J., \& Gelfand, M. J. (2001). Culture and procedural justice: Influence of power distance on reactions to voice. Journal of Experimental Social Psychology, 37, 300-315. 
Burlacu, G., Wang., M., James, K., Truxillo, D. M., \& Yao, X. (2012). Age differences in feedback reactions: The roles of feedback valence, content quality, and feedback delivery. Unpublished manuscript, Portland State University.

Campbell, J. P., Dunnette, M. D., Lawler, E. E., III., \& Weick, K. E., Jr. (1970). Managerial behavior, performance, and effectiveness. New York: McGrawHill.

Cascio, W. F. \& Aguinis, H. (2005). Applied Psychology in Human Resource Management ( $6^{\text {th }}$ Ed.). Upper Saddle River, NJ: Prentice Hall.

Chen, Z. X., Tsui, A. S., \& Farh, J-L. (2002). Loyalty to supervisor vs. organizational commitment: Relationships to employee performance in China. Journal of Occupational and Organizational Psychology, 75, 339-356.

Cheng, B., Chou, L., \& Wu, T. (2004). Paternalistic leadership and subordinate responses: Establishing a leadership model in Chinese organizations. Asian Journal of Social Psychology, 7, 89-117.

Child, J. \& Markoczy, L. (1993). Host country managerial behavior and learning in Chinese and Hungarian joint ventures. Journal of Management Studies, 30, 611-631. 
Chow, I. H. (1995). An opinion survey of performance appraisal practices in Hong Kong and the People's Republic of China. Asia Pacific Journal of Human Resources, 32, 67-79.

Cohen, J., Cohen, P., West, S. G., \& Aiken, L. S. (2003). Applied multiple regression/correlation analysis for the behavioral sciences. Mahwah, NJ: Lawrence Earlbaum.

Collins, M. H., Hair, J. F., \& Rocco, T. S. (2009). The older-worker-youngersupervisor dyad: A test of the Reverse Pygmalion effect. Human Resource Development Quarterly, 20, 21-41.

Colquitt, J. A. (2001). On the dimensionality of organizational justice: A construct validation of a measure. Journal of Applied Psychology, 86, 386400.

Colquitt, J. A., Conlon, D. E., Wesson, M. J., Porter, C. O. L. H., \& Ng, K. Y. (2001). Justice at the millennium: A meta-analytic review of 25 years of organizational justice research. Journal of Applied Psychology, 86, 425-445.

Cropanzano, R., Prehar, C. A., \& Chen, P. Y. (2002). Using social exchange theory to distinguish procedural from interactional justice. Group Organization Management, 27, 324-351.

Dansereau, F., Jr., Graen, G., \& Haga, W. J. (1975). A vertical dyad linkage approach to leadership within formal organizations: A longitudinal 
DIRECTIONAL AGE DIFFERENCES IN CHINA

investigation of the role-making process. Organizational Behavior and Human Performance, 13, 46-78.

Dessler, G. \& C. H. Tan (Eds.). Human Resource Management: An Asian Perspective (Second Edition). 2006.

Dienesch, R. M. \& Liden, R. C. (1986). Leader-member exchange model of leadership: A critique and further development. Academy of Management Review, 11, 618-634.

Dirks, K. T. \& Ferrin, D. L. (2002). Trust in leadership: Meta-analytic findings and implications for research and practice. Journal of Applied Psychology, 87, 611-628.

Dobbins, G. H., Cardy, R. L., \& Platz-Vieno, S. J. (1990). A contingency approach to appraisal satisfaction: An initial investigation of the joint effects of organizational variables and appraisal characteristics. Journal of Management, 16, 619-632.

Dorfman, P. W. \& Howell, J. P. (1988). Dimensions of national culture and effective leadership patterns: Hofstede revisited. Advances in International Comparative Management, 3, 127-150.

Duarte, N. T., Goodson, J. R., \& Klich, N. R. (1994). Effects of dyadic quality and duration on performance appraisal. Academy of Management Journal, 37, 499-521. 
Early, P. C. (1993). East meets West meets Mideast: Further explorations of collectivistic and individualistic work groups. Academy of Management Journal, 36, 319-348.

Elicker, J. D., Levy, P. E., \& Hall, R. J. (2006). The role of leader-member exchange in the performance appraisal process. Journal of Management, 32, $531-551$.

Emerson, R. M. (1976). Social exchange theory. Annual Review of Sociology, 2, 335-362.

Epitropaki, O. \& Martin, R. (1999). The impact of relational demography on the quality of leader-member exchanges and employee's work attitudes and wellbeing. Journal of Occupational and Organizational Psychology, 72, 237-240.

Epitropaki, O. \& Martin, R. (2005). From ideal to real: A longitudinal study of the role of implicit leadership theories on leader-member exchanges and employee outcomes. Journal of Applied Psychology, 90, 659-676.

Erdogan, B. (2002). Antecedents and consequences of justice perceptions in performance appraisals. Human Resource Management Review, 12, 555-578.

Farh, J-L., Tsui, A. S., Xin, K., \& Cheng, B-S. (1998). The influence of relational demography and quanxi: The Chinese case. Organization Science, 9, 471-488. 
Farr, J. L. (1993). Informational performance feedback: Seeking and giving. In H. Schuler, J. L. Farr, \& J. M. Smith (Eds.), Personnel selection and assessment (pp. 163-180). Washington DC: Business \& Economics.

Ferris, G. R. (1985). Role of leadership in the employee withdrawal process: A constructive replication. Journal of Applied Psychology, 70, 777-781.

Festinger, L. (1954). A theory of social comparison processes. Human Relations, 7, 117-140.

Fisher, C. D. \& Yuan, A. X. Y. (1998). What motivates employees? A comparison of US and Chinese responses. International Journal of Human Resource Management, 9, 516-528.

Ford, J. K. \& Noe, R. A. (1987). Self-assessed training needs: The effects of attitudes toward training, managerial level, and function. Personnel Psychology, 40, 39-53.

Geddes, D. \& Konrad, A. M. (2003). Demographic differences and reactions to performance feedback. Human Relations, 56, 1485-1513.

Graen, G., \& Cashman, J. F. (1975). A role-making model of leadership in formal organizations: A developmental approach. In J. G. Hunt \& L. L. Larson (Eds.), Leadership frontiers (pp. 143-165). Kent, OH: Kent State University Press. 
Graen, G. B., Liden, R. C., \& Hoel, W. (1982). Role of leadership in the employee withdrawal process. Journal of Applied Psychology, 67, 868-872.

Graen, G., Novak, M. A., \& Sommerkamp, P. (1982). The effects of leadermember exchange and job design on productivity and satisfaction: Testing a dual attachment model. Organizational Behavior and Human Performance Decision, 30, 109-131.

Graen, G. B. \& Uhl-Bien, M. (1995). Relationship-based approach to leadership: Development of leader-member exchange (LMX) theory of leadership over 25 years: Applying a multi-level multi-domain perspective. The Leadership Quarterly, 6, 219-247.

Green, S. G., Anderson, S. E., \& Shivers, S. L. (1996). Demographic and organizational influences on leader-member exchange and related work attitudes. Organizational Behavior and Human Decision Processes, 66, 203214.

Greenberg, J. (1986). Determinants of perceived fairness of performance evaluations. Journal of Applied Psychology, 71, 340-342.

Greenberg, J. (1990). Organizational justice: Yesterday, today, and tomorrow. Journal of Management, 16, 399-432. 
Greller, M. M. \& Nee, D. M. (1989). From baby boom to baby bust: How business can meet the demographic challenge. Reading, MA: AddisonWesley.

Harris, K. J. \& Kacmar, K. M. (2005). Easing the strain: The buffer role of supervisors in the perceptions of politics-strain relationship. Journal of Occupational and Organizational Psychology, 78, 337-354.

Harrison, D. A., Price, K. H., \& Bell, M. P. (1998). Beyond relational demography: Time and the effects of surface- and deep-level diversity on work group cohesion. Academy of Management Journal, 41, 96-107.

Hempel, P. S. (2008). Chinese reactions to performance feedback: Non-task attributions of feedback intentions. Asia Pacific Journal of Human Resources, 46, 196-219.

Herr, P. M., Sherman, S. J., \& Fazio, R. H. (1983). On the consequences of priming: Assimilation and contrast effects. Journal of Experimental Social Psychology, 19, 323-340.

Hicks-Clarke, D. \& Iles, P. (2000). Climate for diversity and its effects on career and organizational attitudes and perceptions. Personnel Review, 29, 324-345.

Hirsch, J. S. (1990, February). Older workers chafe under younger managers. Wall Street Journal, B1, B6. 
Hofstede, G. (1980). Culture's consequences: International differences in workrelated values. Beverly Hills, CA: Sage.

Hofstede, G. (1998). Attitudes, values, and organizational culture: Disentangling the concepts. Organization Studies, 19, 477.

Hofstede, G. (2001). Culture's consequences: Comparing values, behaviors, institutions, and organizations across nations $\left(2^{\text {nd }}\right.$ ed). Thousand Oaks, CA: Sage Publications.

Hovland, C. I., Harvey, O. J., \& Sherif, M. (1957). Assimilation and contrast effects to communication and attitude change. Journal of Abnormal and Social Psychology, 55, 244-252.

Hui, C., Law, K. S., Chen, N. Y. F., \& Tjosvold, D. (2008). The role of cooperation and competition on leader-member exchange and extra-role performance in China. Asia Pacific Journal of Human Resources, 46, 133 152.

Hui, C., Lee, C., \& Rousseau, D. M. (2004). Psychological contract and organizational citizenship behavior in China: Investigating generalizability and instrumentality. Journal of Applied Psychology, 89, 311-321.

Hui, W., Xiongying, N., \& Law, K. S.(2004). Multi-dimensional leader-member exchange (LMX) and its impact on task performance and contextual performance of employees. Acta Psychologica Sinica, 2. 
Ilgen, D. \& Davis, C. (2000). Breaking bad news: Reactions to negative performance feedback. Journal of Applied Psychology, 49, 550-565.

Ilgen, D.R., Fisher, D.D., \& Taylor, M.S. (1979). Consequences of individual feedback on behavior in organizations. Journal of Applied Psychology, 64, 349-371.

Jackson, S. E. \& Joshii, A. (2011). Work team diversity. In S. Zedeck (Ed.), APA handbook of industrial and organizational psychology, Vol. 1: Building and developing the organization. Washington, DC, US: American Psychological Association.

James, L. R. \& Jones, A. P. (1974). Organizational climate: A review of theory and research. Psychological Bulletin, 81, 1096-1112.

Jones, M. L. (2007). Hofstede-Culturally questionable? Paper presented at the Oxford Business and Economics Conference, June 2007.

Judge, T. A., Thoreson, C. J., Bono, J. E., \& Patton, G. K. (2001). The job satisfaction-job performance relationship: A qualitative and quantitative review. Psychological Bulletin, 127, 376-407.

Kelloway, E. K. \& Barling, J. (2010). Leadership development as an intervention in occupational health psychology. Work \& Stress, 24, 260-279. 
Kirchmeyer, C. (1995). Demographic similarity to the work group: A longitudinal study of managers at the early career stage. Journal of Organizational Behavior, 16, 67-83.

Kluger, A. N. \& DeNisi, A. (1996). The effects of feedback interventions on performance: A historical review, a meta-analysis, and a preliminary feedback intervention theory. Psychological Bulletin, 119, 254-284.

Lam, T. (2003). Leader-member exchange and team-member exchange: The roles of moderators in new employees' socialization. Journal of Hospitality and Tourism Research, 27, 48-68.

Larson, J. R. (1989). The dynamic interplay between employees' feedback seeking strategies and supervisors' delivery of performance feedback. Academy of Management Review, 14, 408-422.

Law, K. S., Wong, C., Wang, D., \& Wang, L. (2000). Effect of supervisorsubordinate quanxi on supervisory decisions in China: an empirical investigation. The International Journal of Human Resource Management, 11, $751-765$.

Lawrence, B. S. (1984). Age grading: The implicit organizational timetable. Journal of Organizational Behavior, 5, 23-35.

Lawrence, B. S. (1988). New wrinkles in the theory of age: Demography, norms and performance ratings. Academy of Management, 31, 209-227. 
Lawrence, B. S. (1990). At the crossroads: A multiple-level explanation of individual attainment. Organization Science, 1, 65-85.

Leow, K. L. \& Kuong, K. W. (2009). Organizational commitment: The study of organizational justice and leader member exchange (LMX) among auditors in Malaysia. International Journal of Business and Information, 4, 161-198.

Leung, K., Smith, P. B., Wang, Z., \& Sun, H. (1996). Job satisfaction in joint venture hotels in China: An organizational justice analysis. Journal of International Business Studies, 27, 947-962.

Leung, K., Su, S., \& Morris, M. W. (2001). When is criticism not constructive? The role of fairness perceptions and dispositional attributions in employee acceptance of critical supervisory feedback. Human Relations, 54, 1155-1187.

Levy, P. E. \& Williams, J. R. (2004). The social context of performance appraisal: A review and framework for the future. Journal of Management, 30, 881-905.

Li, A. \& Cropanzano, R. (2009). Do East Asians respond more/less strongly to organizational justice than North Americans? A meta-analysis. Journal of Management Studies, 46, 787-805.

Li, M. C. \& Hsu, C. S. (1995). In-group favoritism and development of communal identity: The case of college students in Taiwan. Paper presented at the Biannual Meeting of Chinese Psychologist, Taipei. 
Liao, H. \& Rupp, D. E. (2005). The impact of justice climate and justice orientation on work outcomes: A cross-level multifoci framework. Journal of Applied Psychology, 90, 242-256.

Liden, R. C., \& Maslyn, J. M. (1998). Multidimensionality of leader-member exchange: An empirical assessment through scale development. Journal of Management, 24, 43-72.

Liden, R. C., Stilwell, D., \& Ferris, G. R. (1996). The effects of supervisor and subordinate age on objective performance and subjective performance ratings. Human Relations, 49, 327-347.

Liebold, M. \& Voepel, S. (2006). Managing the aging workforce: Challenges and solutions. Germany: Wiley Publishing.

Ling, W., Chia, R. C., \& Fang, L. (2000). Chinese implicit leadership theory. The Journal of Social Psychology, 140, 729-739.

Longenecker, C. O., Sims, H. P., \& Gioia, D. A. (1987). Behind the mask: The politics of employee appraisal. Academy of Management Executive, 1, 183193.

Lord, R. G., Foti, R. J., \& DeVader, C. L. (1984). A test of leadership categorization theory: Internal structure, information processing, and leadership perceptions. Organizational Behavior and Human Performance, 34, 343-378. 
Lord, R. G., De Vader, C. L., \& Alliger, G. M. (1986). A meta-analysis of the relation between personality traits and leadership perceptions: An application of validity generalization procedures. Journal of Applied Psychology, 71, 402410.

Mabe, P. \& West, S. (1982). Validity of self-evaluation of ability: A review and meta-analysis. Journal of Applied Psychology, 67, 280-296.

Martin, R., Thomas, G., Charles, K., Epitropaki, O., \& McNamara, R. (2005). The role of leader-member exchanges in mediating the relationship between locus of control and work reactions. Journal of Occupational and Organizational Psychology, 78, 141-147.

Masterson, S. S., Lewis, K., Goldman, B. M., \& Taylor, M. S. (2000). Integrating justice and social exchange: The differing effects of fair procedures and treatment on work relationships. Academy of Management Journal, 43, 738748.

Milliken, F. J. \& Martins, L. L. (1996). Searching for common threads:

Understanding the multiple effects of diversity in organizational groups. Academy of Management Review, 21, 402-433.

Mok, K-H. (2002). Policy of decentralization and changing governance of higher education in post-Mao China. Public Administration and Development, 22, 261-273. 
Mor Barak, M. E. (2011). Managing diversity: Toward a globally inclusive workplace (2nd ed.) Thousand Oaks, CA: Sage Publishing.

Mowday, R. T., Porter, L. W., \& Dubin, R. (1974). Unit performance, situational factors, and employee attitudes in spatially separated work units. Organizational Behavior and Human Performance, 12, 231-248.

Nasif, E. G., Al-Daeaj, H., Ebrahimi, B., \& Thibodeaux, S. (1991). Methodological problems in cross-cultural research: An update. Management International Review, 31, 79-95.

Naumann, S. E. \& Bennett, N. (2000). A case for procedural justice climate: Development and test of a multilevel model. Academy of Management, 43, 881-889.

Newman, K. L. (1996). Culture and congruence: The fit between management practices and national culture. Journal of International Business Studies, 27, 753.

Ng., T. W. H. \& Feldman, D. C. (2008). The relationship of age to ten dimensions of job performance. Journal of Applied Psychology, 93, 392-423.

Ng, T. W. H. \& Feldman, D. C. (2010). The relationships of age with job attitudes: A meta-analysis. Personnel Psychology, 63, 677-718.

Noe, R. A., Noe, A. W., \& Bachhuber, J. A. (1990). An investigation of the correlates of career motivation. Journal of Vocational Behavior, 37, 340-356. 
Nolan, P. (2002). China and the global business revolution. Cambridge Journal of Economy, 26, 119-137.

Offermann, L. R., Kennedy, J. K., \& Wirtz, P. W. (1994). Implicit leadership theories: Content, structure, and generalizability. Leadership Quarterly, 5, 4358.

Ostroff, C. (1992). The relationship between satisfaction, attitudes, and performance: An organizational level analysis. Journal of Applied Psychology, 77, 963-974.

Pearce, J. L. \& Porter, L. W. (1986). Employee responses to formal performance appraisal feedback. Journal of Applied Psychology, 71, 211-218.

Pearsall, M. J., Ellis, A. P. J., \& Evans, J. M. (2009). Unlocking the effects of gender faultlines on team creativity: Is activation the key? Journal of Applied Psychology, 93, 225-234.

Pelled, L. H. (1996). Relational demography and perceptions of group conflict and performance: A field investigation. International Journal of Conflict Management, 7, 230-246.

Pelled, L. H. \& Xin, K. R. (2000). Relational demography and relational quality in two cultures. Organization Studies, 21, 1077-1094. 
Perry, E. L., Kulik, C. T., \& Zhou, J. (1999). A closer look at the effects of subordinate-supervisor age differences. Journal of Organizational Behavior, 20, 341-357.

Podsakoff, P. M. \& Farh, J-L. (1989). Effects of feedback sign and credibility on goal setting and task performance. Organizational Behavior and Human Decision Processes, 44, 45-67.

Podsakoff, P. M., MacKenzie, S. B., Lee, J. Y., \& Podsakoff, N. P. (2003). Common method bias in behavioral research: A critical review of the literature and recommended remedies. Journal of Applied Psychology, 88, 879-903.

Pulakos, E. D., White, L. A., Oppler, S. H., \& Borman,W. C. (1989). Examination of race and sex effects on performance ratings. Journal of Applied Psychology, 74, 770-780.

Riordan, C. M. (2000). Relational demography within groups: Past developments, contradictions, and new directions. Research in Personnel and Human Resources Management, 19, 131-173.

Riordan, C. M. \& Shore, L. M. (1997). Demographic diversity and employee attitudes: An empirical examination of relational demography within work units. Journal of Applied Psychology, 82, 324-358. 
Roberson, Q. M. \& Stewart, M. M. (2006). Understanding the motivational effects of procedural and informational justice in feedback processes. British Journal of Psychology, 97, 281-298.

Scandura, T. A. (1999). Rethinking leader-member exchange: An organizational justice perspective. Leadership Quarterly, 10, 25-41.

Scandura, T. A., \& Graen, G. B. (1984). Moderating effects of initial leadermember exchange status on the effects of a leadership intervention. Journal of Applied Psychology, 69, 428-436.

Scandura, T. A., Graen, G. B., \& Novak, M. A. (1986). When managers decide not to decide autocratically: An investigation of leader-member exchange and decision influence. Journal of Applied Psychology, 71, 579-584.

Schaffer, B. S. \& Riordan, C. M. (2003). A review of cross-cultural methodologies for organizational research: A best-practices approach. Organizational Research Methods, 6, 169-215.

Schaubroeck, J. \& Lam, S. S. K. (2002). How similarity to peers and supervisor influences organizational advancement in different cultures. Academy of Management Journal, 45, 1120-1136.

Schreisheim, C. A., Castro, S. L., \& Cogliser, C. C. (1999). Leader-member exchange (LMX) research: A comprehensive review of theory, measurement, and data-analytic practices. Leadership Quarterly, 10, 63-113. 
Schyns, B., Paul. T., Mohr, G., \& Blank, H. (2005). Comparing antecedents and consequences of leader-member exchange in a German working context to findings in the US. European Journal of Work and Organizational Psychology, 14, 1-22.

Shao, R., Rupp, D. E., Skarlicki, D. P., \& Jones, K. S. (2013). Employee justice across cultures: A meta-analytic review. Journal of Management, 39, $263-$ 300.

Sherif, M. \& Hovland, C. I. (1961). Social judgment: Assimilation and contrast effects in communication and attitude change. New Haven: Yale University Press.

Sherman, S. J., Ahlm, K., Berman, L., \& Lynn, S. (1987). Contrast effects and their relationship to subsequent behavior. Journal of Experimental Social Psychology, 14, 340-350.

Shore, L. M., Cleveland, J. N., \& Goldberg, C. B. (2003). Work attitudes and decisions as a function of manager age and employee age. Journal of Applied Psychology, 88, 529-537.

Shrauger, J. S. (1975). Responses to evaluation as a function of initial selfperceptions. Psychological Bulletin, 82, 581-596. 
Shrout, P. E. \& Bolger, N. (2002). Mediation in experimental and nonexperimental studies: New procedures and recommendations. Psychological Methods, 7, 422-445.

Smith, M. (1998). Culture and organizational change. Management Accounting, $76,60-78$.

Sofer, C. (1970). Men in mid-career: A study of British managers and technical specialists. Great Britain: Cambridge University.

Streib, G. F. (1987). Old age in sociocultural context: China and the United States. Journal of Aging Studies, 1, 95-112.

Sutton, R. S. (1998). Reinforcement learning: An introduction. Boston, MA: Cambridge University Press.

Tajfel, H. \& Turner, J. C. (1986). The social identity theory of intergroup behavior. In S. Worchel \& W. G. Austin (Eds.), Psychology of intergroup relationships (pp. 7-24). Chicago: Nelson-Hall.

Tata, J., Fu, P. P., \& Wu, R. (2003). An examination of procedural justice principles in China and the US. Asia Pacific Journal of Management, 20, 205216.

Taylor, M. S., Fisher, C. D., \& Ilgen, D. R. (1984). Individuals' reactions to performance feedback in organizations: A control theory perspective. In K. M. 
Rowland \& G. R. Ferris (Eds.), Research in personnel and human resources management (pp. 81-124). Greenwich, CT: JAI Press.

Taylor, M. S., Tracy, K. B., Renard, M. K., Harrison, J. K., \& Carroll, S. J. (1995). Due process in performance appraisal: A quasi-experiment in procedural justice. Administrative Science Quarterly, 40, 495-523.

Tekleab, A. G., Takeuchi, R., \& Taylor, M. S. (2005). Extending the chain of relationships among organizational justice, social exchange, and employee reactions: The role of contract violations. Academy of Management Journal, 48, 146-157.

Thorndike, E.L. (1927). The law of effect. American Journal of Psychology, 39, 212-222.

Timmerman, T. A. (2000). Racial diversity, age diversity, interdependence, and team performance. Small Group Research, 31, 592-606.

Tsui, A. S., Egan, T. D., \& O’Reilly, C. A. (1992). Being different: Relational demography and organizational attachment. Administrative Science Quarterly, 37, 549-579.

Tsui, A. S., Nifadkar, S. S., \& Ou, A. Y. (2007). Cross-national, cross-cultural organizational behavior research: Advances, gaps, and recommendations. Journal of Management, 33, 426-478. 
Tsui, A. S. \& O’Reilly, C. A. (1989). Beyond simple demographic effects: The importance of relational demography in superior-subordinate dyads. Academy of Management Journal, 32, 402-423.

Tsui, A. S., Porter, L. W., \& Egan, T. D. (2002). When both similarities and dissimilarities matter: Extending the concept of relational demography. Human Relations, 55, 899-929.

Turban, D. B. \& Jones, A. P. (1988). Supervisor-subordinate similarity: Types, effects, and mechanisms. Journal of Applied Psychology, 73, 228-234.

Van den Bos, K., Lind, E. A., \& Wilke, H. A. M. (2001). The psychology of procedural and distributive justice viewed from the perspective of fairness heuristic theory. In R. Cropanzano (Ed.), Justice in the workplace: From theory to practice (pp. 49-64). Mahwah, NJ: Lawrence Earlbaum.

Van den Bos, K., Wilke, H. A. M., \& Lind, E. A. (1998). When do we need procedural fairness? The role of trust in authority. Journal of Personality and Social Psychology, 75, 1449-1458.

Varma, A. \& Stroh, L. K. (2001). The impact of same-sex LMX dyads on performance evaluations. Human Resource Management, 40, 309-320.

Vecchio, R. P. (1993). The impact of differences in subordinate and supervisor age on attitudes and performance. Psychology and Aging, 8, 112-119. 
Venkataramani, V., Green, S. G., \& Schleicher, D. J. (2010). Well-connected leaders: The impact of leaders' social network ties on LMX and members' work attitudes. Journal of Applied Psychology, 95, 1071-1084.

Wakabayashi, M., Graen, G. B., Graen, M. R., \& Graen, M. G. (1988). Japanese management progress: Mobility into middle management. Journal of Applied Psychology, 73, 217-227.

Wang, H., Law, K. S., Hackett, R., Wang, D. X., \& Chen, Z. X. (2005). Leadermember exchange as a mediator of the relationship between transformational leadership and followers' performance and organizational citizenship behavior. Academy of Management Journal, 48, 420-432.

Wayne, S. J. \& Green, S. A. (1993). The effects of leader-member exchange on employee citizenship and impression management behavior. Human Relations, 46, 1431-1440.

Wayne, S. J., Shore, L. M., Bommer, W. H., \& Tetrick, L. E. (2002). The role of fair treatment and rewards in perceptions of organizational support and leadermember exchange. Journal of Applied Psychology, 87, 590-598.

Wayne, S. J., Shore, L. M., \& Liden, R. C. (1997). Perceived organizational support and leader-member exchange: A social exchange perspective. Academy of Management Journal, 40, 82-111. 
Wedell, D. H., Parducci, A., \& Gieselman, R. E. (1987). A formal analysis of ratings of physical attractiveness: Successive contrast and simultaneous assimilation. Journal of Experimental Social Psychology, 23, 230-249.

Wesley, D. \& Barczak, B. (2010). Innovation and marketing in the video game industry: Avoiding the performance trap. Burlington, VT: Ashgate Publishing.

Wessolowski, M. A. \& Mossholder, K. W. (1997). Relational demography in supervisor-subordinate dyads: Impact on subordinate job satisfaction, burnout, and perceived procedural justice. Journal of Organizational Behavior, 18, $351-362$.

Yan, A. \& Gray, B. (1994). Bargaining power, management control, and performance in United States-China joint ventures: A comparative case study. Academy of Management Journal, 37, 1478-1517.

Zohar, D. (2000). A group-level model of safety climate: Testing the effect of group climate on microaccidents in manufacturing jobs. Journal of Applied Psychology, 85, 587-596. 


\section{Appendix A: Measures}

Demographic variables

Participants were asked for the following demographic information:

1) Their own age

2) Their own gender

3) How long they had been working for the organization

4) How long they had been in their particular jobs

5) How long they had been working with their immediate supervisor

6) Their supervisor's age

7) Their supervisor's gender

8) How many times the supervisor had delivered performance feedback to them in the past

9) Their total years of education

10) Their monthly income (in Yuan, the Chinese currency)

Time 1 Variables

Leader-member Exchange (LMX)

Participants received the following instruction: "In the questions that follow, think about your relationship with your supervisor in general. Please indicate your level of agreement or disagreement by circling one of the seven alternatives next to each statement:" (response scale: 1 = "strongly disagree", 2 = "moderately disagree", 3 = "slightly disagree", 4 = "neutral", 5 = "slightly agree", $6=$ "moderately agree", 7 = "strongly agree").

1) Regardless of how much power he/she has built into his/her position, my supervisor would be personally inclined to use his/her power to help me solve problems in my work.

2) I can count on my supervisor to "bail me out", even at his or her own expense, when I really need it.

3) My supervisor understands my problems and needs.

4) My supervisor recognizes my potential. 
5) My supervisor has enough confidence in me that he/she would defend and justify my decisions if I were not present to do so.

6) I usually know where I stand with my supervisor.

7) I usually know how satisfied my supervisor is with me.

8) I would characterize the working relationship I have with my supervisor as extremely effective.

Perceived Informational Justice

Participants received the following instruction: "In the questions that follow, think about the content and substance of the most recent feedback and evaluation you received. Please indicate your level of agreement or disagreement by circling one of the seven alternatives next to each statement:" (response scale: $1=$ "strongly disagree", 2 = "moderately disagree", 3 = "slightly disagree", $4=$ "neutral", 5 = "slightly agree", 6 = "moderately agree", 7 = "strongly agree").

1) My supervisor was candid in his communications with me during the feedback event.

2) My supervisor explained the feedback thoroughly.

3) My supervisor's explanations were reasonable regarding the feedback event.

4) My supervisor communicated the details of the feedback to me in a timely manner.

5) My supervisor tailored his communications to my specific needs during the feedback event.

Perceived Interpersonal Justice

Participants received the following instruction: "In the questions that follow, think about the feedback giver's demeanor and behavior during your most recent feedback event. Please indicate your level of agreement or disagreement by circling one of the seven alternatives next to each statement:" (response scale: $1=$ "strongly disagree", 2 = "moderately disagree", 3 = "slightly disagree", 4 = "neutral", 5 = "slightly agree", 6 = "moderately agree", 7 = "strongly agree").

1) My supervisor treated me in a polite manner during the feedback event.

2) My supervisor treated me with dignity during the feedback event.

3) My supervisor treated me with respect during the feedback event. 
4) My supervisor refrained from making improper remarks or comments during the feedback event.

\section{Time 2 Variables}

\section{Employee Feedback Satisfaction}

Participants received the following instruction: "The following are more general questions about your perception of your most recent feedback event. Think specifically about how the feedback has made you feel. Please indicate your level of agreement or disagreement by circling one of the seven alternatives next to each statement:" (response scale: 1 = "strongly disagree", 2 = "moderately disagree", 3 = "slightly disagree", 4 = "neutral", 5 = "slightly agree", $6=$ "moderately agree", 7 = "strongly agree").

1) Based on what I contribute to my company, I am not satisfied with the feedback (reverse scored).

2) Considering the skills and the effort I put into my work, I am very satisfied with the feedback.

3) In general, the feedback measured up to what I expected.

4) The feedback was what I expected.

5) I am satisfied with my most recent performance appraisal.

6) Based on what I contribute to my company, I was fairly and accurately appraised during the feedback event.

7) Compared to others, I was evaluated fairly and accurately during the feedback event.

8) My performance was fairly and accurately evaluated during the feedback event.

9) I consider my most recent performance appraisal to be fair and accurate.

10) I understand why my supervisor evaluated me as he or she did during the feedback event.

11) I think evaluations are generally handled fairly in my organization.

12) I am satisfied with the manner in which I was evaluated by my supervisor during the feedback event.

Employee Perceptions of Feedback Utility 
Participants received the following instruction: "The following are more general questions about your perception of your most recent feedback event. Think specifically about how the feedback has made you feel. Please indicate your level of agreement or disagreement by circling one of the seven alternatives next to each statement:" (response scale: 1 = "strongly disagree", 2 = "moderately disagree", 3 = "slightly disagree", 4 = "neutral", 5 = "slightly agree", $6=$ "moderately agree", 7 = "strongly agree").

1) The feedback I was given was useful for my development as an employee.

2) Most of the material in the feedback seemed relevant.

3) The time I spent receiving the feedback was worthwhile.

4) I will be able to apply to my job what I learned from the feedback.

5) I will have opportunities to practice the skills emphasized in the feedback in my job.

Employee Work Motivation following the Feedback Event

Participants received the following instruction: "For the following questions, think about the impact this feedback had on you. The feedback might have affected your attitudes or behaviors in a positive or negative way... or it might not have had much impact at all. In the questions that follow, don't worry about things like your "absolute" level of motivation; rather, focus on the impact of the feedback (e.g., whether it raised, lowered, or didn't affect your motivation immediately following the feedback event). Please indicate the impact that the feedback had on the following by circling one of the seven alternatives next to each statement:" (response scale: 1 = "very negative impact", 2 = "moderately negative impact", 3 = "slightly negative impact", 4 = "neutral, very little impact", 5 = "slightly positive impact", $6=$ "moderately positive impact", $7=$ "very positive impact").

1) My desire to improve my performance.

2) My interest in developing as an employee.

3) My motivation to do a good job.

4) My desire to work hard in this position. 
DIRECTIONAL AGE DIFFERENCES IN CHINA

158

Table 1.

Inter-correlations among study variables

\begin{tabular}{|c|c|c|c|c|c|c|c|c|}
\hline & M & SD & 1 & 2 & 3 & 4 & 5 & 6 \\
\hline 1. Dir Age Diff & -4.12 & 8.11 & - & & & & & \\
\hline 2. Gend Cong & 1.80 & 0.40 & .00 & - & & & & \\
\hline 3. Dyad Tenure & 4.13 & 3.17 & .22 & .01 & - & & & \\
\hline 4. Valence & 3.79 & 0.75 & -.09 & -.02 & -.02 & $(.93)$ & & \\
\hline 5. LMX & 5.18 & 1.06 & -.07 & -.06 & .03 & $.38 * *$ & $(.87)$ & \\
\hline 6. Info Just & 5.51 & 1.18 & .02 & -.06 & .03 & $.50 * *$ & $.61 * *$ & $(.90)$ \\
\hline 7. Inter Just & 5.58 & 1.09 & -.06 & $-.11 *$ & .04 & $.39 * *$ & $.63 * *$ & $.65 * *$ \\
\hline 8. Satisfaction & 5.24 & 1.21 & $-.15 * *$ & -.09 & -.02 & $.55 * *$ & $.44 * *$ & $.47 * *$ \\
\hline 9. Utility perc. & 5.46 & 1.42 & $-.22 * *$ & -.08 & -.09 & $.43 * *$ & $.33 * *$ & $.35 * *$ \\
\hline 10. Motivation & 5.55 & 1.25 & $-.13 *$ & -.07 & -.02 & $.41 * *$ & $.32 * *$ & $.34 * *$ \\
\hline 11. Ee Age & 34.98 & 7.24 & $.79 * *$ & .01 & $.40 * *$ & -.05 & .01 & .07 \\
\hline 12. Sup Age & 39.10 & 5.00 & $-.47 * *$ & .01 & $.22 * *$ & .07 & $.14 * *$ & .07 \\
\hline
\end{tabular}

\begin{tabular}{l|ll|llllll}
\multicolumn{1}{l}{} & M & SD & 7 & 8 & 9 & 10 & 11 & 12 \\
\hline 1. Dir Age Diff & -4.12 & 8.11 & & & & & & \\
2. Gend Cong & 1.80 & 0.40 & & & & & \\
3. Dyad Tenure & 4.13 & 3.17 & & & & & \\
4. Valence & 3.79 & 0.75 & & & & & \\
5. LMX & 5.18 & 1.06 & & & & & \\
6. Info Just & 5.51 & 1.18 & & & & & \\
7. Inter Just & 5.58 & 1.09 & $(.85)$ & & & & \\
8. Satisfaction & 5.24 & 1.21 & $.46^{* *}$ & $(.96)$ & & & \\
9. Utility perc. & 5.46 & 1.42 & $.37^{* *}$ & $.80^{* *}$ & $(.97)$ & & \\
10. Motivation & 5.55 & 1.25 & $.36^{* *}$ & $.74^{* *}$ & $.73^{* *}$ & $(.96)$ & & \\
11. Ee Age & 34.98 & 7.24 & .00 & $-.12^{*}$ & $-.19^{* *}$ & $-.12^{*}$ & - & \\
12. Sup Age & 39.10 & 5.00 & .09 & .06 & .09 & .04 & $.16^{* *}$ & - \\
\hline
\end{tabular}

Note: * $p<.05$, ** $p<.01$

Positive Dir. Age Diff = younger supervisor

Gend Cong: $0=$ diff gender, 1 = same gender 
DIRECTIONAL AGE DIFFERENCES IN CHINA

Table 2.

SEM Path Analysis Coefficients

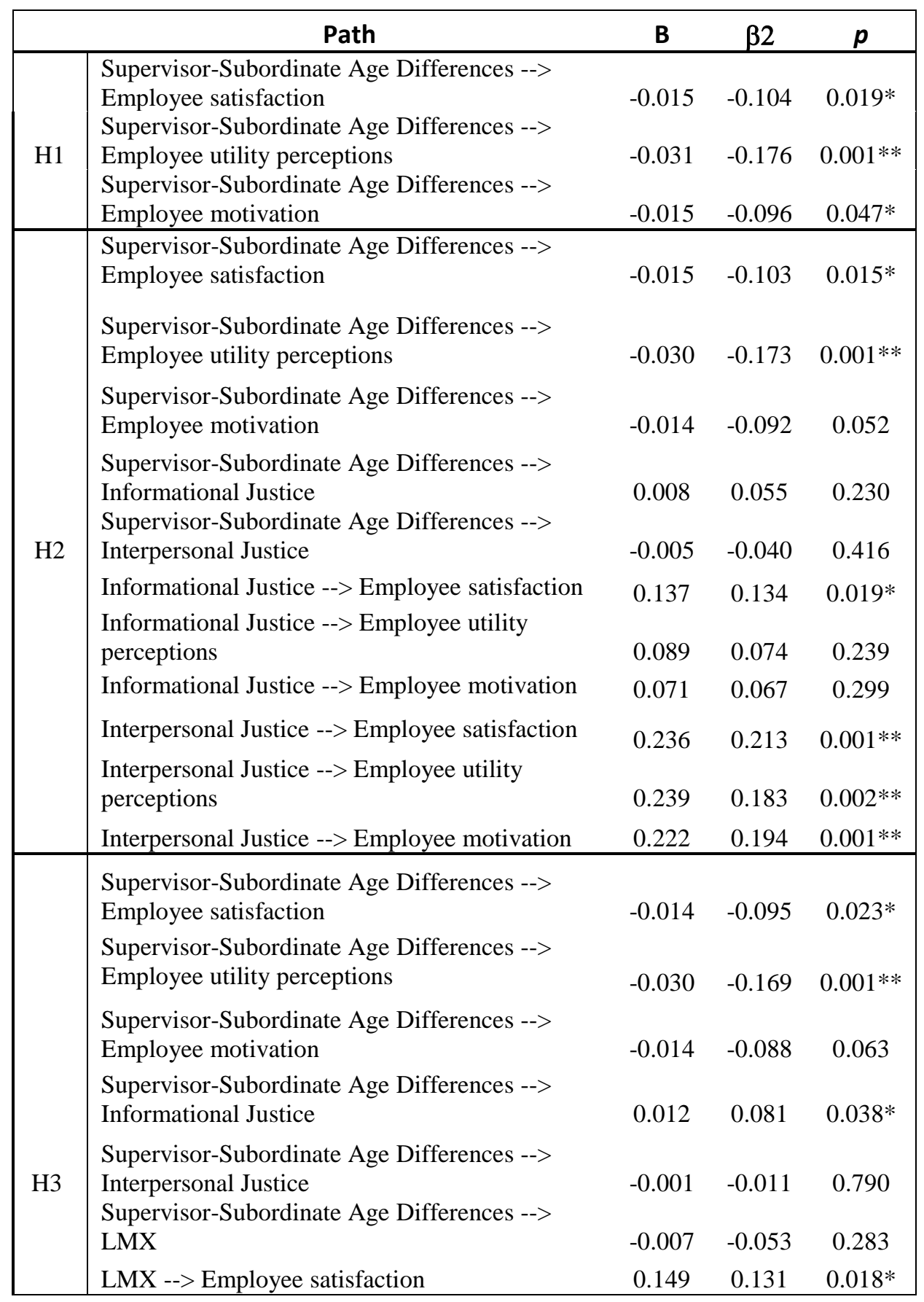


DIRECTIONAL AGE DIFFERENCES IN CHINA

\begin{tabular}{|c|c|c|c|c|}
\hline & Path & B & $\beta 2$ & $p$ \\
\hline \multirow{11}{*}{$\mathrm{H} 3$} & LMX --> Employee utility perceptions & 0.100 & 0.075 & 0.220 \\
\hline & LMX --> Employee motivation & 0.079 & 0.067 & 0.283 \\
\hline & LMX --> Informational Justice & 0.548 & 0.492 & $0.001 * *$ \\
\hline & LMX --> Interpersonal Justice & 0.567 & 0.553 & $0.001 * *$ \\
\hline & Informational Justice --> Employee satisfaction & 0.093 & 0.091 & 0.129 \\
\hline & Informational Justice --> Employee utility & & & \\
\hline & perceptions & 0.059 & 0.049 & $\begin{array}{r}0.456 \\
0.510\end{array}$ \\
\hline & Informational Justice --> Employee motivation & 0.047 & 0.045 & 0.510 \\
\hline & Interpersonal Justice --> Employee satisfaction & 0.181 & 0.164 & $0.004 * *$ \\
\hline & perceptiol & 0.202 & 0.155 & $0.015^{*}$ \\
\hline & Interpersonal Justice --> Employee motivation & 0.193 & 0.168 & $0.010^{*}$ \\
\hline \multirow{13}{*}{$\mathrm{H} 4$} & $\begin{array}{l}\text { Age Differences x LMX --> Employee } \\
\text { satisfaction }\end{array}$ & -0.011 & -0.010 & 0.815 \\
\hline & $\begin{array}{l}\text { Age Differences x LMX --> Employee utility } \\
\text { perceptions }\end{array}$ & 0.091 & 0.067 & 0.114 \\
\hline & $\begin{array}{l}\text { Age Differences x LMX --> Employee } \\
\text { motivation }\end{array}$ & 0.027 & 0.023 & 0.630 \\
\hline & Age Differences x LMX --> Informational & & & \\
\hline & Justice & 0.034 & 0.030 & 0.448 \\
\hline & $\begin{array}{l}\text { Age Differences x LMX --> Interpersonal } \\
\text { Justice }\end{array}$ & & & \\
\hline & Informational Justice --> Employee satisfaction & 0.094 & 0.092 & 0.126 \\
\hline & Informational Justice --> Employee utility & & & 0509 \\
\hline & & 0.052 & 0.043 & 0.509 \\
\hline & Informational Justice --> Employee motivation & 0.045 & 0.043 & 0.529 \\
\hline & Interpersonal Justice --> Employee satisfaction & 0.180 & 0.163 & $0.005^{* *}$ \\
\hline & $\begin{array}{l}\text { Interpersonal Justice --> Employee utility } \\
\text { perceptions }\end{array}$ & 0.209 & 0.160 & $0.011 *$ \\
\hline & Interpersonal Justice --> Employee motivation & 0.195 & 0.170 & $0.009^{* *}$ \\
\hline \multirow{7}{*}{ Full } & $\begin{array}{l}\text { Age Differences x LMX --> Employee } \\
\text { satisfaction }\end{array}$ & -0.011 & -0.010 & 0.815 \\
\hline & $\begin{array}{l}\text { Age Differences x LMX --> Employee utility } \\
\text { perceptions }\end{array}$ & 0.091 & 0.067 & 0.144 \\
\hline & $\begin{array}{l}\text { Age Differences x LMX --> Employee } \\
\text { motivation }\end{array}$ & 0.027 & 0.023 & 0.630 \\
\hline & Age Differences x LMX --> Informational & & & \\
\hline & Justice & 0.034 & 0.030 & 0.448 \\
\hline & $\begin{array}{l}\text { Age Differences x LMX --> Interpersonal } \\
\text { Justice }\end{array}$ & -0.031 & -0.030 & 0.465 \\
\hline & $\begin{array}{l}\text { Supervisor-Subordinate Age Differences --> } \\
\text { Employee satisfaction }\end{array}$ & -0.014 & -0.093 & $0.028^{*}$ \\
\hline
\end{tabular}


DIRECTIONAL AGE DIFFERENCES IN CHINA

161

\begin{tabular}{|c|c|c|c|c|}
\hline & Path & B & $\beta 2$ & $p$ \\
\hline \multirow{18}{*}{ Full } & $\begin{array}{l}\text { Supervisor-Subordinate Age Differences --> } \\
\text { Employee utility perceptions }\end{array}$ & -0.032 & -0.181 & $0.001 * *$ \\
\hline & $\begin{array}{l}\text { Supervisor-Subordinate Age Differences --> } \\
\text { Employee motivation }\end{array}$ & $=014$ & - 092 & 0063 \\
\hline & Supervisor-Subordinate Age Differences --> & & & \\
\hline & Informational Justice & 0.011 & 0.075 & 0.059 \\
\hline & Supervisor-Subordinate Age Differences --> & & & \\
\hline & $\begin{array}{l}\text { Interpersonal Justice } \\
\text { Supervisor-Subordinate Ag }\end{array}$ & -0.001 & -0.005 & 6 \\
\hline & LMX & -0.007 & -0.053 & 0.283 \\
\hline & LMX --> Employee satisfaction & 0.148 & 0.130 & $0.019 *$ \\
\hline & LMX --> Employee utility perceptions & 0.106 & 0.079 & 0.192 \\
\hline & LMX --> Employee motivation & 0.081 & 0.069 & 0.272 \\
\hline & LMX --> Informational Justice & 0.550 & 0.494 & $0.001 * *$ \\
\hline & LMX --> Interpersonal Justice & 0.565 & 0.550 & $0.001 * *$ \\
\hline & Informational Justice --> Employee satisfaction & 0.094 & 0.092 & 0.126 \\
\hline & $\begin{array}{l}\text { Informational Justice --> Employee utility } \\
\text { perceptions }\end{array}$ & 0.052 & 0.043 & 0.509 \\
\hline & Informational Justice --> Employee motivation & 0.045 & 0.043 & 0.529 \\
\hline & Interpersonal Justice --> Employee satisfaction & 0.180 & 0.163 & $0.005^{* *}$ \\
\hline & $\begin{array}{l}\text { Interpersonal Justice --> Employee utility } \\
\text { perceptions }\end{array}$ & 0.209 & 0.160 & $0.011^{*}$ \\
\hline & Interpersonal Justice --> Employee motivation & 0.195 & 0.170 & $0.009 * *$ \\
\hline
\end{tabular}

Note: $* p<.05, * * p<.01$. Supervisor-Subordinate Dir. Age Differences positive $=$ younger supervisor. 
Figure 1. Hypothesis 1 model

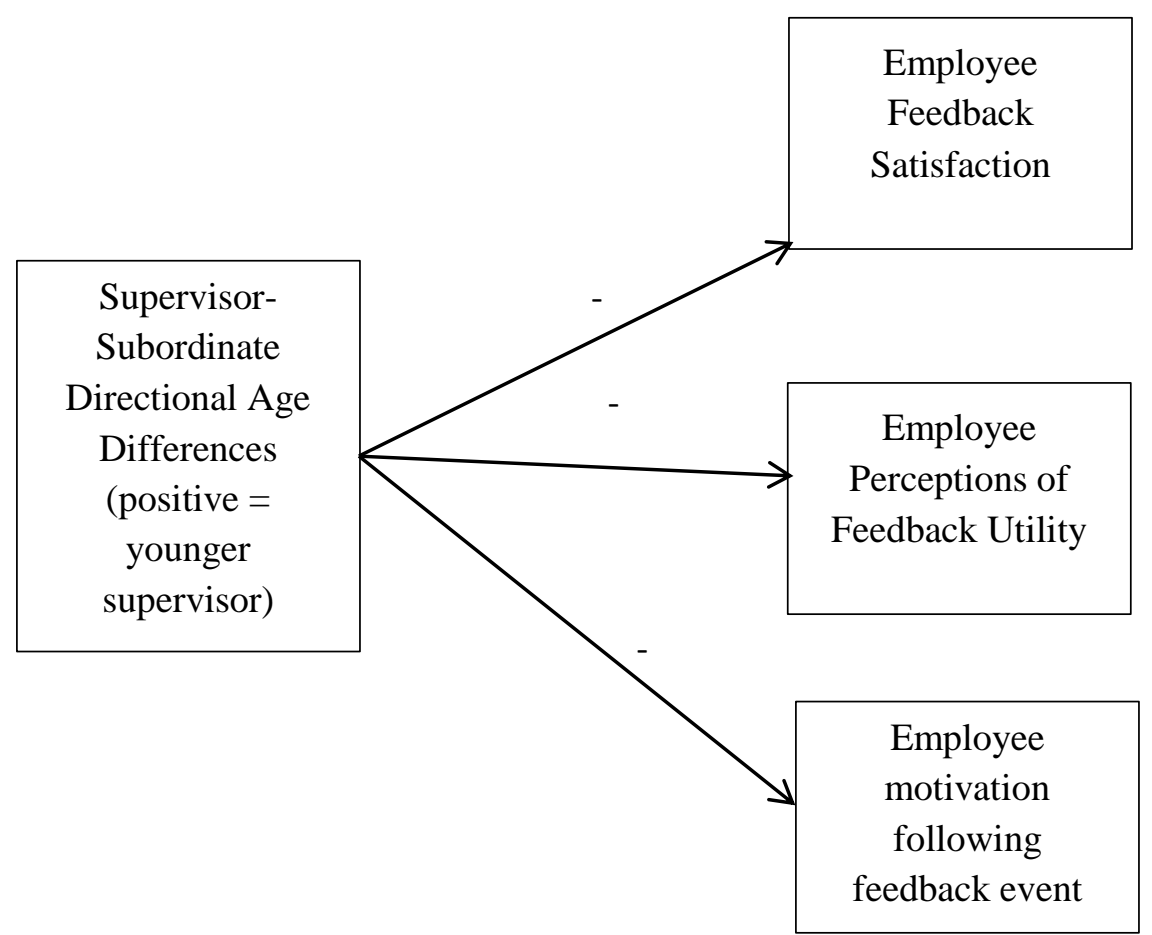


Figure 2. Hypothesis 2 model

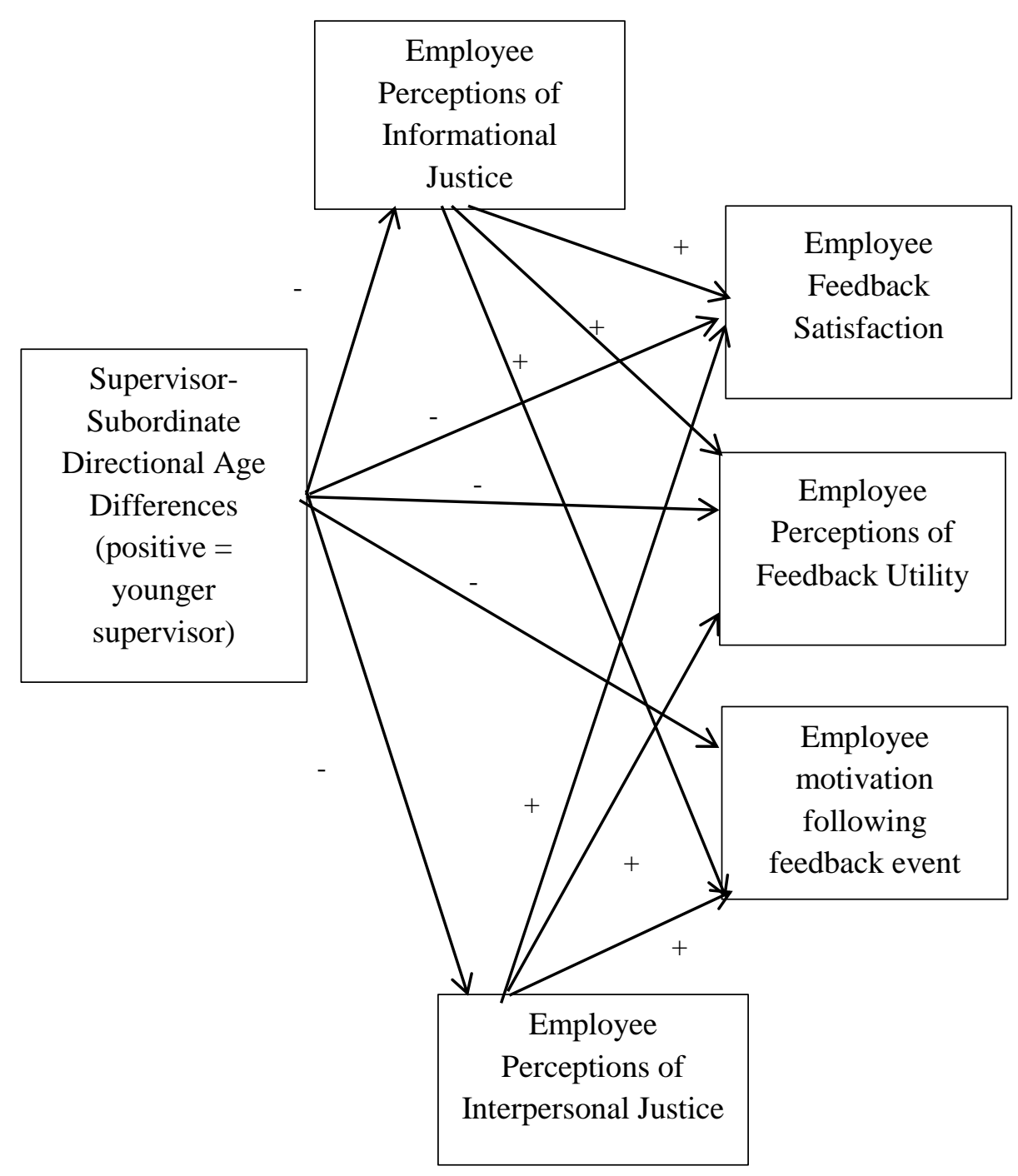


Figure 3. Hypothesis 3 model

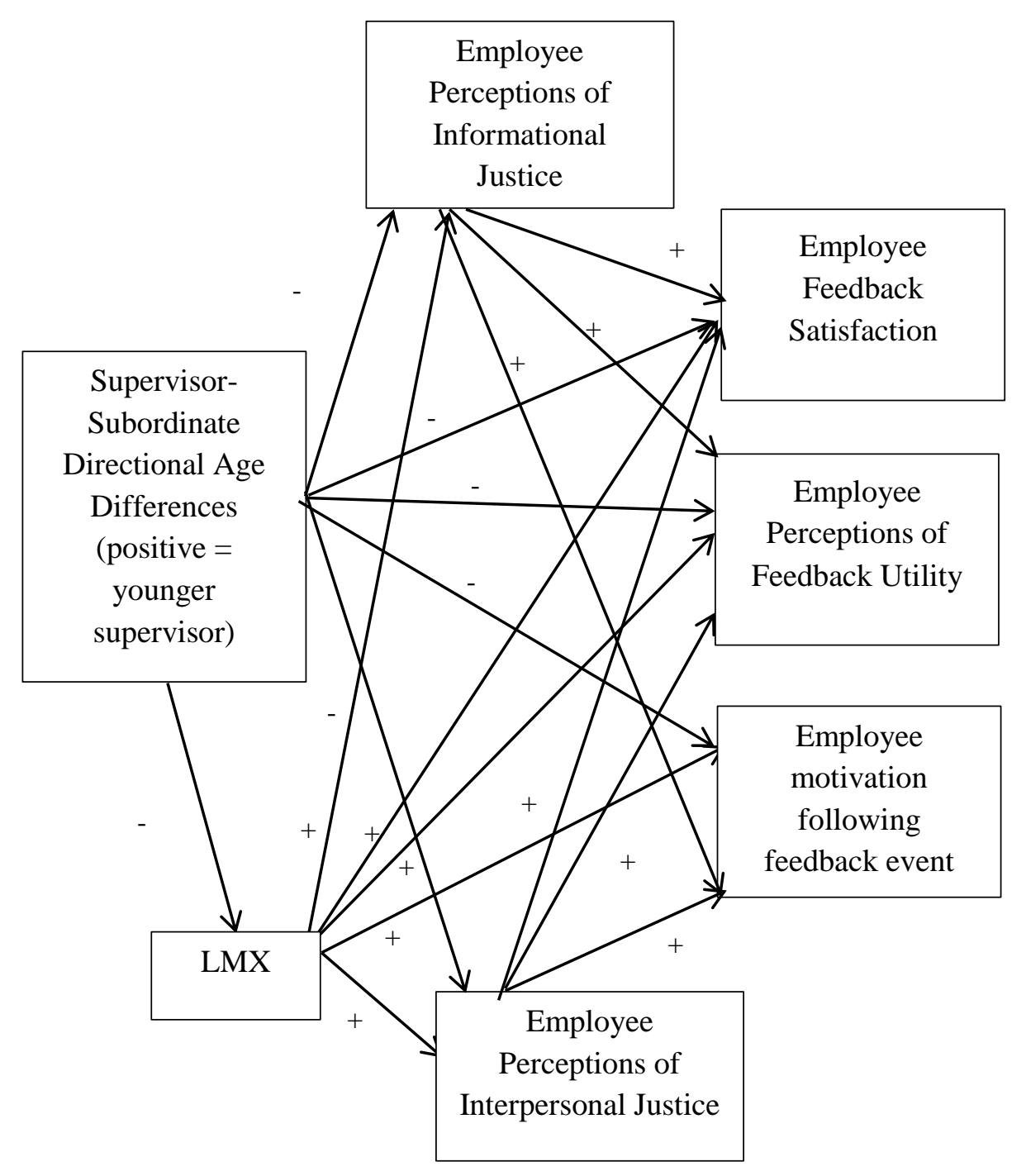


Figure 4. Hypothesis 4 model

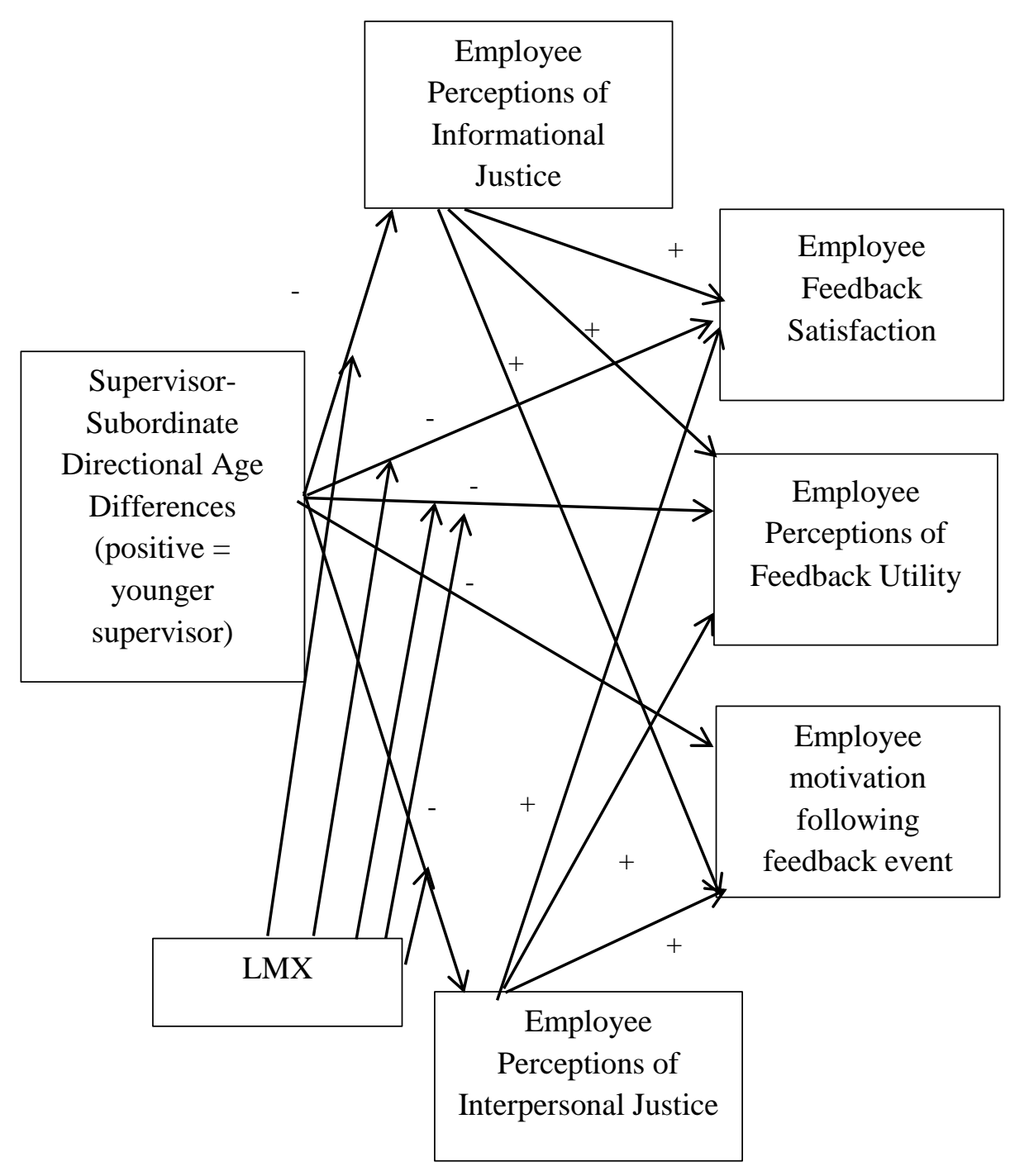


Figure 5. Hypothesis 1 unstandardized (and standardized) analysis path coefficients

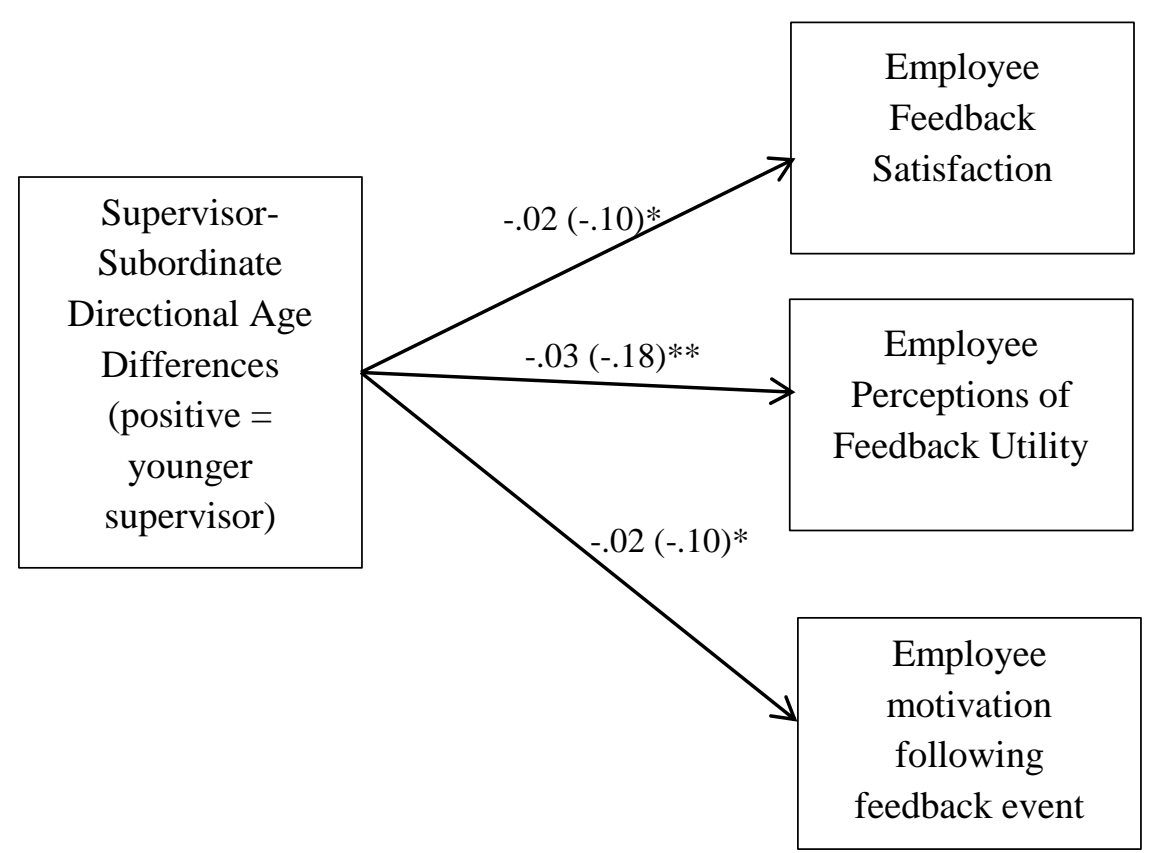

Note: $* p<.05, * * p<.01$ 
Figure 6. Hypothesis 2 unstandardized (and standardized) analysis path coefficients

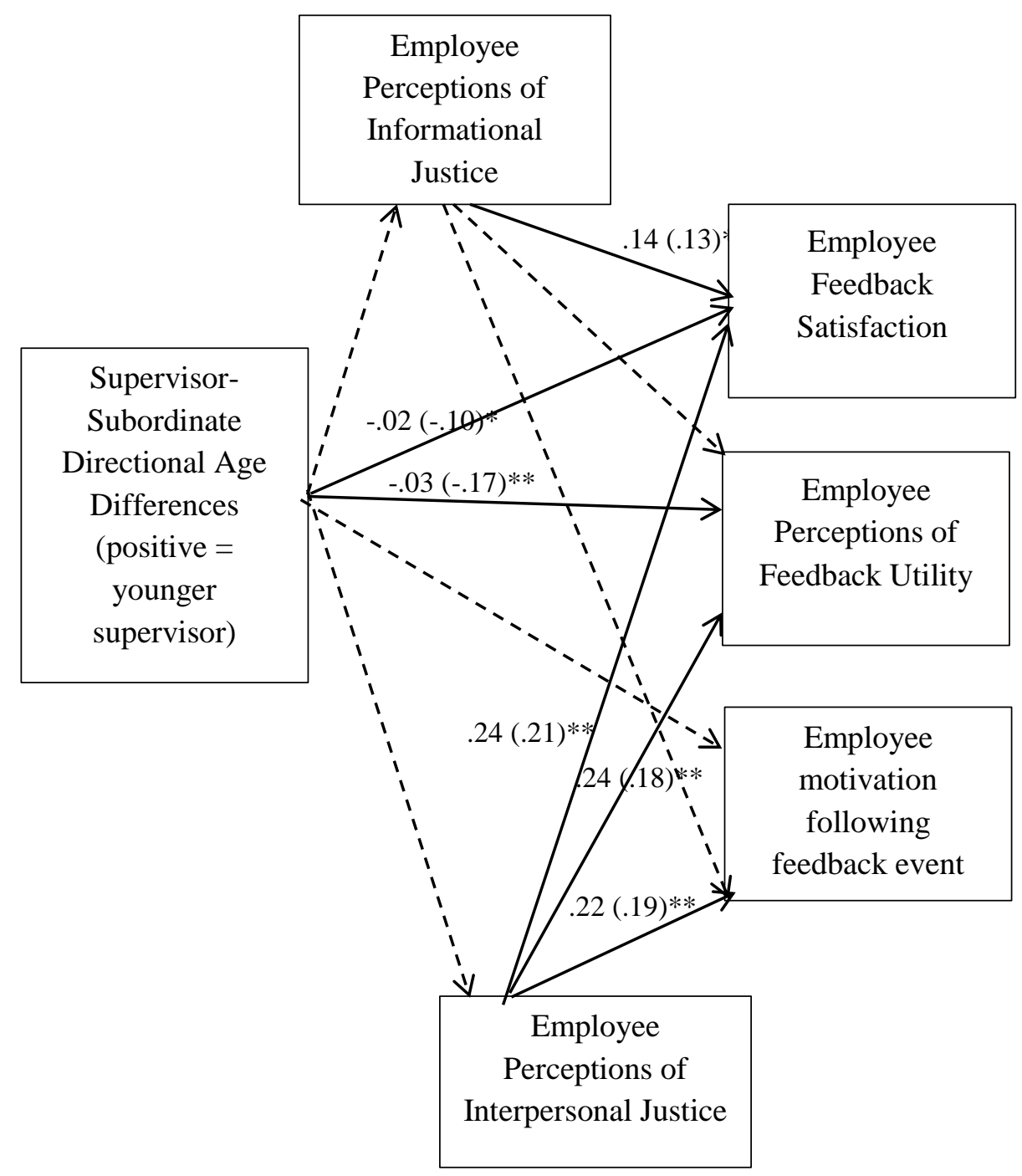

Note: $* p<.05, * * p<.01$

All ns paths are represented by dashed lines 
Figure 7. Hypothesis 3 unstandardized (and standardized) analysis path coefficients

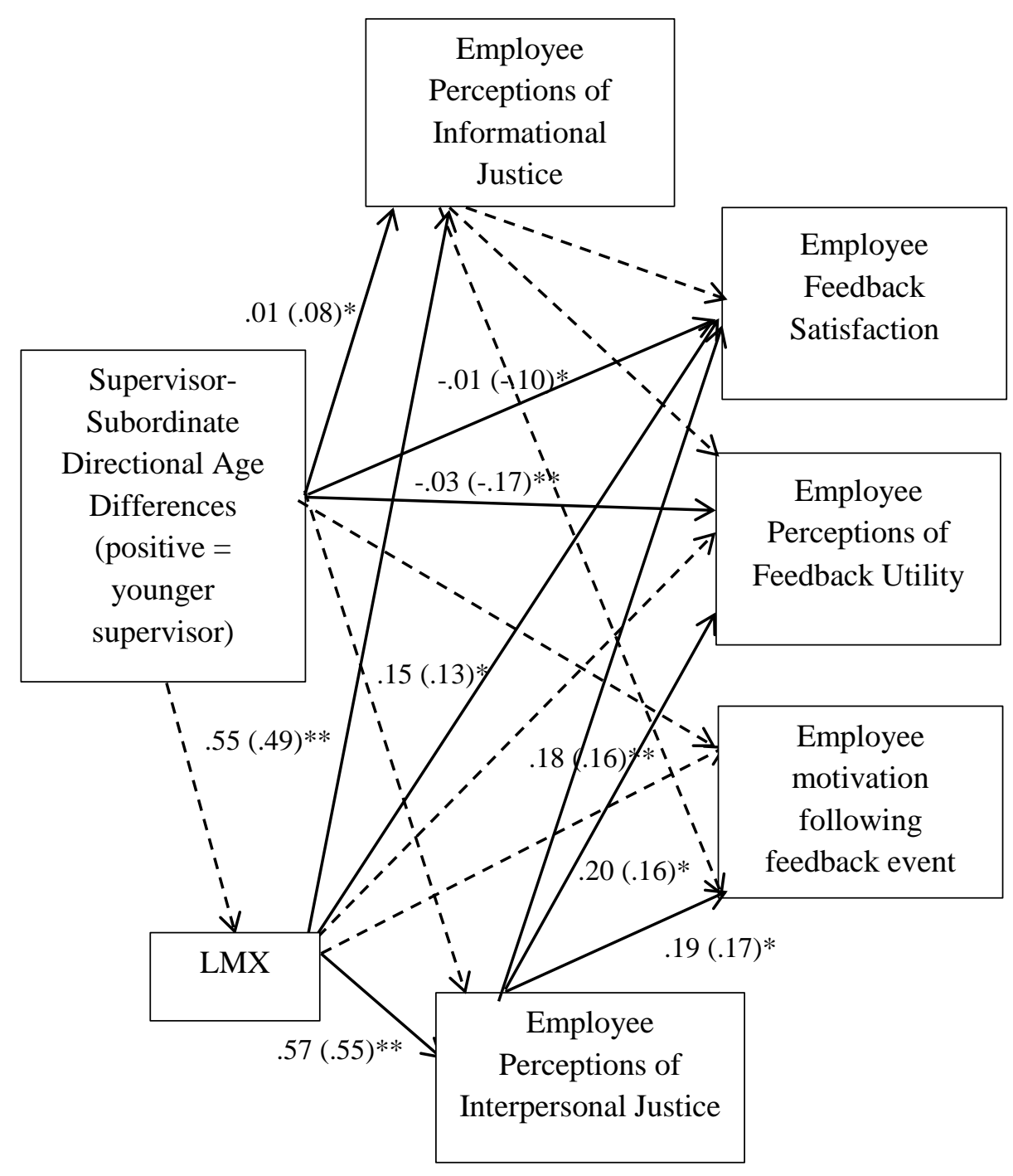

Note: $* p<.05, * * p<.01$

All ns paths are represented by dashed lines 
Figure 8. Hypothesis 4 unstandardized (and standardized) analysis path coefficients

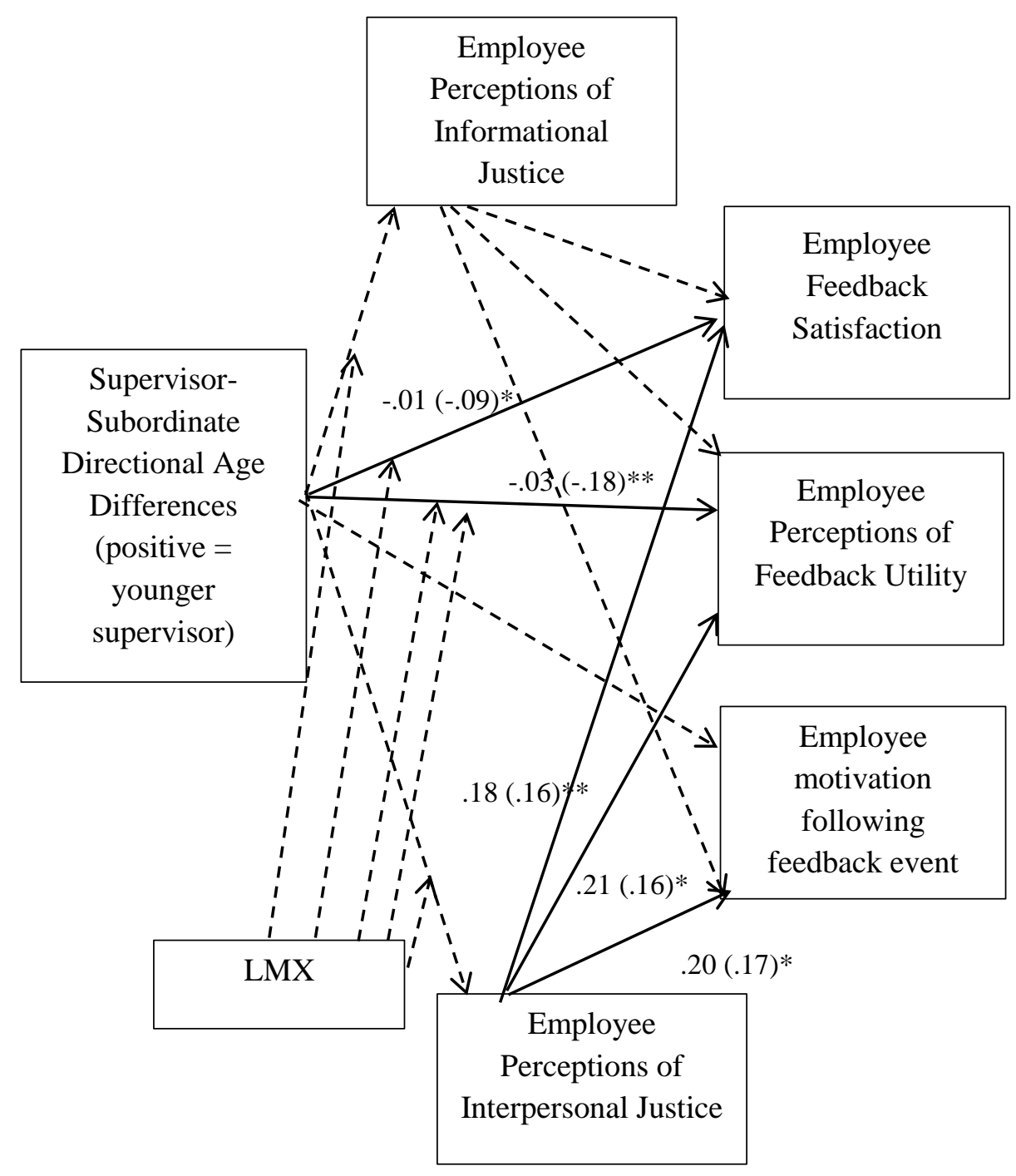

Note: $* p<.05, * * p<.01$

All ns paths are represented by dashed lines 
Figure 9. Full model unstandardized (and standardized) analysis path coefficients

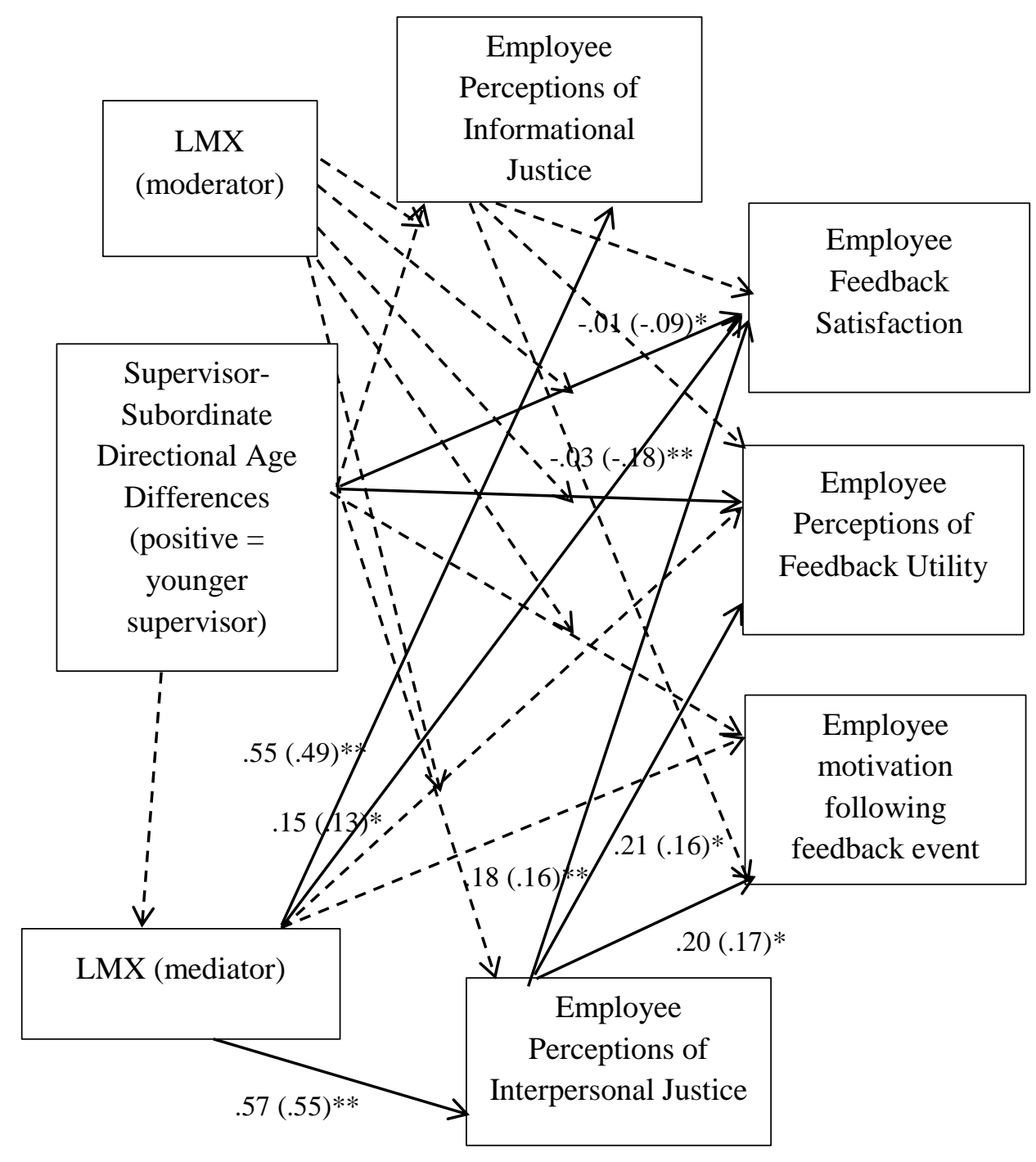

Note: $* p<.05, * * p<.01$

All ns paths are represented by dashed lines 
Figure 10. Feedback valence moderates the effect of supervisor-subordinate directional age differences on employee perceptions of LMX

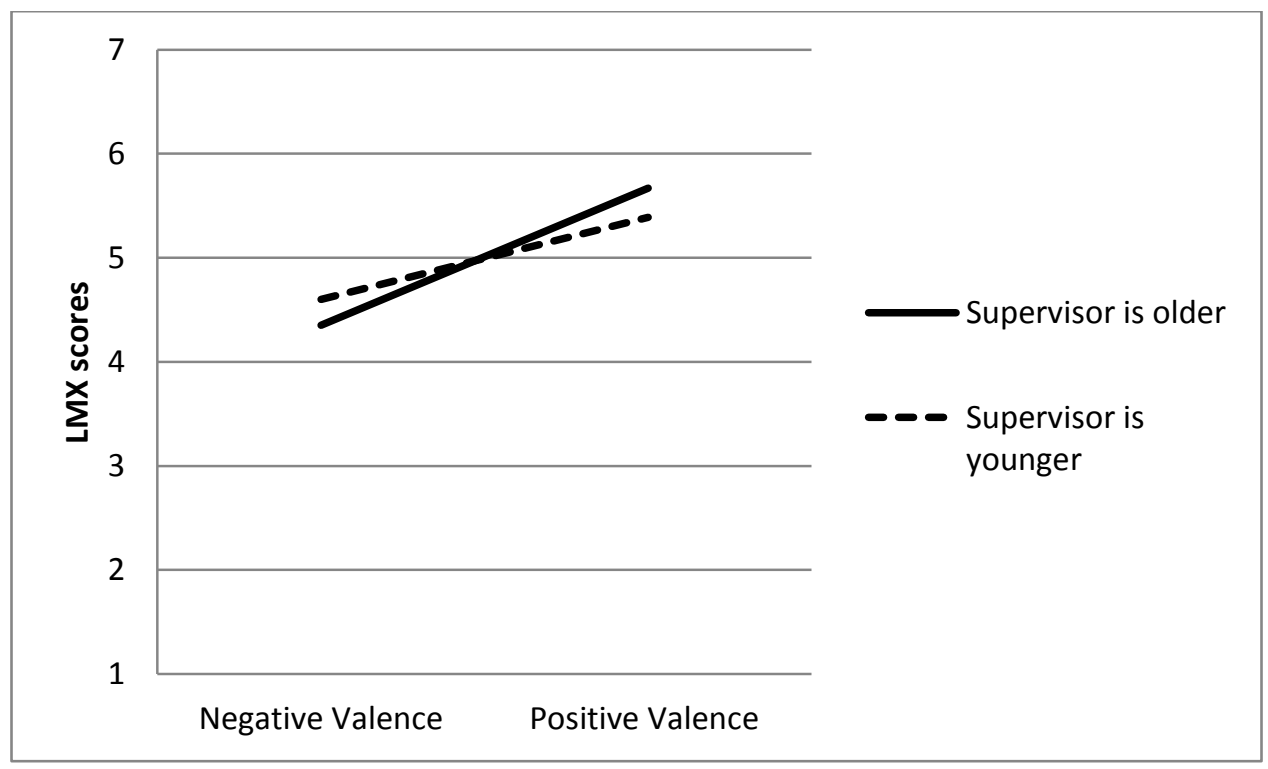


Figure 11. Feedback valence moderates the effect of supervisor-subordinate directional age differences on employee feedback satisfaction

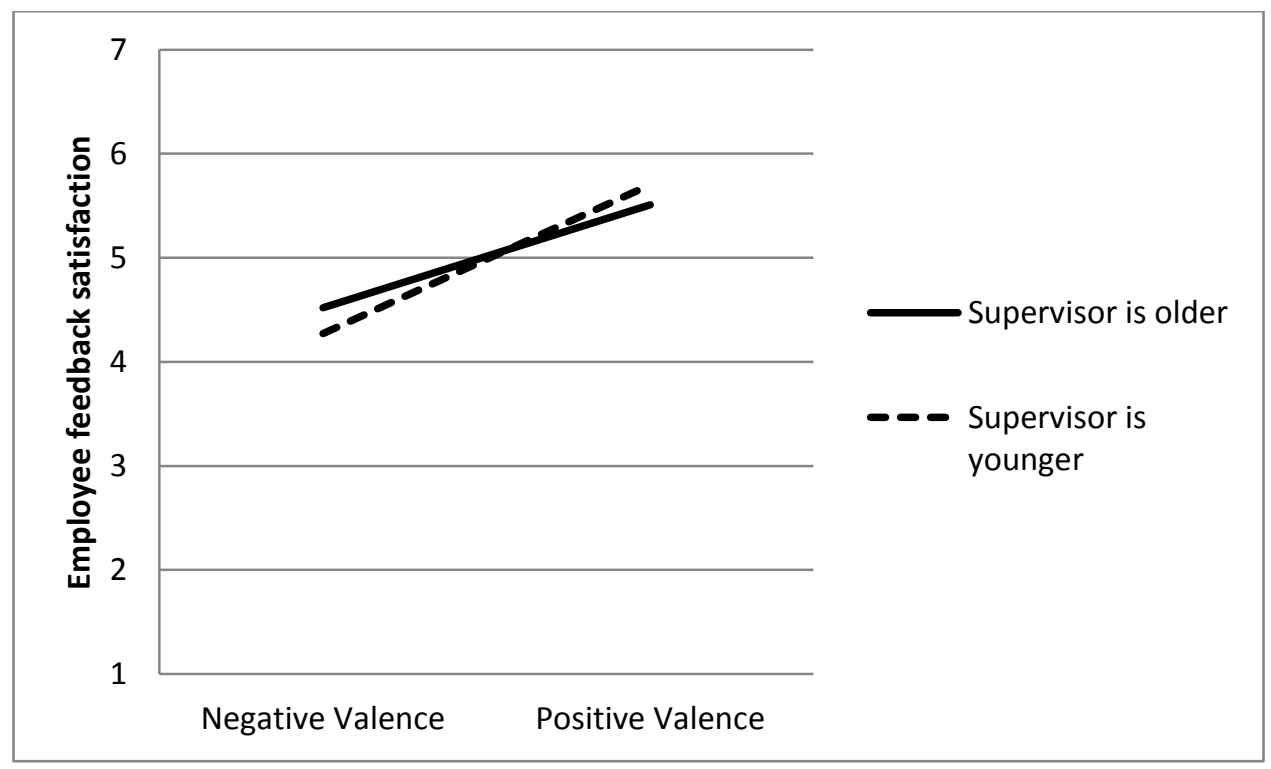


Figure 12. Feedback valence moderates the effect of supervisor-subordinate directional age differences on employee motivation following the feedback event

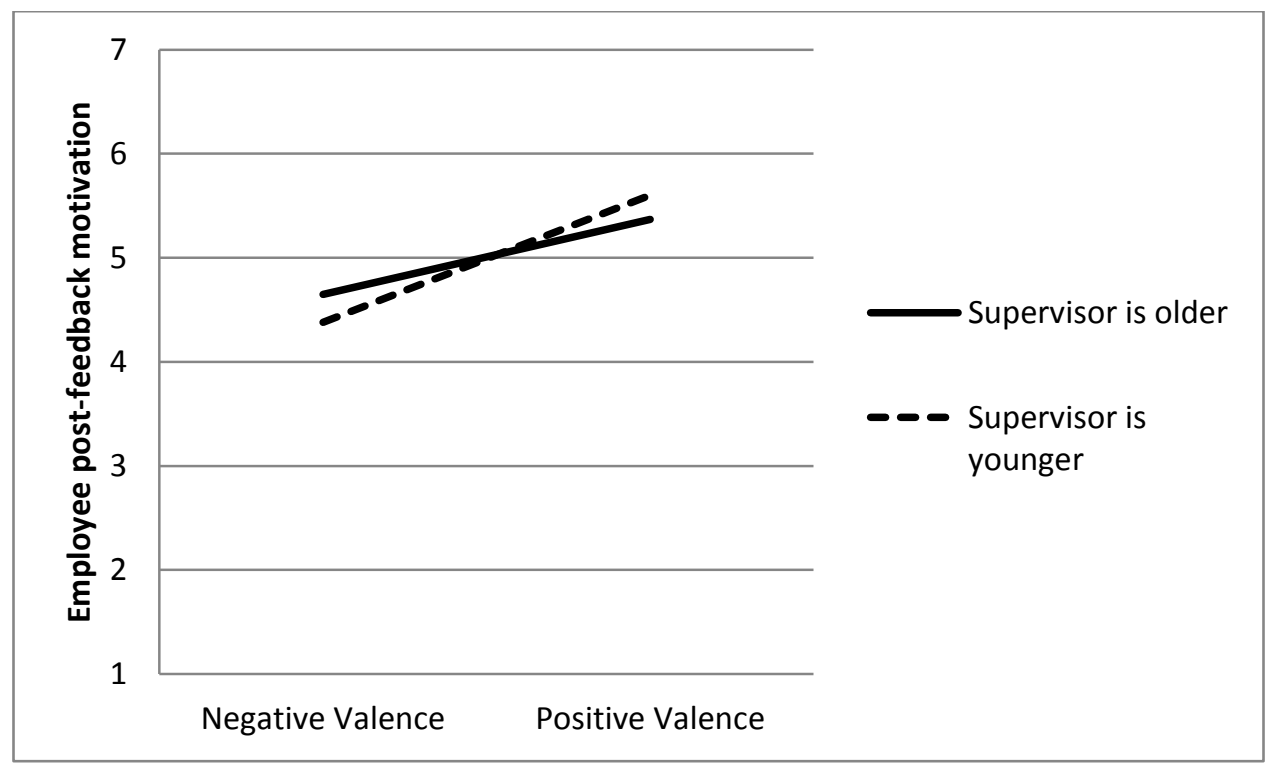

